Portland State University

PDXScholar

Fall 12-22-2015

\title{
Mechanisms of Adaptation in the Newly Invasive Species Brachypodium sylvaticum (Hudson) Beauv.
}

\author{
Gina Lola Marchini \\ Portland State University
}

Follow this and additional works at: https://pdxscholar.library.pdx.edu/open_access_etds

Part of the Ecology and Evolutionary Biology Commons, Genetics Commons, and the Plants

Commons

Let us know how access to this document benefits you.

\section{Recommended Citation}

Marchini, Gina Lola, "Mechanisms of Adaptation in the Newly Invasive Species Brachypodium sylvaticum (Hudson) Beauv." (2015). Dissertations and Theses. Paper 2640.

https://doi.org/10.15760/etd.2636

This Dissertation is brought to you for free and open access. It has been accepted for inclusion in Dissertations and Theses by an authorized administrator of PDXScholar. Please contact us if we can make this document more accessible: pdxscholar@pdx.edu. 
Mechanisms of Adaptation in the Newly Invasive Species Brachypodium sylvaticum (Hudson) Beauv.

by

Gina Lola Marchini

A dissertation submitted in the partial fulfillment of the requirements for the degree of

\author{
Doctor of Philosophy \\ in \\ Biology \\ Dissertation Committee: \\ Mitch Cruzan, Chair \\ Sarah Eppley \\ Suzanne Estes \\ Todd Rosenstiel \\ Wayne Wakeland
}

Portland State University

2015 
(C) 2015 Gina Lola Marchini 


\begin{abstract}
It is common knowledge that invasive species cause worldwide ecological and economic damage, and are nearly impossible to eradicate. However, upon introduction to a novel environment, alien species should be the underdogs: They are present in small numbers, possess low genetic diversity, and have not adapted to the climate and competitors present in the new habitat. So, how are alien species able to invade an environment occupied by native species that have already adapted to the local environment? To discover some answers to this apparent paradox I conducted four ecological genetic studies that utilized the invasive species Brachypodium sylvaticum (Hudson) Beauv. to determine mechanisms contributing to adaptation and success in the novel habitat.
\end{abstract}

The first study used simulations and experiments to test the hypothesis that genetic purging, the process where genetic load is reduced by selection against the recessive deleterious alleles expressed in the homozygous state, promotes invasive range expansion. I found that homozygous populations on B. sylvaticum's range periphery displayed lower inbreeding depression compared to heterozygous populations near introduction sites. Empirical tests with B. sylvaticum further demonstrate that purging of genetic load is a plausible scenario promoting range expansion during invasion.

Next, I explored how the interaction between population genetic diversity and the environment contributed to the establishment and spread of Brachypodium sylvaticum. I found that nitrogen application increases both final size and shoot biomass for $B$. sylvaticum individuals from source populations with low $H_{\mathrm{S}}$ levels to levels found in 
individuals from populations with high $H_{\mathrm{s}}$. A coefficient of relative competition intensity index $(R C I)$ displayed reduced effects of interspecific competition on B. sylvaticum biomass in high nitrogen plots. Results show that elevated nitrogen deposition is a factor that increases establishment of introduced species with historically small effective population sizes.

Thirdly, I investigated phenotypic differentiation during the establishment and range expansion of Brachypodium sylvaticum. Utilizing a novel approach, unique alleles were used to determine the genetic probability of contribution from native source regions to invasive regions. These probabilities were integrated into $Q_{\mathrm{ST}}-F_{\mathrm{ST}}$ comparisons to determine the influence of selection and genetic drift on twelve physiological and anatomical traits associated with drought stress. Phenotypic divergence greater than neutral expectations was found for five traits between native and invasive populations, indicating selective divergence. Results from this study show that the majority of divergence in B. sylvaticum occurred after introduction to the novel environment, but prior to invasive range expansion.

The final chapter of my dissertation investigates the adaptive role of genetic differentiation and plasticity for Brachypodium sylvaticum invasion. Plasticity was measured across treatments of contrasting water availability. Linear and nonlinear selection gradients determined the effect of directional and quadratic selection on plasticity and genetic differentiation. Invasive trait divergence was a consequence of post-introduction selection leading to genetic differentiation, as there were no plastic responses to contrasting water availability for any measured traits. Genetic divergence of 
invasive plants was not consistently in the direction indicated by selection, suggesting limitations of selection that may be a consequence of physical constraints and/or tradeoffs between growth and abiotic tolerance. Results suggest that selection, rather than plasticity, is driving phenotypic change in the invaded environment.

The combined volume of these studies contributes significantly to the field of invasion and plant biology by providing novel insights into the processes underlying range expansion, adaptation, and ultimately, evolution of introduced species. 


\section{Acknowledgements}

I would like to thank my advisor, Mitch Cruzan, for providing his knowledge, ideas, support, and encouragement over the years. I acknowledge and thank Sarah Eppley, Suzanne Estes, Todd Rosenstiel, and Wayne Wakeland for contributing their valuable time to serve on my committee.

I would like to state my appreciation for the opportunity to serve as a fellow in the NSF GK-12 Cascades to Coast program. Specifically to Cat DeRivera, Linda George, and Patrick Edwards, organizers of the program at Portland State University. I am grateful to all the awesome GK-12 fellows that I got to work and learn with throughout the years, including Nicole Alfafara, Sarah Kidd, Monica Mogilewsky, and Hannah Prather.

Thank you to my labmates, past and present, for your friendship and enthusiasm. There are many of you who worked in the lab, either as undergraduate researchers, graduate students, or postdoctoral researchers, and you provided me with excellent feedback on many projects. These labmates are Tanya Cheeke, Nena Cole, Monica Grasty, Brendan Kohrm, Susan Jaconis, Caitlin Lee, Trieste Musial, Caitlin Maraist, Alisa Ramakrishnan, David Rosenthal, Tina Schroyer, Laura Taylor, Pam Thompson, Ben Weinstien, Rachel Workman, and Michelle Williamson.

And of course, thank you to my friends and family. My mom and dad who have been my cheerleaders throughout graduate school. My siblings Laila, Alex, and Charlie. And thank you Casey, for being there for me always. 


\section{Table of Contents}

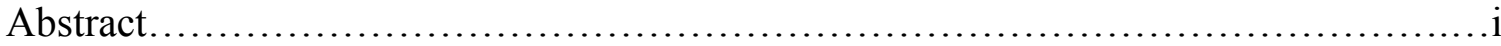

Acknowledgements.............................................................

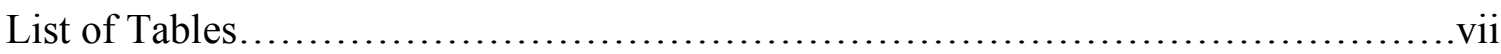

List of Figures............................................................. vii

\section{Chapter 1}

Introduction.

\section{Chapter 2}

Rapid Purging of Genetic Load in a Metapopulation and Consequences for

Range Expansion in an Invasive Plant...

\section{Chapter 3}

Ecological mitigation of inbreeding depression in a newly invasive

species

\section{Chapter 4}

Selective differentiation during the colonization and establishment of a newly invasive species....

\section{Chapter 5}

Functional Trait Divergence, Not Plasticity, Determines the Success of a Newly Invasive

Plant.

\section{Chapter 6}

Conclusions: Management Implications of Studies

References.

Appendices

\section{Appendix A}

Supplemental materials to Chapter 2: Rapid Purging of Genetic Load in a Metapopulation and Consequences for Range Expansion in an Invasive Plant.

\section{Appendix B}

Supplemental materials to Chapter 3: Ecological mitigation of inbreeding depression in a newly invasive species. 


\section{Appendix C}

Supplemental materials to Chapter 4: Selective differentiation during the colonization and establishment of a newly invasive species....................................157

\section{Appendix D}

Supplemental materials to Chapter 5: Functional Trait Divergence, Not Plasticity, Determines the Success of a Newly Invasive Plant..................................159 


\section{List of Tables}

Table 3.1 Location information, $H_{S}, H_{O}$, and $F_{I S}$ values for $B$. sylvaticum populations...57

Table 3.2 Statistical results presented as F-values..............................58

Table 4.1 Location, latitude and longitude, sample size $(\mathrm{n})$, gene diversity $\left(H_{\mathrm{S}}\right)$, and inbreeding coefficients $\left(F_{\mathrm{IS}}\right)$ for populations.

Table 4.2 Functional traits measured in B. sylvaticum and their units, significance, and expected behavior in drought.......................................... 87

Table 4.3 Variance components, $F_{\mathrm{ST}}, Q_{\mathrm{ST}}$, and $Q_{\mathrm{ST}}-F_{\mathrm{ST}}$ for populations across the

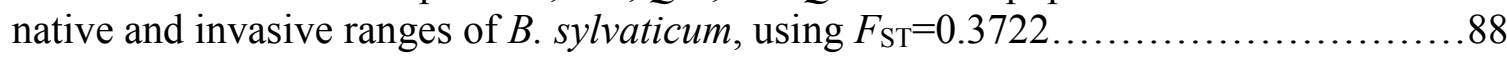

Table 4.4 Variance components, $F_{\mathrm{ST}}, Q_{\mathrm{ST}}$, and $Q_{\mathrm{ST}}-F_{\mathrm{ST}}$ for populations in the invasive range of $B$. sylvaticum, using $F_{\mathrm{ST}}=0.4609$

Table 5.1 P-values from mixed linear regressions finding the effects of range, abiotic environment, and treatment on 6 measured traits in Brachypodium sylvaticum.........118

Table 5.2 Directional $(\beta)$ and quadratic $(\gamma)$ selection for traits of invasive and native populations of Brachypodium sylvaticum.

Table 5.3 Directional $(\beta)$ selection gradients of plasticity for traits of invasive and native populations of Brachypodium sylvaticum. 


\section{List of Figures}

Figure 1.1 Oregon forests before and after Brachypodium sylvaticum invasion..........6

Figure 1.2 Brachypodium sylvaticum native population locations.....................7

Figure 1.3 Overarching hypothesis of mechanisms underlying Brachypodium sylvaticum's invasion.... .8

Figure 2.1 A qualitative model for the dynamics of range expansion under selection and purging of genetic load in an outbreeding invasive species

Figure 2.2 Effects of population size and number of loci on purging...................33

Figure 2.3 The effects of metapopulation structure, breeding system, and gene flow on changes in the frequencies of deleterious mutations.

Figure 2.4 Stages of range expansion after establishment of an outbreeding invasive species

Figure 2.5 The effects of levels of divergence among established populations $\left(F_{S T}\right)$ on the potential for purging and rapid range expansion

Figure 2.6 The relationship between population genetic diversity and plant biomass in a common garden

Figure 2.7 The fitness of selfed (spotted) and outcrossed (striped) progeny from populations that vary for genetic diversity

Figure 3.1: Environmentally dependent-inbreeding depression (EDID) and environmental mitigation of inbreeding depression (EMID) of individuals in nutrient-rich and nutrient-poor conditions.

Figure 3.2: Nitrogen $x H_{\mathrm{S}}$ effects on final size (height*shoot number) and Nitrogen $\mathrm{x} H_{\mathrm{S}}$ effects on shoot biomass of $B$. sylvaticum focal plants.

Figure 3.3: Relative competition index coefficient (RCI) values for each per high and low nitrogen treatment for each source population $H_{\mathrm{s}}$....

Figure 4.1 Probability of genetic contribution to invasive populations of $B$. sylvaticum from 21 populations from throughout the native range

Figure 4.2 Traits exhibiting significant differences at $p<0.05$ between invasive and native population using data unweighted by the probability 
of genetic contribution.

Figure 4.3 Traits exhibiting significant differences at $p<0.05$ between invasive and native population using data weighted by the probability of genetic contribution

Figure 4.4 Null distributions of $Q_{\mathrm{ST}}-F_{\mathrm{ST}}$ for five quantitative traits with significant values of $Q_{\mathrm{ST}}-F_{\mathrm{ST}}$ in $B$. sylvaticum.

Figure 5.1 Principal components biplot of variables associated with climate in populations of Brachypodium sylvaticum.

Figure 5.2. Trait means for individuals from the invasive and native range of Brachypodium sylvaticum.

Figure 5.3. Examples of Brachypodium sylvaticum leaves from invasive and native ranges

Figure 5.4. Environmental gradients and phenotypic expression for four morphological traits of Brachypodium sylvaticum

Figure 5.5. Significant negative directional selection $(\beta)$ for plasticity in Brachypodium sylvaticum ......................................................... 125

Figure 5.6. Quadratic selection $(Y)$ for in Brachypodium sylvaticum .................126 


\section{Chapter 1}

\section{Introduction}

In the Origin of Species, Charles Darwin wrote that because competition drives natural selection, we should "feel no surprise at the inhabitants of any one country [...] being beaten and supplanted by the naturalised productions from another land." Darwin's writings regarding the fragility of ecosystems had raised an important question: How are alien species able to invade an environment occupied by native species that are adapted to that same environment? Despite Darwin's note on the existence of introduced organisms, it wasn't until almost a century later, with the publication in 1958 of The Ecology of Invasions by Animals and Plants by Charles Elton that an author endeavored to describe the ecological impact of invasive species and their consequences for biodiversity. Invasive species became of interest to evolutionary biologists after works in The Genetics of Colonizing Species, edited by Herbert G. Baker and G. Ledyard Stebbins and published in 1965, hypothesized that local adaptation and selection play major roles in invasion. The Genetics of Colonizing Species is considered to have laid the groundwork for the many modern studies of invasion genetics that had emerged by the end of the $20^{\text {th }}$ century.

The emergence of invasion biology as a popular field of study corresponds to an increasing rate of introduction of species into novel environments. Although humans have transported plants and animals around the earth for thousands of years, the everincreasing movement of people and goods worldwide has provided more opportunities than ever for species introductions (Mooney and Cleland 2001; Hulme 2009; Richardson and Rejmánek 2011). Invasive species are defined as organisms that are non-native to 
their current environment, can survive without direct human involvement, and have spread from sites of initial establishment in the introduced range. Although a small percentage of introduced species become invasive, introductions have accumulated over time, and by 2004 it was estimated that there were over 50,000 invasive species worldwide (Pimentel et al. 2005).

The prevalence of invasive species introduces a paradox: upon introduction to a novel environment, loss of genetic diversity through genetic drift and inbreeding should result in extinction. Introduced populations are thought to possess small numbers, low genetic diversity $\left(H_{\mathrm{S}}\right)$, and low effective population sizes $\left(N_{\mathrm{e}}\right)$, factors that limit the evolutionary potential of introduced populations. Several studies have predicted that invasive success is dependent on adaptation and species response to natural selection (Sakai et al. 2001; Lee 2002; Lavergne and Molofsky 2007; Prentis et al. 2008; Matzek 2012).

A partial answer to this apparent paradox of invasion has been found in the prevalence of multiple introductions contributing to invasion. A meta-analysis by Dlugosch and Parker (2008) and their accompanying studies with the invasive St. John's Wort species Hypericum canariense found that multiple introductions tend to be the rule, rather than the exception, when examining plants that have become invasive. Multiple introductions raise the $N_{\mathrm{e}}$ and $H_{\mathrm{S}}$ of introduced populations, essentially increasing population's evolutionary potential.

My dissertation work utilized Brachypodium sylvaticum (Hudson) Beauv., a bunchgrass currently invading the Pacific Northwest USA to test predictions of adaptation in invasive species, while also investigating the underlying genetic 
mechanisms facilitating adaptation. Brachypodium sylvaticum is a diploid, selfcompatible, perennial C-3 bunchgrass (Kaye 2003; Ramakrishnan et al. 2010). It is thought that the United States Department of Agriculture first introduced B. sylvaticum to central Oregon, USA in the 1920s while testing for a productive range grass, although populations did not become invasive until the end of the $20^{\text {th }}$ century (Hull 1974; Ramakrishnan et al. 2010). Brachypodium sylvaticum invades the shady understory of the Pacific Northwest, transforming areas once characterized by swordfern (Polystichum munitum), Oregon grape (Mahonia aquifolium), snowberry (Symphoricarpos albus), and redwood sorrel (Oxalis oregano) into vast monocultures of slender false brome (Fig. 1.1).

A study by Rosenthal et al. (2008) confirmed that there were two separate introductions of B. sylvaticum; one into Eugene, Oregon, USA, and the second into Corvallis, Oregon, USA. These introductions consisted of individuals from populations across B. sylvaticum's native range (Fig. 1.2). These multiple introductions resulted in the populations of initial establishment having high $H_{\mathrm{S}}$ and $N_{\mathrm{e}}$ and led to the development of recombinant hybrid genotypes in the invasive region as individuals from populations across the native region crossed (Rosenthal et al. 2008).

This known background of $B$. sylvaticum's introduction history allowed me to develop an overarching hypothesis for my dissertation (Fig. 1.3). Brachypodium sylvaticum was introduced from multiple sources, allowing the formation of the invasive genotypes. However, after these individuals began to spread into Oregon, populations underwent secondary bottlenecks in the introduced range, resulting in a halted range expansion. After populations overcame these secondary bottlenecks, invasive $B$. sylvaticum began its full invasion. In my dissertation, I seek to discover what processes 
underlie B. sylvaticum's ability to overcome these secondary bottlenecks in the introduced range, allowing the species to become invasive. These findings can be applied to investigations of mechanisms underlying invasions of other introduced plants, or of plants expanding their range into novel territories.

- In Chapter 2 I use empirical studies and simulations to investigate the hypothesis that genetic purging, the process where genetic load is reduced by selection against the recessive deleterious alleles expressed in the homozygous state, promotes invasive range expansion.

- In Chapter 3 I use greenhouse experiments to discover how addition of nitrogen to soils influence the competitive performance of $B$. sylvaticum individuals from populations with varying levels of genetic diversity. I test for ecological mitigation of inbreeding depression (EMID), the potential for stress reductions to augment fitness in populations with limited gene diversity, as a factor facilitating invasion.

- In Chapter 4 I determine if genetic differentiation of individuals in the invasive range of $B$. sylvaticum is the result of genetic drift or natural selection, additionally investigating if differentiation has occurred during the primary establishment phase of invasion or during subsequent range expansion. I also describe a robust method for detection of selective processes after species range expansion or introduction to a novel environment.

- In Chapter 5 I investigate phenotypic plasticity as a factor contributing to invasion. The adaptive value of phenotypic plasticity and genetic differentiation in the invasive range is determined. 
My dissertation work significantly contributes to the field of invasion biology by uncovering mechanisms underlying the establishment success of introduced species, despite low population genetic diversity. In a greater context, results from these studies provide insight into how populations adapt to changing environments. 


\section{Figures}

Figure 1.1 The forest understory in central Oregon, USA before (top, A) and after (bottom, B) Brachypodium sylvaticum invasion.
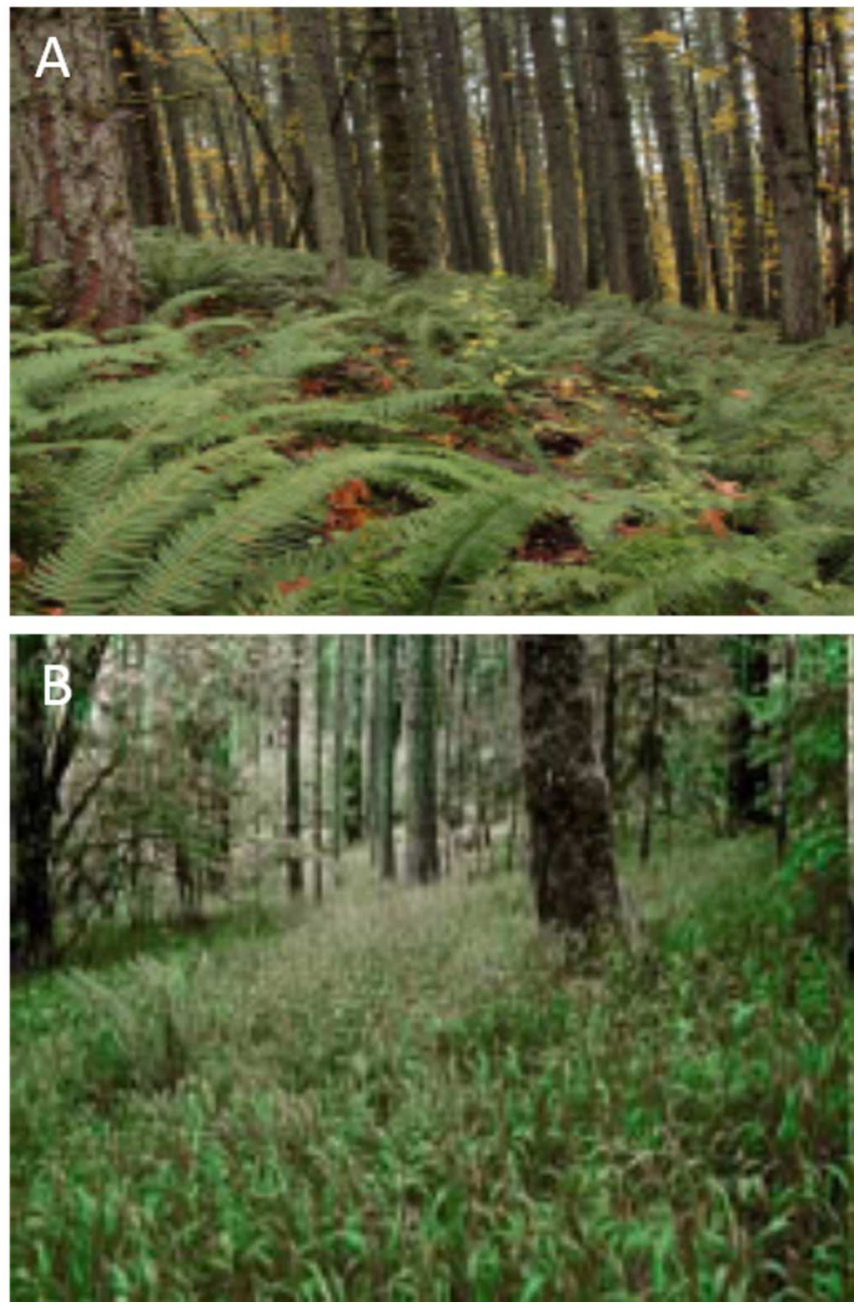
Figure 1.2 Locations of populations (black circles) in the native range of Brachypodium sylvaticum where individuals contributing to the original introduction are thought to have originated.

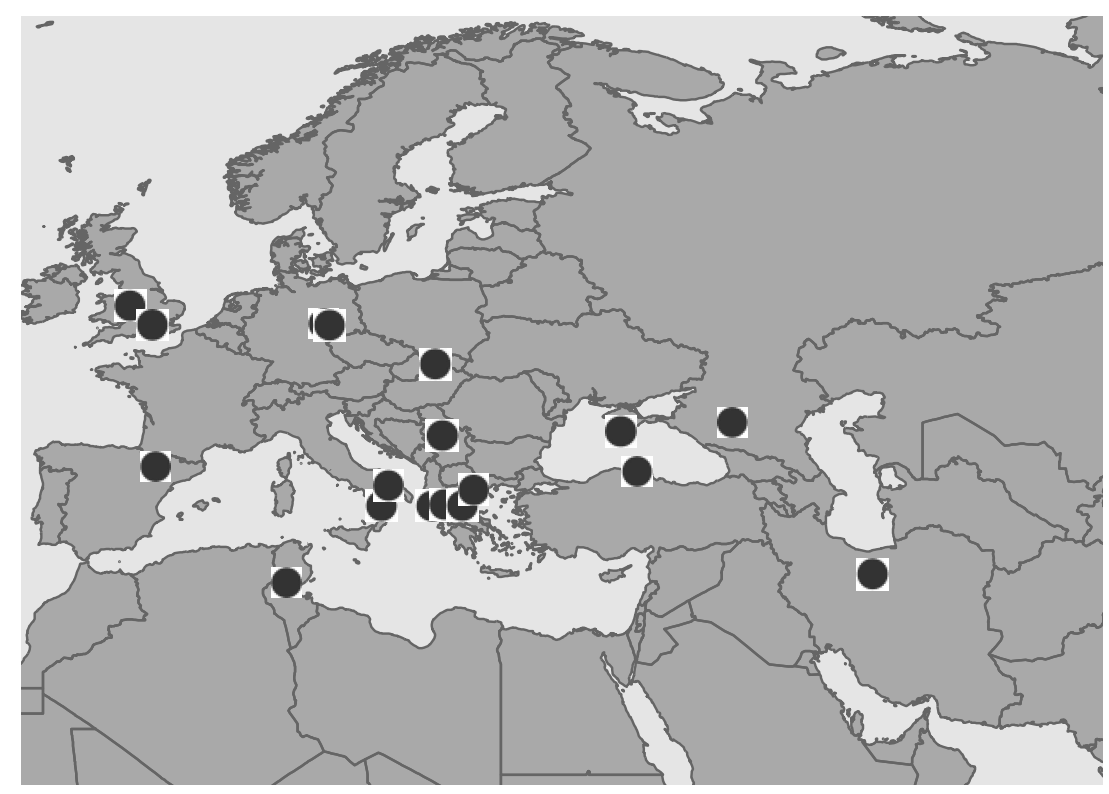


Figure 1.3 Overarching hypothesis of mechanisms underlying Brachypodium sylvaticum's invasion. Introduction from multiple source regions results in the development of an invasive genotype. After primary establishment, secondary bottlenecks occur as the species expands its range, slowing spread. After these bottlenecks are overcome, a full invasion begins.

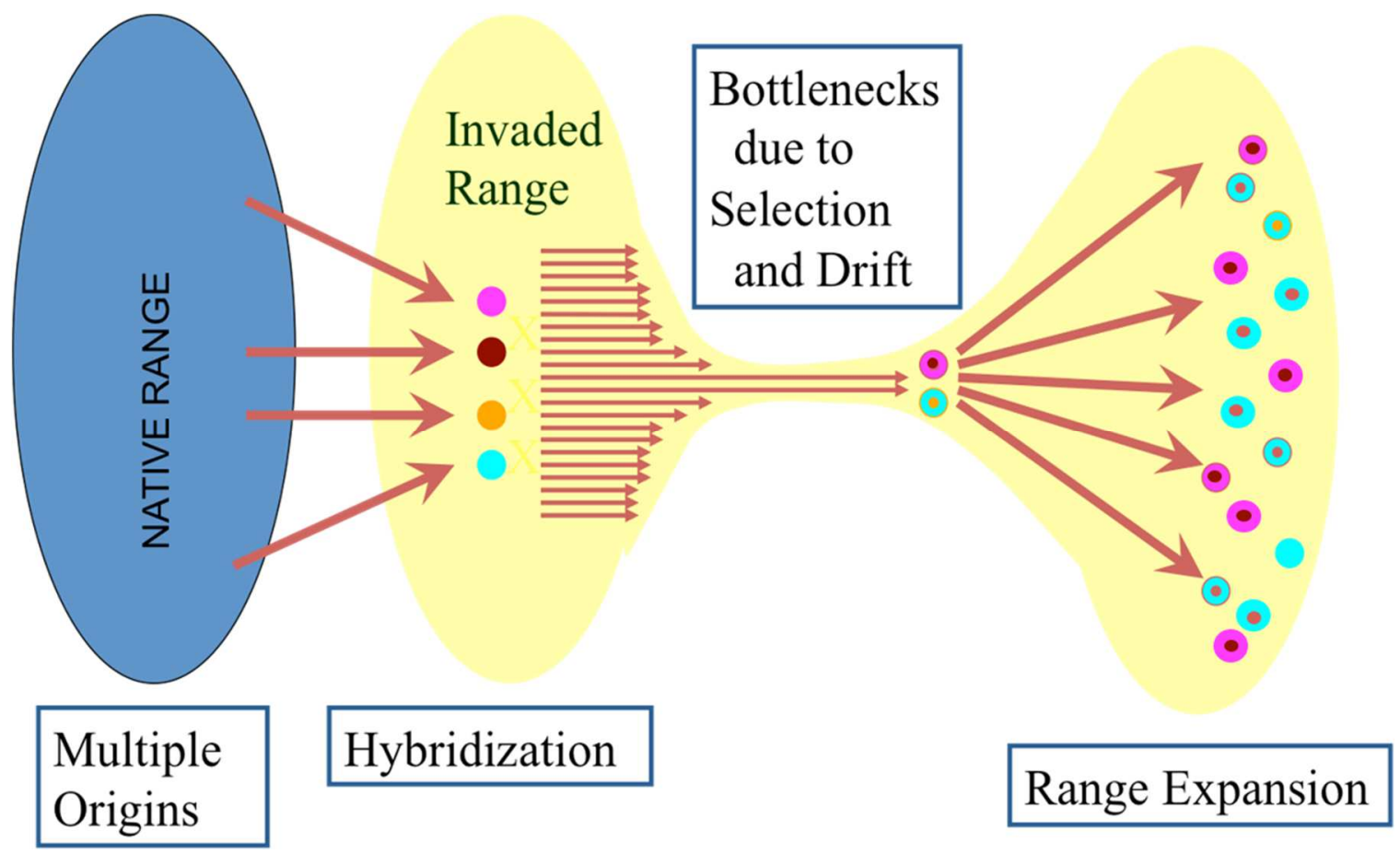




\title{
Chapter 2
}

Rapid Purging of Genetic Load in a Metapopulation and Consequences for Range

\section{Expansion in an Invasive Plant}

\begin{abstract}
Invasive species often display high fitness despite bottlenecks and inbreeding during establishment. We address this paradox through simulations and experiments that assess the potential for purging of genetic load during range expansion. Success of invaders often depends on the production of vigorous inbred offspring allowing for rapid population growth after colonization. Substantial genetic load of outbreeding species reduces the fitness of offspring as inbreeding ensues during the establishment of populations. In our simulations, sustained selfing or outcrossing within isolated populations did little to remove deleterious mutations. Conversely, inbreeding combined with periodic gene flow resulted in efficient purging and accelerated rates of range expansion. Purging efficiency was dependent on initial genetic diversity levels, in line with predictions that multiple introductions facilitate invasion and the evolution of more aggressive invaders. Simulation predictions were tested using the invasive species Brachypodium sylvaticum. Homozygous populations on B. sylvaticum's range periphery displayed lower inbreeding depression compared to heterozygous populations near introduction sites. Empirical tests with B. sylvaticum demonstrate that purging of genetic load is a plausible scenario promoting range expansion during invasion.
\end{abstract}




\section{Introduction}

Inbreeding within populations increases homozygosity and the expression of genetic load - the accumulated complement of deleterious recessive alleles that is often prevalent in outcrossing species. Inbreeding exposes deleterious alleles to the effects of selection and may reduce population growth and persistence (Crow and Kimura 1970; Keller and Waller 2002). Invasive species provide an ideal model for studying the effects of inbreeding on establishment and range expansion, as spreading populations of alien species often possess a low genetic diversity resulting from bottlenecks and small population size (Frankham 2004). Inbreeding depression, defined as the loss of fitness in an inbred individual compared to an outcrossed individual, results from genetic load (the sum of accumulated deleterious recessive mutations). Expression of genetic load due to inbreeding can slow population growth (Endels et al. 2007; Charlesworth and Willis 2009) and decrease the rate of range expansion (Excoffier et al. 2009; Fig. 2.1). In contrast to these expectations, many invasive species display vigorous population growth and range expansion throughout invasion (Frankham 2004). Based on the success of invasive species in novel environments, we would expect that the loss of genetic load may be occurring during range expansion (Whitlock and Bourguet 2000).

Range expansion during invasion of novel geographic regions may facilitate purging, a process where genetic load is reduced by selection against the recessive deleterious alleles expressed in the homozygous state (Pujol et al. 2009; Barringer et al. 2012). This hypothesis has been previously tested through creation of serial inbred lines or artificial bottlenecks to force genetic purging under controlled laboratory settings (Byers and Waller 1999; Crnokrak and Barrett 2002; Leberg and Firmin 2008), however 
examples of wild populations with the substantial variation in levels of inbreeding depression signaling that purging has occurred are rare (e.g., Haikola et al. 2001; Goodwillie and Knight 2006; Pujol et al. 2009; Barringer et al. 2012). Utilization of a recently-introduced invasive species with a well-described invasion history to investigate the process of genetic purging is ideal, as individuals can be collected from the field and allowed to cross in a controlled environment. Measurement of the fitness of outcrossed and selfed progeny then provides a direct test of levels of genetic load characterizing populations and whether genetic purging is occurring in wild populations.

Range expansion during invasion occurs as propagules migrate from source populations to form small founder populations. This process of range expansion continues until the invasion is a network of interconnected small and large populations, forming the invasive metapopulation. In small populations genetic drift can outweigh selection resulting in the fixation of a high proportion of deleterious alleles (Hedrick and Kalinowski 2000). In large, outbreeding populations genomic heterozygosity remains high, so genetic load is not expressed. Colonization processes during range expansion may exacerbate the negative effects of genetic load, as evidenced by high levels of inbreeding depression due to increased homozygosity in small populations. Gene flow to small populations increases heterozygosity as it infuses isolated populations with genetic variation, which increases selection efficiency, elevates fitness, and promotes further range expansion of the invasive species (Fig. 2.1; Richards 2000; Keller and Waller 2002). Intermittent gene flow to populations can facilitate genetic purging, as the periods in between gene flow events would allow selection to favor high-fitness alleles at loci that have become fixed for deleterious mutations. We hypothesize that intermittent gene 
flow (low levels of gene flow) is more likely to result in genetically purged populations because high rates of gene flow results in many heterozygotes and can swamp the effects of selection on populations. Conversely, intermittent gene flow to isolated populations allows time for selection to occur, removing unfit homozygotes and leaving homozygotes with high fitness.

We propose that purging of genetic load may be promoted by periodic gene flow to isolated populations, leading to increased fitness and accelerated range expansion. Periodic gene flow reflects processes occurring during range expansion as populations are likely to be isolated for some generations after colonization (Ramakrishnan et al. 2010). Population bottlenecks during range expansion can lead to inbreeding depression, allowing strong selection to eliminate deleterious alleles. Multiple generations of inbreeding in small populations without gene flow should expose a larger proportion of deleterious alleles to selection resulting in more effective purging. We perform an empirical test of the prediction that populations with lower genetic diversity may have higher fitness due to genetic purging through comparison of the fitness of selfed and outcrossed progeny from central and peripheral populations of the newly-invasive bunchgrass, Brachypodium sylvaticum (Hudson) Beauv. (slender false brome; Poaceae).

We developed three simulations to describe mechanisms by which genetic purging may occur in wild populations of B. sylvaticum. Our goal was to start with simplistic conditions, building on the outcomes to add more realistic scenarios in the second and third simulations. Our first simulation explored the hypothesis that populations of primarily selfing individuals outcrossing intermittently were more likely to result in genetic purging than a population of completely selfing individuals. We 
examined the effects of three variables on fitness: effective population size $\left(N_{e}\right)$, the number of unlinked loci contributing to inbreeding depression, and the frequency of outbreeding.

In our second simulation we expand on the previous hypothesis from a single population to a metapopulation, simulating gene flow across multiple inbreeding populations. In this simulation, we test the hypothesis that intermittent gene flow within a metapopulation facilitates genetic purging more effectively than a single large population or isolated populations consisting of selfing or outcrossing individuals. We also introduce mutation by allowing for one deleterious allele to appear at an anonymous location in each genome each generation (Denver et al. 2004; Haag-Liautard et al. 2007).

The third simulation integrates range expansion of the metapopulation to previously unoccupied habitats, testing the hypothesis that purging can occur simultaneously in more than one population of the invaded range, and that the occurrence of purging in invasive populations accelerates the rate of range expansion into previously unoccupied habitats.

\section{Materials and Methods}

\section{Simulations - Outcrossing Among Highly Selfing Lineages}

For the first set of simulations we explored the effects of the number of progeny (strength of selection, Fig. A.A2) and the number of loci contributing to genetic load on the effectiveness of genetic purging. To reflect patterns of colonization during range expansion we assumed that new populations would be established through selffertilization of a single colonizing individual. In this first simulation we mimicked a scenario where individuals primarily reproduced by selfing but would periodically 
outcross with other inbred lineages.

We used a collection of selfing lineages (represented by individual genotypes), annual hermaphroditic species that persisted in a single population with no outcrossing. We assumed the primarily selfing plants would infrequently outcross with other lineages in the same population. Generations were non-overlapping and discrete in this and all subsequent simulations. All lineages were initially heterozygous for $k=1$ to 1,000 unlinked loci segregating for two alleles, one deleterious $(a)$, the other of which produced a normal phenotype $(A)$. Deleterious alleles were assumed to be completely recessive to accommodate a dominance model for inbreeding depression (Charlesworth and Charlesworth 1987; Barrett and Charlesworth 1991). Genotypes across loci were randomly generated with $g_{a}=0.5$; i.e., approximately $25 \%$ of loci were homozygous for the deleterious allele in each lineage at the start of each simulation, where $g_{a}$ indicates the proportion of loci with the $a$ allele present. Absolute fitness $(W)$ declined proportionally with the number of loci homozygous for the deleterious allele ( $W=1-G_{a a}$; where $G_{a a}$ is the genomic frequency of loci homozygous for the $a$ allele), so effects across loci were additive and each locus had an equal effect on fitness.

Each generation, alleles at each locus of diploid individuals segregated to produce gametes that were randomly combined with another gamete from the same (selfing), or a different (outcrossing/gene flow) randomly-chosen lineage to produce a set of $n$ progeny ( $n=1$ to 1000$)$. We assumed that selection only occurred at the juvenile stage, so each lineage was replaced by one of its own offspring each generation. Selection was imposed by choosing the progeny genotype with the highest absolute fitness $(W)$ from among the $n$ progeny produced by each lineage in each generation (see Fig. A.A1). For these 
simulations we assumed the mutation rate was zero.

\section{Simulations - Gene Flow in a Metapopulation}

In the second set of simulations we examined the effects of mutation (introduction of deleterious alleles) and gene flow among $\tilde{N}$ inbred populations consisting of $N$ outbreeding individuals on levels of purging. Here, we assumed each population was established after dispersal of a single seed to an open site. Colonists were homozygous for deleterious alleles at $25 \%$ of their loci as described above. The individuals in each population were generated by selfing of each colonist in generation zero as described above. In subsequent generations the $N$ individuals were produced by outcrossing within each population. Selection was imposed by choosing progeny with the highest $W$ from among the $n$ progeny as described above. In this simulation we focused on the effects of gene flow among established populations so no extinction of lineages or populations was allowed (survival thresholds were set to zero - see Fig. A.A1).

A total of $k=100$ loci were monitored and were treated as anonymous so each locus could be representative of any location in the genome. One mutation per genome each generation was introduced at a random locus homozygous for the normal $(A)$ allele by generating a heterozygous genotype (the availability of anonymous locations homozygous for $A$ alleles never became limiting in the simulations described below). The rate of one deleterious mutation per genome per generation $(U)$ falls within the range

of estimates found for non-human animal species (Denver et al. 2004; Haag-Liautard et al. 2007), and is similar to estimates for most plants (0.14-2.0; Ossowski et al. 2010).

Simulations were conducted to examine the effectiveness of gene flow among inbred populations for purging genetic load $\left(g_{a}=0.50\right)$ with mutation and selection 
pressure. We examined the consequences of gene flow (every fifth generation) within a metapopulation $(\tilde{N}=10$ populations of $N=10$ lineages $)$ contrasted the effectiveness of purging of genetic load with a single large population $(N=100)$, the same set of ten populations with outcrossing and no gene flow, and the same set of ten populations with only selfing and no gene flow. We quantified purging of genetic load and changes in genotype frequencies for the average proportion of loci in lineages that were heterozygous or homozygous for each allele across populations. These population averages provide a measure of within-population purging and population fitness.

\section{Simulations - Metapopulation Range Expansion}

We modified the previous metapopulation simulation to examine rates of range expansion by creating a $20 \times 20$ array of sites that could be occupied by populations. In all simulations the invasion was initiated with five populations located at the center of the range, and a single selfing colonist as described above populated each site. Once populations exceeded the repopulation threshold then randomly-chosen individuals that exceeded the threshold were chosen to contribute to repopulation in populations that had less than ten individuals. Colonization occurred every second generation for sites that were adjacent to a population where the average fitness of lineages exceeded the colonization threshold. Colonization proceeded from propagules generated by randomlychosen individuals that exceeded the repopulation fitness threshold. Once a population was colonized it could grow to a population size of ten by production of progeny from within the population (if the fitness was greater than the repopulation threshold) or from an adjacent population (if the average fitness exceeded the colonization threshold).

Rates of range expansion were modulated by choosing fitness thresholds $W=0.60$ 
for individual survival, and individual average thresholds of $W=0.90$ for population growth and colonization. As lineages went extinct they could be replaced by high-fitness lineages from within the same population or from an adjacent population. With the fitness thresholds used we did not observe any population to suffer complete extinction. In all other respects the conditions used in this simulation matched those in the second simulation with the same levels of mutation and gene flow among populations, and complete outcrossing among individuals within each population.

We tracked average fitness of each population and number of sites occupied over 100 generations. To obtain different levels of differentiation among populations we generated sets of initial colonists that were identical for different proportions of their genomes (i.e. from zero loci identical to all $k$ of the loci identical among colonists of the $\tilde{N}$ populations). Genotypes at shared loci were randomly generated with $g_{a}=0.5$. We estimated levels of differentiation among the populations during the first generation of each simulation by calculating $F_{S T}$ for each locus and averaging across all $k$ loci (Hartl and Clark 2006).

\section{Empirical Tests - Population Inbreeding Depression}

Study species

Brachypodium sylvaticum is a diploid, perennial, C-3 bunchgrass native to Europe, Asia, and Africa, with invasive populations in North America and Australia. False brome was introduced in the Pacific Northwest of North America during the early 1900 's, but did not begin rapidly spreading in central Oregon's Willamette Valley until the 1980's (Rosenthal et al. 2008). This species continues to expand its range, and invasive B. sylvaticum can now be found in Washington, California, Virginia, New York, 
USA, as well as in British Columbia and Ontario, Canada (Rosenthal et al. 2008; Roy 2010). In Oregon this species is currently undergoing rapid range expansion. Recentlycolonized peripheral populations have lower levels of heterozygosity than populations close to the original introduction points near Corvallis and Eugene (Rosenthal et al. 2008a; Ramakrishnan et al. 2010).

\section{Data Collection}

We collected seeds to study patterns of genetic diversity of B. sylvaticum from 12 sites within central Oregon, USA to evaluate fitness-related traits under common garden conditions. Seeds were cold stratified in a $4^{\circ} \mathrm{C}$ refrigerator on Petri dishes for 28 days. If there was cotyledon emergence after stratification, seeds were planted into $162 \mathrm{~cm}^{3}$ pots. Once plants were grown, single tillers from at least eight plants per population were separated out and replanted into individual $162 \mathrm{~cm}^{3}$ pots into Pro-Mix soil (Premier Tech Horticulture, Quakertown, PA, USA). Plants were arranged in a randomized complete block design in a field environment on the campus of Portland State University in May 2008 and irrigated to supplement natural precipitation. Plant height, tiller and inflorescence number were measured once monthly for six months to assess fitness ( $\mathrm{n}=$ 33 to 50 per population). Eight plants per population were utilized for gene diversity estimates. Total plant size was calculated as the product of plant height and tiller number. Germination rate was calculated as the number of seeds germinated out of 20 after a twoweek period. Differences in germination rate were analyzed with a mixed model ANOVA in SAS 9.2 software.

We estimated levels of inbreeding depression for six of the populations that were used in the common garden study to represent peripheral and centrally located 
populations (three populations each). Seeds were gathered in the field from the chosen population sites and cold-stratified for 28 days in a $4^{\circ} \mathrm{C}$ refrigerator before being planted in the greenhouse. One tiller from each of ten individuals per population was separated and grown in a $162 \mathrm{~cm}^{3}$ pot. These plants were arranged in randomized blocks and allowed to freely self or cross-pollinate. Blocks were made up of maternal individuals from all six populations as well as individuals from 20 worldwide populations of $B$. sylvaticum. Mature seeds were collected and cold stratified. Six seeds per maternal plant were each planted in separate $162 \mathrm{~cm}^{3}$ pots in Pro-Mix soil (Premier Tech Horticulture). Plants were randomly placed within groups in a greenhouse in a randomized complete block design of 36 blocks ( 10 plants/block) to control for environmental variation across the greenhouse bench. Plant height, tiller, and inflorescence number were measured once monthly for three months for plant growth estimates with regular irrigation and fertilizer applications under controlled greenhouse conditions $\left(24 / 18^{\circ} \mathrm{C}\right.$, day/night, $10 / 12 \mathrm{~h}$ under ambient light). Additionally, twenty seeds per maternal plant were used to assess early life stage inbreeding depression through germination by being placed in Petri dishes on moist germination paper and checked daily for signs of emergence, at which point they were considered to be germinated.

We measured fitness of individuals as the multiplicative product of seed germination, size at one month, size at six months, survival, and biomass, divided by the maximum value to scale fitness from $0-1$. Seedlings from each maternal parent were determined to be products of self- or outcross-fertilization (selfed or outcrossed) from 12 microsatellite markers. A seedling was determined to be the product of self-fertilization if alleles at all eleven loci matched maternal alleles (see Fig. A.A3). Inbreeding depression 
was calculated as the decrease in fitness between selfed and outbred plants from the same maternal plant. We analyzed what factors influenced fitness differences within populations with a mixed model ANOVA in SAS 9.2. We included "population", "block", "selfed/outcrossed" (S/O), "population" nested within "maternal plant" and the interaction of "population" and S/O in our model, treating "maternal plant" and "block" as random factors.

\section{Results}

\section{Simulations - Outcrossing Among Selfing Lineages}

Loss of deleterious recessive alleles in selfing lineages depended on the population size, the strength of selection, and the number of loci segregating for deleterious alleles (Fig. 2.2). Purging was least effective with continual self-fertilization (Fig. 2.2a,b; $N=1$ lineage) as lineages quickly became fixed for deleterious alleles at a larger proportion of their loci compared to lineages that periodically outcrossed. Fixation of deleterious alleles decreased rapidly (i.e., fitness increased) from continually selfing lineages $(N=1)$ up to five lineages, but changed much less for population sizes greater than $N=5$. The strength of selection (effects of progeny number, $n$ ) increased rapidly when $n<25$, but purging efficiency was marginal for increases in the number of progeny from 25 to 200, especially with larger numbers of lineages (Fig. 2.2a,b). The proportion 
of loci homozygous for deleterious alleles was greater (fitness declined) when the number of loci was moderate to large and the number of lineages was smaller (Fig. 2.2c,d). The effectiveness of purging was improved and the average fitness of populations was substantially increased with repeated selfing followed by outbreeding every five generations (Fig. 2.2a,b). In all cases the fixation of normal alleles across lineages lagged behind the average frequency of fixation within lineages. Outbreeding contributed to this process as individual lineages become fixed for high-fitness alleles, then these highfitness alleles spread among lineages through continued crossing among lineages.

\section{Simulations - Gene Flow in a Metapopulation}

The effects of gene flow among inbreeding populations on loss of deleterious alleles in a metapopulation were similar to patterns of purging in selfing lineages. The degree of purging depended largely on metapopulation structure and the frequency of gene flow (Fig. 2.3). A single large population of 100 lineages rapidly became homozygous for deleterious recessive alleles for around $25 \%$ of loci (Fig. 2.3a). This degree of purging was similar for a metapopulation consisting of the same total number of lineages divided into ten isolated populations of ten lineages that only outcrossed within each population (Fig. 2.3b). Similarly, purging was less effective for a metapopulation of completely selfing lineages that lacked gene flow where around $40 \%$ of loci became fixed for deleterious alleles (Fig. 2.3c). The most effective purging was observed when lineages outcrossed within populations and gene flow occurred every fifth generation among populations (Fig. 2.3d). Under this scenario no loci became fixed for deleterious alleles and around $90 \%$ of loci became homozygous for normal alleles. Close to $10 \%$ of loci remained heterozygous in outcrossing populations due to new mutations 
(Fig. 2.3a, b, and d), but the proportion of heterozygous loci maintained in selfing populations was much less (Fig. 2.3c). Introducing mutations at a rate of one per genome per generation contributed to the maintenance of genetic variation within populations and prevented metapopulation-wide fixation of alleles for all four scenarios depicted in Fig. 2.3

The maintenance of genetic variation and the effectiveness of purging depended on metapopulation size (see Fig. A.A4). Increasing the number of populations undergoing periodic gene flow allowed for a higher proportion of loci to become fixed for the normal allele from around $65 \%$ (one population) to $90 \%$ (for $>$ ten populations). Higher levels of genetic variation were maintained in larger metapopulations as none of the loci became fixed for either allele with more than ten populations. The introduction of new mutations maintained heterozygosity around $8 \%$ to $11 \%$ across a range of metapopulation sizes (see Fig. A.A4).

\section{Simulations - Metapopulation Range Expansion}

With spatially-restricted gene flow and colonization of open sites range expansion proceeded slowly for the first 25 generations, but became much more rapid as populations became purged of their genetic load (Fig. 2.4). When simulations were initiated with no shared loci among the colonists the process of purging began as open sites received seed dispersal from more than one neighboring site (note the single orange population; Fig. 2.4a). Episodes of purging often began a multiple locations as the range slowly expanded (Fig. 2.4b), and subsequent colonization came primarily from the populations with higher fitness (Fig. 2.4c). As the expanding fronts of higher fitness genotypes from independent purging events coalesced, additional rounds of purging were 
initiated and contributed to more rapid range expansion (note the darker red populations between the expanding ranges from the original purging events; Fig. 2.4c,d). The degree of purging and subsequent rate of range expansion was heavily dependent on the relatedness (genetic diversity) among the initial colonists (Fig. 2.5); invasions initiated by colonists representing higher levels of genetic diversity caused rapid purging and exponential range expansion, while range expansion was much slower when initial colonists were more closely-related.

\section{Empirical Tests - Inbreeding Depression}

Observed populations heterozygosity $(H s)$ was calculated for each population as the average heterozygosity of individuals within the population. $H s$ ranged from almost completely homozygous ( $H s$ values ranged from 0.04 to 0.09 for homozygous populations E4, M1, and S2) to highly heterozygous ( $H s$ values ranged from 0.13 to 0.39 for heterozygous populations $\mathrm{C} 6, \mathrm{C} 7, \mathrm{C} 1, \mathrm{C} 4, \mathrm{E} 1, \mathrm{C} 2, \mathrm{C} 10$, and E9; Fig. 2.6). Populations with the highest genetic diversity tended to be located towards the central regions of the invasion, while more homozygous populations were located at the edge of the invasive range. In general, offspring from inbred populations performed poorly under field cultivation, but plants from one highly homozygous population (E4 in Fig. 2.6) had growth rates similar to offspring from genetically diverse populations, suggesting reduced genetic load. The results from our ANOVA showed significant effects of "block" $\left(F_{11.825}=11.2, p<0.001\right)$, initial plant size $\left(F_{1,825}=19.7, p<0.001\right)$, and homozygous and diverse populations $\left(F_{10,825}=4.13, p<0.001\right)$ on vegetative biomass of individuals (Fig. 2.6).

Selfed and outcrossed offspring from homozygous populations displayed high 
levels of fitness and little or no evidence of inbreeding depression under controlled greenhouse conditions; while the fitness of seedlings from the central and more genetically diverse populations was much lower and displayed greater levels of inbreeding depression (Fig. 2.7). The probability of undetected outcrossed offspring to be quite low (Maximum $P(u)_{j}=4.37 \times 10^{-6}$ ). We found significant effects of $\mathrm{S} / \mathrm{O}$ fertilization $\left(F_{1,199}=6.39, p=0.01\right)$ across populations, the identity of the source population $\left(F_{5,46}=51.3, p<0.001\right)$ and due to variation among blocks $\left(F_{36,199}=1.57, p=\right.$ 0.03) on multiplicative fitness. A significant effect of S/O by population interaction $\left(F_{5,199}=2.49, p=0.03\right)$ was found in the same model indicating that the intensity of inbreeding depression varied among populations. There was no significant effect of maternal individual on multiplicative fitness $\left(F_{38,199}=1.30, p=0.13\right)$.

\section{Discussion}

Our simulations indicate that cyclic inbreeding and outbreeding among populations can purge genetic load more effectively than sustained selfing or inbreeding within small populations. These cycles mimic inbreeding and gene flow that occur with colonization and dispersal during range expansion. Genetic purging within populations of an invasive plant can result in high-fitness populations at the range edge, instigating new phases of rapid and aggressive invasion as purged individuals establish in new sites and contribute to gene flow among established populations.

The result from our simulations and empirical study are applicable to developing more effective strategies determining patterns of invasion and determining population characteristics that result in invasive success. The restricted population sizes that are common to the step-wise dispersal accompanying species invasion (Pujol et al. 2009) 
are similar to cases of rare species where fragmented and isolated populations occur throughout the species' range. Artificially imposing gene flow among inbred populations would infuse new variation for more effective purging of genetic load, and may provide an effective alternative to the inbreeding strategies that are currently being employed (Hedrick and Fredrickson 2010). Our results suggest that alternating cycles of inbreeding and gene flow among inbred populations may be an effective strategy for the recovery of species suffering from inbreeding depression. On the other hand, the effectiveness of purging depends on the relatedness among populations that are interbred; admixture among disjunct populations representing divergent lineages will provide more effective purging (e.g., Johnson et al. 2010).

Based on results from our simulations the process of purging genetic load appears to be dependent on inbreeding to facilitate the exposure of deleterious mutations to selection coupled with low levels of gene flow to introduce adequate genetic variation at loci that had become fixed for deleterious alleles. These conditions may frequently occur during the establishment and spread of invasive species. During range expansion sites at the range periphery are typically colonized by one or a few individuals (Ibrahim et al. 1995; Sakai et al. 2001), so new populations may be subject to inbreeding for several generations before additional genetic variation is introduced via gene flow (e.g., Ramakrishnan et al. 2010). As the range expands, groups of populations near the expanding limits of the range may be subject to cycles of inbreeding followed by infrequent gene flow among populations, which creates conditions that promotes purging and the emergence of high-fitness populations that accelerate the rate of range expansion.

The efficiency of purging in our simulations depended largely on metapopulation 
structure and initial levels of genetic diversity. As individual lines or populations inbreed they become nearly homozygous within a few generations, and depending on the strength of selection, a somewhat higher proportion of loci become fixed for high-fitness alleles. More loci are made heterozygous with each cycle of outbreeding, which provides opportunities for sequential rounds of selection to incrementally decrease the number of loci segregating for deleterious alleles. At the end of this process all of the lines achieve higher fitness, but may still be fixed for a subset of deleterious alleles. Opportunities for additional purging would arise with gene flow from neighboring populations, which would introduce additional variation so the process could begin anew. For example, note the additional purging that occurred when populations from different expanding ranges met (darker red populations appearing in Figs. 2.4c and d). The rate and efficiency of purging in a metapopulation with cyclic inbreeding were similar to predictions from analytical models finding that the mean fitness of a population is dependent on population subdivision (Whitlock 2002), but our simulations and experiments extend these results to accommodate conditions that are likely to occur during the establishment and invasive by introduced species.

While our simulations reveal a mechanism for purging of genetic load they include a number of simplifications that might affect the generality of the results. For example, these models do not allow for the influence of environmental variation on fitness or demographic stochasticity. Both of these factors would be expected to mute the effects of selection and enhance genetic drift in populations (Hartl and Clark 2006). These nongenetic sources of variation would probably slow the rates of purging, but the general outcomes of our simulations should hold. Similarly, the range of selection values that we 
examined was limited ( $s \approx 0.04$ to 0.12 for progeny arrays of $n=2$ to 100 ; Fig. A.A2). Our metapopulation simulations were conducted with $n=100$ and reducing the number of progeny per generation extends the time required for purging, but the results remain qualitatively the same as those reported here. It is also important to note that these models focus on the process of purging genetic load and do not address processes of adaptive evolution in novel environments, which is also thought to be more effective when the initial level of genetic variation in the colonizing population is high (Charlesworth and Willis 2009).

Other simplifications include the assumptions that deleterious alleles were completely recessive and had equal effects on fitness. Estimates from several species suggest a leptokurtic distribution of effects of mutations with a small number having larger effects on fitness (Lynch et al. 1999; Eyre-Walker and Keightley 2007). The dominance of mutations segregating in populations also varies and tends to be much weaker for alleles with larger negative effects on fitness (Halligan and Keightley 2009). However, both theoretical and empirical results of the dynamics of purging genetic load indicate that those mutations with larger effects and higher levels of expression in the heterozygous state are more easily eliminated from populations (Charlesworth and Willis 2009). By examining the dynamics of completely recessive alleles with very small effects on fitness these simulations provide a conservative view of purging dynamics; we would expect more rapid elimination of mutations with larger effects or those with some expression in the heterozygous state.

The efficacy of purging genetic load in these simulations and our experiments with $B$. sylvaticum are striking when contrasted with results from unsuccessful crossing 
experiments designed to eliminate deleterious mutations through lines of inbreeding or artificial creation of bottlenecks (Byers and Waller 1999; Crnokrak and Barrett 2002; Carr and Dudash 2003; Swindell and Bouzat 2006). One of the possible reasons for the difficulty of reducing genetic load in populations is due to the generation of gametic disequilibrium by inbreeding (Lande et al. 1994; Winn et al. 2011). Disequilibrium across the genome leads to selective interference because the effects of advantageous alleles at some loci may be counterbalanced by their association with deleterious alleles at other locations (Cutter and Payseur 2013; Neher 2013). Gene flow alleviates selective interference by providing opportunities for recombination to disassociate beneficial and deleterious mutations and elevates levels of genetic diversity within populations. The strong effect of gene flow among inbred populations for purging in our simulations is dependent on low relatedness among populations, which allows for recombination among divergent genomes. Our results help elucidate how the process of genetic purging can occur in nature resulting in potential for introduced species to evolve into aggressive invaders.

\section{Effects of Range Expansion on Genetic Load}

Populations across the expanding range of B. sylvaticum vary in observed heterozygosity, with more genetically depauperate populations located towards the leading edge of the expanding range (Rosenthal et al. 2008; Ramakrishnan et al. 2010). The performance of offspring from crosses within populations depends on both the history of inbreeding and the degree to which genetic load has been purged. In general, plants from genetically diverse populations close to introduction points outperformed plants from the range periphery under field conditions, but some homozygous 
populations with low genetic load represent outstanding departures from this pattern. The higher fitness of both selfed and outcrossed progeny from peripheral populations of $B$. sylvaticum suggests that reductions in load have occurred during range expansion. According to the results from our simulations, these high-fitness populations may represent the beginning of the next phase of the false brome invasion - one that will be much more rapid and aggressive as purged genotypes colonize new sites and contribute to gene flow among established populations.

There are some particular aspects of the histories of species that may explain why purging is more likely during invasion than for stable metapopulations of native species. False brome and many other invasive species have succeeded partly as a consequence of multiple introductions of genetically divergent individuals (Roman and Darling 2007; Dlugosch and Parker 2008; Facon et al. 2008; Rosenthal et al. 2008). Our simulations demonstrate that invasions initiated with higher levels of genetic divergence have more potential for elevating absolute fitness through the elimination of deleterious mutations contributing to genetic load. Multiple introductions of invasive species would create a metapopulation that more closely approximates the high genetic diversity scenarios in our simulations. Stable metapopulations of native species, on the other hand, are more likely to consist of populations from a single origin, so relatedness is higher and they are more likely to share a large proportion of fixed deleterious genetic variation. Higher levels of genetic diversity within metapopulations derived from multiple introductions create conditions that favor purging of genetic load through cycles of inbreeding and gene flow, and may be responsible for fitness increases in peripheral populations of B. sylvaticum. Multiple introductions could also carry novel genetic variation that facilitates adaptation, 
which could contribute to the success of invasive species (Novak and Mack 1995; Meimberg et al. 2010). In the case of B. sylvaticum the higher fitness we observed in peripheral populations occurred in a controlled greenhouse environment, so this increased fitness is more likely to be due to purging of genetic load.

Our simulation and empirical results have implications for the long-range prospects of the success and further evolution of invasives and other species undergoing to range expansion. Populations that have lost much of their genetic load through purging during range expansion are susceptible to the spread of mutations that increase the frequency of selfing (Jarne and Charlesworth 1993). As purging increases the fitness of inbred offspring, it effectively reduces the "cost" of selfing (Fisher 1941; Nagylaki 1976), which is expected to lead to the rapid evolution of primarily selfing lineages (Lande and Schemske 1985). This scenario presents a potential mechanism for the generation of new selfing lineages; extensive range expansions precipitated by human-mediated dispersal of species and climatic change create conditions that facilitate purging of genetic load and the evolution of selfing. Under such a scenario, the emergence of a new complement of selfing species may be imminent as human-mediated disturbance, long-distance dispersal, and rapid climate change lead to range expansions and purging of mutational load, conditions that favor the evolution of self-fertilization in hermaphroditic species.

In summary, our results from simulations and estimates of inbreeding depression in populations of the newly-invasive Brachypodium sylvaticum are consistent with the hypothesis that range expansion can facilitate rapid purging of genetic load in outcrossing species. The simulations further demonstrate that loss of genetic load is promoted by high levels of genetic diversity, which may be provided by multiple introductions from 
divergent populations in the native range. High levels of genetic variation during establishment and range expansion may promote adaptation to novel conditions in the introduced range (Chapter 4; Lavergne and Molofsky 2007), and may increase the overall vigor of invasive species through the purging of genetic load. In the case of $B$. sylvaticum in particular, purging of genetic load during range expansion may precipitate a more rapid and aggressive phase of invasion. Comparative analyses of levels of inbreeding depression in other invasive species will provide a more complete picture of the role of purging in the evolution of aggressively invasive species.

Acknowledgements We thank D. Butler, B. Hergic, J. Lawson, and M. Steele for technical assistance, and T. Cheeke, C. Lee, T. Musial, and R. Workman for comments on the manuscript. This work was funded by a Faculty Enhancement Grant from the Office of Academic Affairs at Portland State University to M.B.C., and by USDA grant \#2005-35320-15317 to M.B.C. and A.P.R.

At the time of dissertation submission, this article was in press under the citation: Marchini, G.L., N.C. Sherlock, A.P. Ramakrishnan, D.M. Rosenthal, and M.B. Cruzan. In press. Rapid Purging of Genetic Load in a Metapopulation and Consequences for Range Expansion in an Invasive Plant. Biological Invasions. DOI: 10.1007/s10530-015$1001-5$ 


\section{Tables and Figures}

Figure 2.1 A qualitative model for the dynamics of range expansion under selection and purging of genetic load in an outbreeding invasive species. Phase $1-$ a primary lag phase occurs during initial colonization and establishment as the invader responds to selection in a novel environment. Phase 2 - initial range expansion occurs as open sites are colonized. Phase $3-$ a secondary lag phase occurs due to inbreeding and population bottlenecks during the establishment of satellite populations. Purging of genetic load occurs through inbreeding and gene flow among satellite populations. Phase $4-$ exponential range expansion ensues as genetic load is purged. Newly-established populations on the range edge have high rates of growth because of the lack of inbreeding depression.

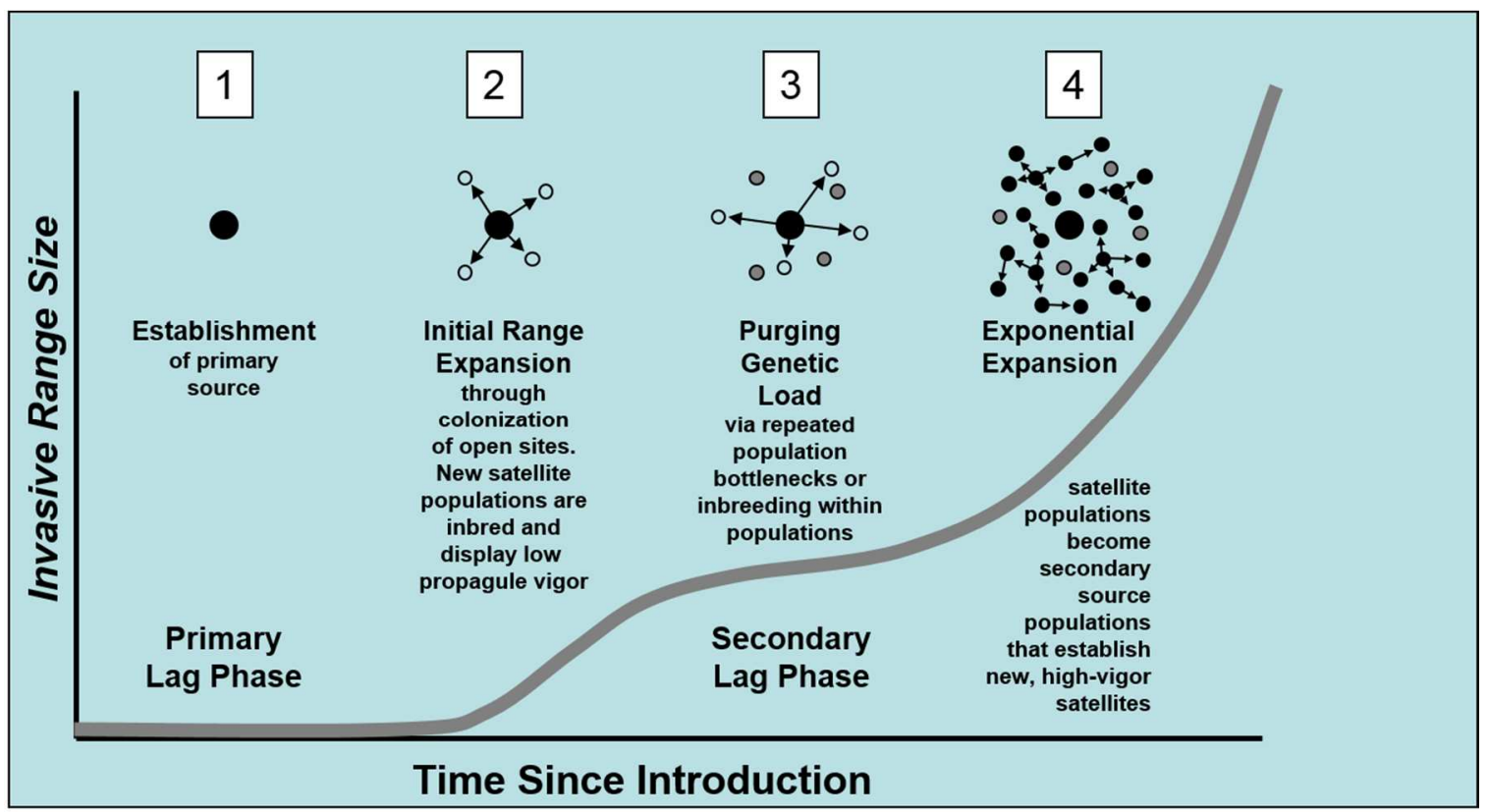


Figure 2.2 Effects of population size and number of loci on purging levels within lineages (Mean $G_{A A}$ ) and the proportion of loci fixed for normal alleles (Fixed AA) across lineages. The effects the strength of selection ( $n$ progeny $=1$ to 200$)$ are shown in the two top panels ( $\mathrm{a}$ and $\mathrm{b}$ ); where each line represents the number of lineages from $N=1$ lineage that selfed every generation or outcrossed every fifth generation $(N=2$ to 10 lineages). The effects of number of loci are shown in the two bottom panels (c and d); where each line represents $k=1$ to 1000 loci with their response to the number of lineages on the horizontal axis ( $n=100$ progeny). Generations were discrete and selection was based on replacing each lineage with the fittest progeny. Mutation rate is zero in all cases. Error bars were too small for accurate representation.

\section{Number of Lineages}

(a)

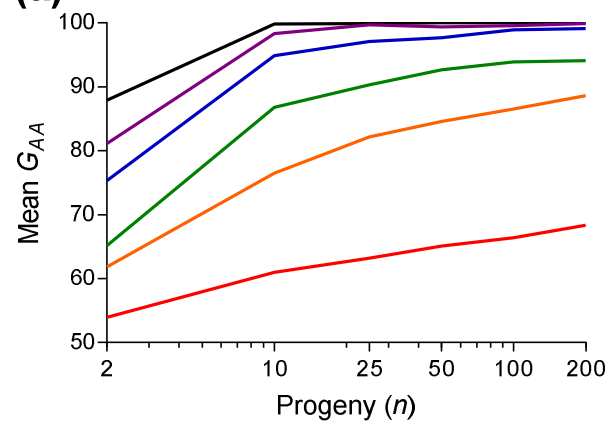

Number of Loci

\section{(c)}

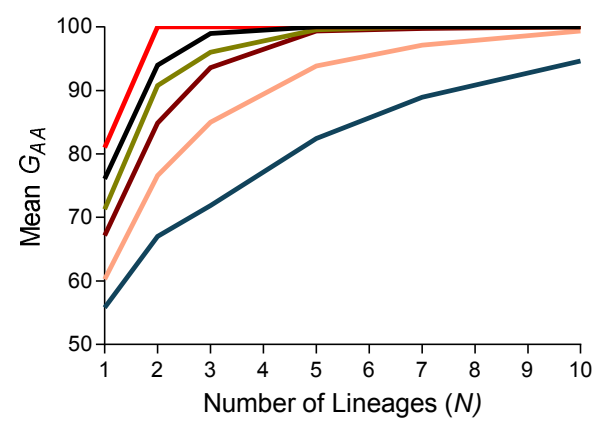

(b)

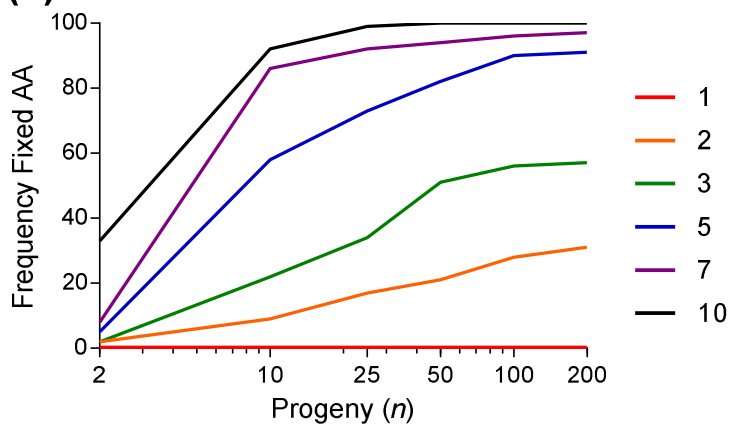

(d)

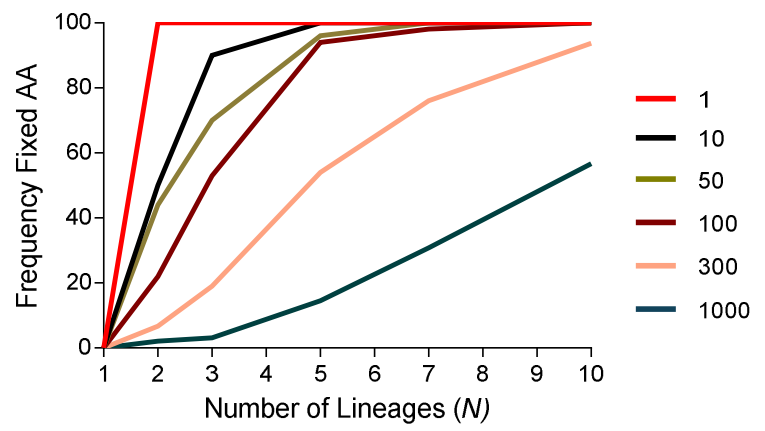


Figure 2.3 The effects of metapopulation structure, breeding system, and gene flow on changes in the frequencies of deleterious mutations. Outcrossing in a single large population of $N=100$ (a) is contrasted with the same total number of individuals divided up into $\tilde{N}=10$ isolated populations of $N=10$ lineages that only self (b; no gene flow) or only outbreed within populations (c; no gene flow), and ten populations of ten individuals that only outbreed within populations and experience gene flow with a randomly-selected population every five generations (d). Selection is based on $n=100$ progeny each generation for each line. Deleterious mutations occur at a rate of one per genome per generation with $k=100$ loci. Error bars were too small for accurate representation.

(a) One population of 100

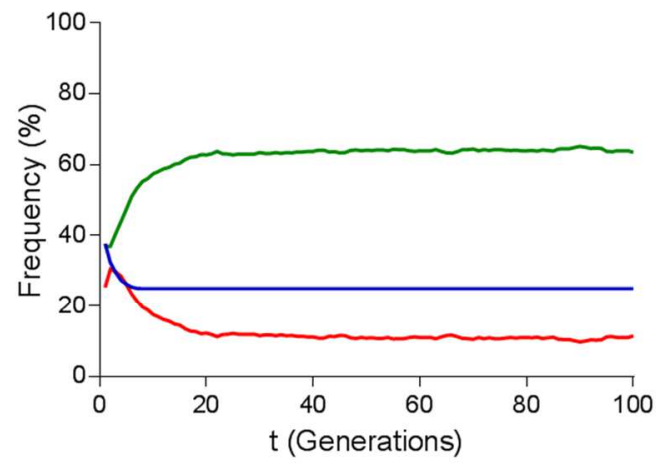

(c) No gene flow- selfing

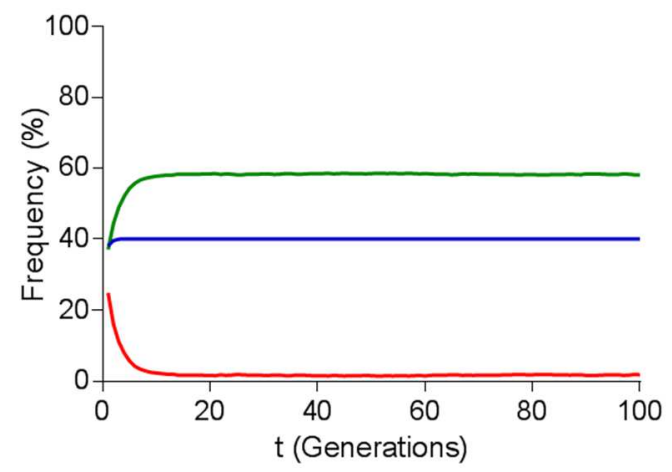

(b) No gene flow-outcrossing

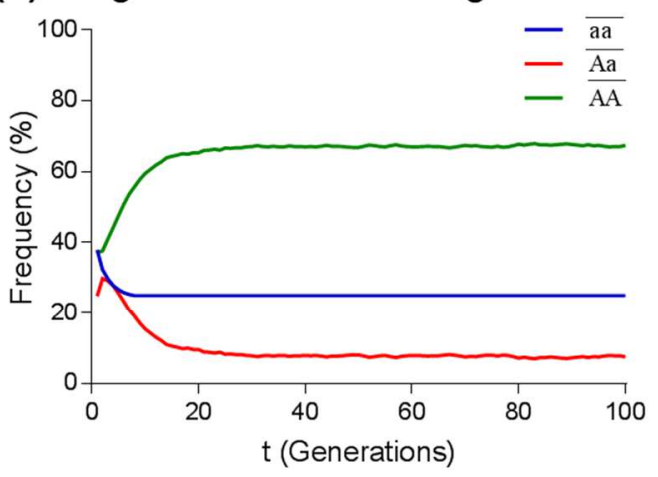

(d) Metapopulation with gene flow

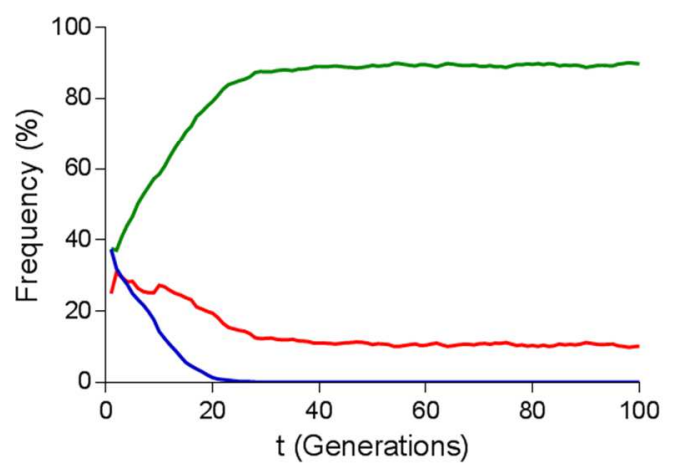


Figure 2.4 Stages of range expansion after establishment of an outbreeding invasive species; a) one of a few satellite populations displays higher fitness after colonization by more than one of the original five populations (dark blue outline); b) invasion continues as new sites are colonized primarily from the first high-fitness population. A second purged population appears on the opposite side of the range; $c$ ) rapid range expansion as satellite populations from the two original purging events undergo admixture. Another round of purging produces higher fitness populations; d) high-fitness genotypes spread and range expansion is rapid, purging is nearly complete. Simulation conditions: $F_{S T}=$ 0.47 among five colonizing populations in generation 1; population size $N=10$; selection based on $n=100$ progeny ( $s=0.12$ ); outcrossing within populations (no selfing); gene flow among populations every $10^{\text {th }}$ generation; fitness threshold for contributing to population growth or colonization $=0.90$; seed dispersal every second generation .

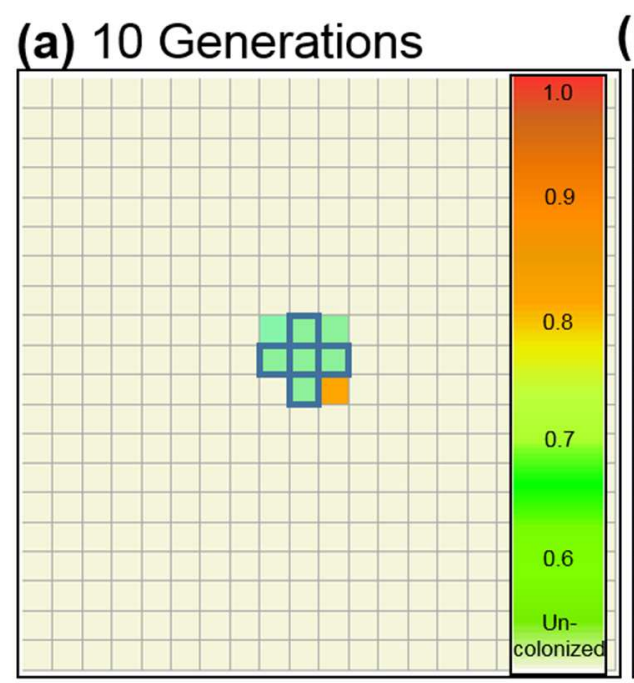

(b) 25 Generations

\section{(c) 50 Generations}
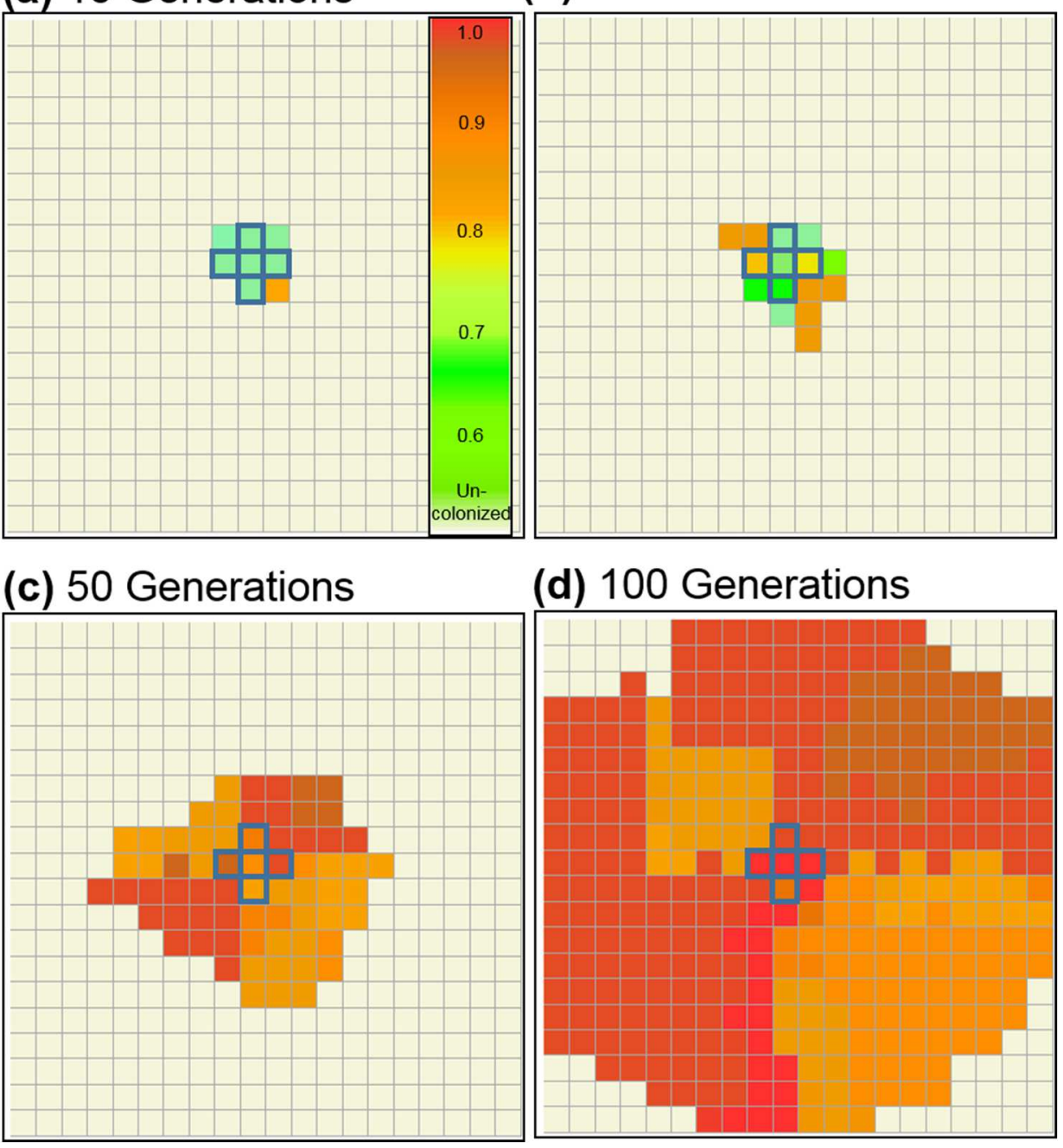

(d) 100 Generations

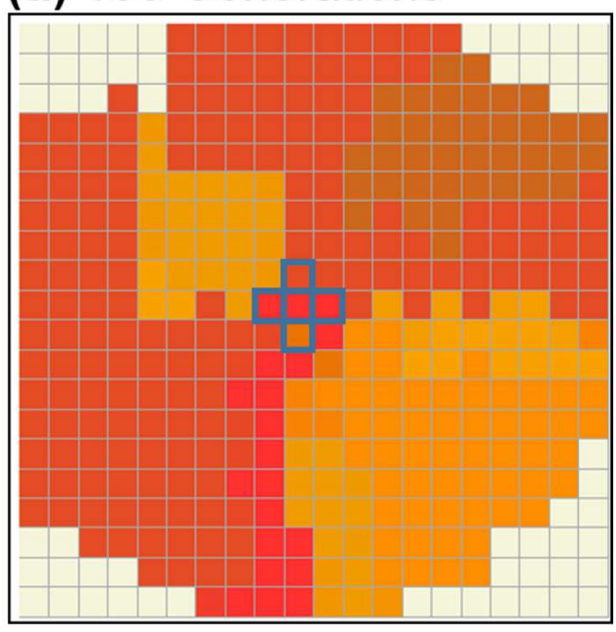


Figure 2.5 The effects of levels of divergence among established populations $\left(F_{S T}\right)$ on the potential for purging and rapid range expansion. All simulations were initiated under the same set of condition for the strength of selection ( $n$ progeny per generation).

Differences in $F_{S T}$ reflect the proportion of loci that were identical among the original colonists. With the exception of relatedness among colonists (initial genetic divergence), all simulation conditions were the same as for Figure 2.4.

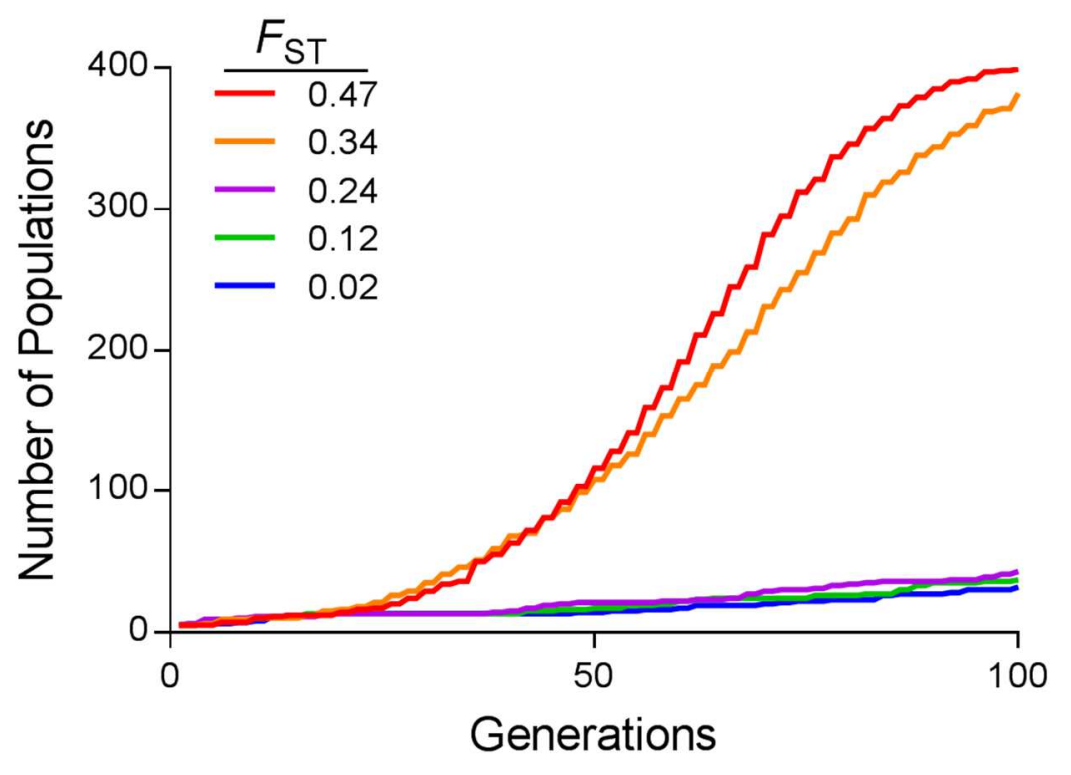


Figure 2.6 The relationship between population genetic diversity and plant biomass in a common garden. Homozygous populations from the range periphery are designated by blue squares; centrally located heterozygous populations are represented by green squares.

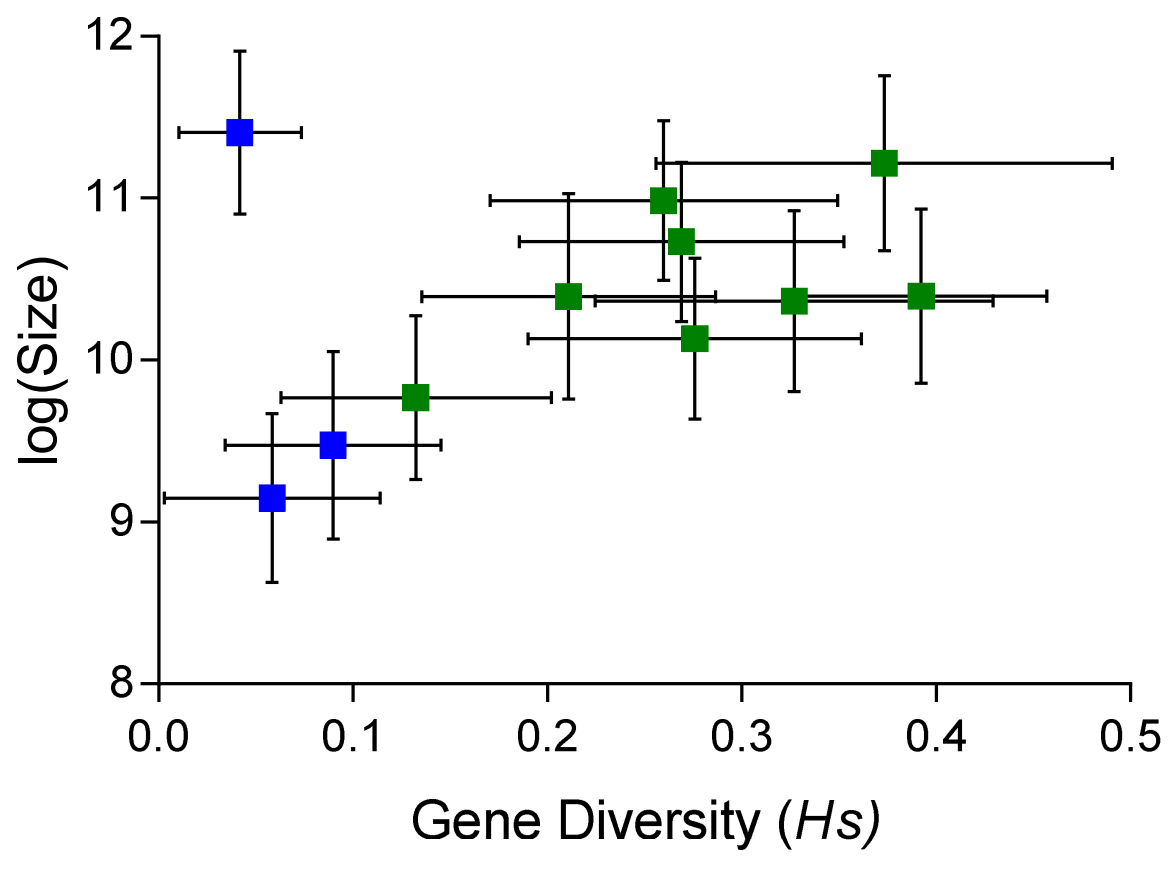


Figure 2.7 The fitness of selfed (spotted) and outcrossed (striped) progeny from populations that vary for genetic diversity (a), and the genetic diversity of source populations for outcrossed and selfed progeny (b) in the newly invasive grass, Brachypodium sylvaticum. (a) Letters denote groups of populations that are not significantly different in fitness (selfed and outcrossed progeny combined). Multiplicative fitness is estimated here as the product of seed germination, early growth, survival, and ranked biomass. Asterisks indicate a significant difference in fitness between selfed and outcrossed progeny within a population. (b) Genetic diversity of each population is represented by black circles.
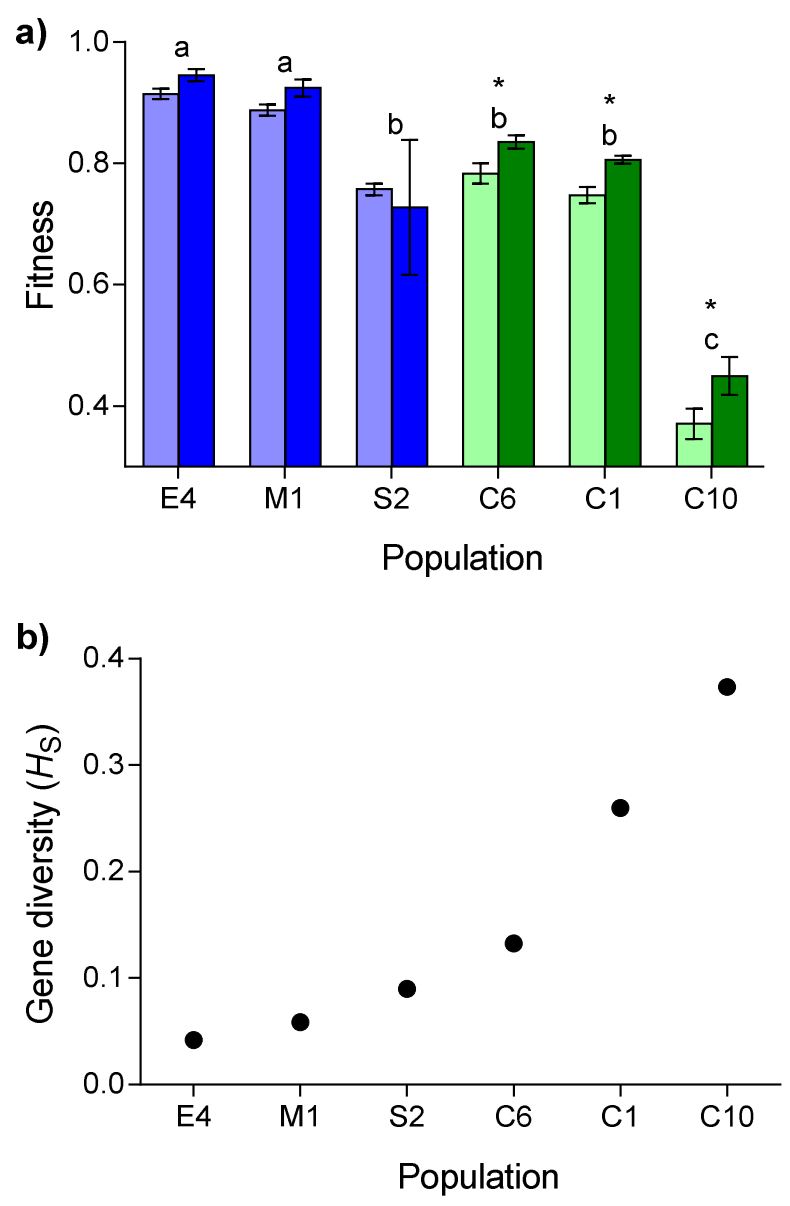


\title{
Chapter 3
}

Ecological mitigation of inbreeding depression in a newly invasive species

\begin{abstract}
The interaction of population genetic diversity and the environment has important effects on the spread and establishment of invasive species. Bottlenecks during range expansion limit genetic diversity and adaptive potential as a consequence of small effective population size and inbreeding. Homozygous individuals are more sensitive to environmental stress and may be less able to take advantage of available resources; however, adaptation to abiotic change such as increased nitrogen deposition may raise fitness of individuals from low genetic diversity populations, facilitating invasion. We utilized the rapidly- spreading invasive bunchgrass, Brachypodium sylvaticum, in a competition experiment to investigate how nitrogen addition influences performance of plants from low and high $H_{\mathrm{S}}$ populations. We found that nitrogen enrichment at levels consistent with aerial deposition due to human activities increases the size of individuals from low $H_{\mathrm{S}}$ populations but does not increase size of individuals from high $H_{\mathrm{S}}$ populations. Coefficients of relative competition intensity $(R C I)$ show no performance reduction of individuals from low $H_{\mathrm{S}}$ populations relative to individuals from high $H_{\mathrm{S}}$ populations. These results are consistent with ecological mitigation of inbreeding depression (EMID), which is the potential for increased resources to augment fitness in populations with limited gene diversity $\left(H_{\mathrm{S}}\right)$. Elevated nitrogen availability due to human activities is a factor that may be instrumental for increased invasion success of introduced species that are subject to inbreeding depression due to small effective population size.
\end{abstract}




\section{Introduction}

Range expansion into novel environments is a dynamic function of biotic and abiotic factors governing colonization success (Mack et al. 2000; Sakai et al. 2001). For plants, anthropogenic modifications can alter environmental factors such as temperature, nutrient availability, and ecological disturbance, increasing species turnover and successional changes in community composition that facilitate species invasions (Ibanez et al. 2009; Walther 2010; Moser et al. 2011). Populations can adapt to novel conditions during invasion but low genetic diversity may cause reductions in fitness and limit adaptive potential.

Bottlenecks occurring upon introduction cause newly invasive populations to have only a portion of the genetic diversity $\left(H_{\mathrm{S}}\right.$, population genetic diversity; Lee 2002$)$ of the source population. Estimates of $H_{\mathrm{S}}$ using neutral markers such as microsatellite loci provide information on historic population bottlenecks and levels of inbreeding in populations (Morgante and Olivieri 1993; Garza and Williamson 2001; Selkoe and Toonen 2006). Although many invasive species are established through multiple introductions, resulting in high $H_{\mathrm{S}}$ near sites of primary colonization, $H_{\mathrm{S}}$ in populations at the edge of the expanding range tends to be lower due to bottlenecks incurred during range expansion (GarciaRamos and Kirkpatrick 1997; Arnaud-Haond et al. 2006; Ramakrishnan et al. 2010). Lower genetic diversity and higher levels of inbreeding in newly established populations at the edge of the expanding range may lower population growth rate and stall the spread of newly-invasive species. 
Low $H_{\mathrm{S}}$ results from a historically small effective population size $\left(N_{\mathrm{e}}\right)$ that makes populations vulnerable to loss of genetic variation through genetic drift, demographic stochasticity, and inbreeding (Dlugosch and Parker 2008; Ramakrishnan et al. 2010). Commonly, a population isolated from gene flow for several generations will experience fitness decreases as drift results in random fixation of alleles (Young et al. 1996; Keller and Waller 2002; Excoffier 2004). Inbreeding depression, the reduction in fitness of inbred offspring in comparison to outbred offspring (Wright 1977; Charlesworth and Charlesworth 1987; Shields 1993), results from accumulation of deleterious alleles and loss of heterozygosity (Crnokrak and Barrett 2002). Drift and inbreeding reduce genetic variation and the potential for adaptive evolution, leaving a population vulnerable to environmental fluctuations (Keller and Waller 2002; Pearson et al. 2009).

Stressful environmental conditions, such as enhanced competition stemming from nutrient limitations, can exacerbate the negative effects of reduced $H_{S}$, resulting in environmentally dependent inbreeding depression (EDID; Fig. 3.1a) (Fox and Reed 2010; Cheptou and Donohue 2011; Bijlsma and Loeschcke 2012). However, despite the lack of genetic diversity resulting from demographic expansion, many species successfully spread and colonize new populations in the invasive range (Tsutsui et al. 2000; Dlugosch and Parker 2008). We propose that ecological mitigation of inbreeding depression (EMID; Fig 3.1b), an increase in the ability of low $H_{S}$ individuals to utilize available resources, underlies the ability of genetically depauperate invasive populations to colonize new areas.

Levels of inbreeding depression experienced by invading species during range expansion may be alleviated by favorable ecological conditions. In particular, increased 
resources afforded by human activities may provide ecological remediation of fitness declines normally observed for homozygous and inbred populations. We distinguish EMID from environmentally- dependent inbreeding depression (EDID; Cheptou and Donohue 2011) because it elevates the fitness of inbred populations to be equal to outbred populations when resources are more abundant (Fig. 3.1b). In contrast to EDID, under ecological mitigation low genetic diversity populations are more capable of taking advantage of increased resources then the outbred populations. Ecological mitigation of inbreeding depression could occur through strong selection that increases the frequency of genotypes that are locally adapted to abiotic conditions; either through selection against deleterious alleles that have accumulated in the homozygous state (genetic purging; Crow and Kimura 1970), or by selection on environmentally influenced phenotypes that are able to take advantage of nutrient availability (adaptive plasticity; Callaway et al. 2003; Pigliucci and Murren 2003), resulting in homozygous individuals possessing optimal trait values favored by the local environment. Under the EMID we predict that inbred invasive populations will be able to capitalize on increased resource availability (especially nitrogen) after environmental perturbations that damage or destroy native vegetation, and by increased aerial input of nitrogen due to human activities.

Nitrogen deposition is increasing due to rising levels of atmospheric pollution and may destabilize natural successional processes, providing opportunities for the establishment of invasive species (Callaway and Walker 1997; Ackerly 2003; Gilbert et al. 2009). The rate of input into the terrestrial nitrogen cycle has approximately doubled since the industrial revolution, dramatically increasing soil nitrogen levels that affect 
plant community composition (Vitousek et al. 1997). Many previous studies have found that plant communities are being significantly altered by atmospheric nitrogen depostion resulting from inorganic fertilizer use and nitrous oxide $(\mathrm{NO})$ emissions (Fenn et al. 2003). Increasing a fundamentally limiting soil nutrient such as nitrogen may create reductions in environmental stress that disrupts community dynamics by altering outcomes of competitive interactions (Gerry and Wilson 1995; Lamb and Cahill 2008). These disturbances create openings for the establishment of exotic and invasive species that tend to have greater invasion success in soils with increased nitrogen loads (Witkowski 1991; Green and Galatowitsch 2002; Holdredge et al. 2010).

Studies investigating invasion associated with nitrogen deposition in the Great Basin of the western USA have found that nitrogen requirements can be high for invasive grasses due to the rapid growth characteristic of many invasive plants (Evans et al. 2001; Booth et al. 2003). Research suggests that competition for soil nutrients in low nitrogen environments is an abiotic stress that reduces fitness of plants from low $H_{\mathrm{s}}$ populations (Hayes et al. 2005). Recognizing the influence that atmospheric nitrogen deposition has on establishment of low $H_{\mathrm{S}}$ populations provides insight into the processes of invasion and range expansion as the number of environments affected by nitrogen deposition increase.

To investigate how genetic diversity and environmental stress interact to determine colonization success, we studied the invasive perennial bunchgrass Brachypodium sylvaticum (Hudson) Beauv. (slender false brome). The native range of $B$. sylvaticum extends from Northern Europe into Asia and North Africa (Rosenthal et al. 2008). Brachypodium sylvaticum is considered to be aggressively invasive in the Pacific 
Northwest USA, and is classified as a noxious weed in Oregon, USA, where it occupies more than 10,000 hectares of mixed coniferous forest and grasslands (Kaye 2003). Microsatellite marker data have confirmed two independent introductions of $B$. sylvaticum into Corvallis and Eugene, Oregon, USA, as well as multiple introductions of B. sylvaticum from several regions throughout Western Europe (the United Kingdom, Greece, and Spain; (Rosenthal et al. 2008). B. sylvaticum frequently self-fertilizes, allowing a single individual to found a new population. Hybridization among individuals from separate geographic sources has resulted in the spread of novel recombinant genotypes throughout the invaded region (Rosenthal et al. 2008).

Near introduction sites, B. sylvaticum forms expansive monocultures to the exclusion of all other vegetation. In contrast, populations along the range periphery tend to be sparse and are interspersed with other species (Rosenthal et al. 2008).

Brachypodium sylvaticum populations located near the sites of primary colonization exhibit high $H_{\mathrm{S}}$, whereas recently colonized populations possess low $H_{\mathrm{S}}$ (Ramakrishnan et al. 2010). This genetic stratification in invasive populations renders B. sylvaticum an ideal model for the study of invasion dynamics during a species' range expansion.

We assessed the interaction of nitrogen enrichment and population $H_{\mathrm{S}}$ (as a proxy for historical $N_{\mathrm{e}}$ ) on plant performance and for determining outcomes of intra- and interspecific competition in B. sylvaticum. We test for EMID, simulating a less stressful environment by increasing nitrogen levels in plots. We mimicked community dynamics within Oregon sites invaded by B. sylvaticum by growing plants in direct competition with native and introduced grass genotypes originating from the same field locations. The genetic consequences of demographic history were addressed through the collection of 
experimental individuals of $B$ sylvaticum from low or high $H_{\mathrm{s}}$ invasive populations.

Creating "neighborhoods" of competition simulated the arrival of plant species to an open site immediately after disturbance. Individuals of Brachypodium sylvaticum were planted at the center of competition neighborhoods with itself (monoculture neighborhoods) or one of three other bunch grass species (interspecific neighborhoods) in a factorial design with low and high soil nitrogen treatments. Nitrogen levels were carefully chosen to mimic indigenous and elevated nitrogen deposition due to industrialization.

We investigated three primary questions: 1) Is there environmentally- dependent inbreeding depression in invasive populations of B. sylvaticum? 2) Does nitrogen addition result in a release from stress in individuals from low genetic diversity populations of $B$. sylvaticum? and 3) Do nitrogen availability and source population genetic diversity interact to influence plant performance in competition? Competition for nitrogen in a limited environment can result in decreased size and biomass compared to an environment where nitrogen is not limited. Environmentally dependent inbreeding depression would show lower overall fitness in individuals from populations with low $H_{\mathrm{S}}$, with fitness losses exaggerated in the stressful environment (low nitrogen). With EMID individuals from low $H_{\mathrm{S}}$ invasive $B$. sylvaticum populations would experience a release from nutrient stress through nitrogen deposition, and would perform as well as high $H_{\mathrm{s}}$ populations when nitrogen availability is increased. This result would indicate that spread of B. sylvaticum from low genetic diversity populations could be facilitated by human-mediated nitrogen deposition.

\section{Methods}




\section{B. sylvaticum collection and microsatellite genotyping}

The rapidly spreading invasive species $B$. sylvaticum has established populations on the West and East coasts of the USA and Canada (Rosenthal et al. 2008b; Holmes et al. 2010; Miller et al. 2011). It is thought that the United States Department of Agriculture first introduced B. sylvaticum to central Oregon, USA in the 1930's while testing for a productive range grass (Hull 1974; Ramakrishnan et al. 2010). Brachypodium sylvaticum is a diploid, self-compatible, perennial C-3 bunchgrass (Kaye 2003; Ramakrishnan et al. 2010). Brachypodium sylvaticum is nonrhizomatous, although plants frequently spread vegetatively through tillering (Kaye 2003; Ramakrishnan et al. 2010).

We sampled plants from 11 invasive $B$. sylvaticum populations to obtain a range of genetic diversity and inbreeding histories. Seeds collected from sample sites were raised in a greenhouse, and leaf material was dried and stored in silica gel prior to DNA extraction. Genetic diversity was evaluated as within-population heterozygosity using eight to 12 microsatellite markers (SSRs). All microsatellite primers were developed and optimized at Portland State University (Ramakrishnan et al. 2008; Rosenthal et al. 2008) and are deposited at GenBank (Accession nos. EF450748, EF450751, EF450752, EF450754, EF450756, EF450757, EF450759, EF450765). Microsatellite DNA fragment length variation was visualized on an Applied Biosystems 310 automated capillary electrophoresis system with GeneScan 500 ROX as a standard. Allele length variation was scored with Genemapper ${ }^{\mathrm{TM}}$ software (Applied Biosystems). For further details on the microsatellite protocol see Rosenthal et al. (2008). 
For our competition experiment, we selected three perennial, cespitose, nonrhizomatous C-3 bunchgrass species that were consistently found near all $B$. sylvaticum populations examined in the study. Competitor grasses chosen were: Elymus glaucus Buckley (Poaceae; blue wild-rye), Holcus lanatus L. (Poaceae; velvetgrass), and Dactylis glomerata L. (Poaceae; orchardgrass). Holcus lanatus is an escaped cultivar native to Northern Europe and is currently listed as a quarantined invasive species in Washington, California, and Oregon. Dactylis glomerata was first introduced to the USA from Northern Europe in the 1760's and is considered naturalized to the Pacific Northwest. Elymus glaucus is the only native species that was consistently found near $B$. sylvaticum invaded sites.

\section{Experimental Design}

We utilized $H_{\mathrm{S}}$ as a proxy for historical $N_{\mathrm{e}}$ to determine the influence that nutrient deposition and competitor species had on fitness related traits in individuals sourced from invasive B. sylvaticum populations. Due to ethical concerns connected with planting invasive species outdoors for a manipulative experiment, we built six 1 x 1 x $0.8 \mathrm{~m}$ wooden raised beds in which competition neighborhoods were established in a factorial design. Raised beds were located in the hoophouses (polytunnels) at Portland State University, Portland, OR, USA. The hoophouse was a polyethylene-covered tunnel semicircular with minimal environmental control. Fans provided air circulation and cooling when temperatures rose above $23^{\circ} \mathrm{C}$ in the summer while hoophouses were unheated throughout the winter in order to better mimic ambient temperatures.

We designated three raised beds as high nitrogen (nitrogen addition) and three raised beds as low nitrogen (no nitrogen addition). Light conditions within the hoophouse 
were similar to light levels at B. sylvaticum sites in the Willamette Valley, Oregon, USA. Soil used was a sandy loam mixture consisting of 5\% clay, $45 \%$ sand and $50 \%$ silt containing nitrogen levels similar to that of soil at field sites (Field site average: $10 \mathrm{ppm}$ $\mathrm{NO}_{3}{ }^{-}-\mathrm{N}, 2$ ppm $\mathrm{NH}_{4}{ }^{+}-\mathrm{N}, \mathrm{pH}$ 6.5; Sandy loam: 16 ppm $\mathrm{NO}_{3}{ }^{-}-\mathrm{N}, 2$ ppm $\mathrm{NH}_{4}{ }^{+}-\mathrm{N}, \mathrm{pH}$ 6.7).

Soil from all sites used as source populations was collected, mixed, and spread approximately three centimeters deep over the sandy loam mixture to serve as a microbial inoculum and to better simulate field conditions.

Seeds of B. sylvaticum, E. glaucus, D. glomerata, and H. lanatus species were collected from 11 Oregon, USA sites located at least $10 \mathrm{~km}$ apart in October 2008 (see Table 1 for site locations). Seeds were collected from plants located at least 10 meters apart. The location of the experimental site at the Portland State University hoophouses was $120 \mathrm{~km}$ from the nearest collection site. In February 2009, seeds from all species were cold-stratified at $4^{\circ} \mathrm{C}$ for 28 days prior to planting in order to mimic winter dormancy and achieve coordinated germination. After cold stratification, seeds were placed on water-moistened germination paper for one week. Seeds that had germinated at the end of this period with a coleoptile less than $1 \mathrm{~cm}$ were planted into competition neighborhoods within raised beds in April 2009.

In each bed, germinated individuals were planted within a matrix of $6436 \mathrm{~cm}^{2}$ square competition neighborhoods consisting of five individuals (Fig. A.B1). Neighborhoods always consisted of individuals originating from the same source population and were randomized within the raised beds. At the center of each neighborhood we placed a $B$. sylvaticum individual as the focal target plant for which we measured competitive effects. Focal seedlings originated from different maternal plants 
collected from each population site. In these neighborhoods, each target individual was surrounded at the four corners of the square by E. glaucus, H. lanatus, D. glomerata (interspecific competition), or B. sylvaticum (monoculture, intraspecific competition; see Fig. A.B1 for competition design). Focal B. sylvaticum seedlings were spaced $10 \mathrm{~cm}$ apart in the center of each neighborhood, with competitor seedlings located at the four corners of each square. Species combinations were planted in approximately equal numbers for all source populations across raised beds $(\mathrm{n}=\sim 10$ neighborhoods per source population $\mathrm{x}$ competitor combination in each nitrogen treatment). Although the design of this experiment resulted in plants interacting with individuals beyond their immediate neighborhood, we do not consider those effects in this study. Nitrogen was applied to the high nitrogen beds in low level increments to simulate the natural deposition rate in central Oregon of $16 \mathrm{~kg} * \mathrm{~N} / \mathrm{ha}^{*} \mathrm{yr}$ (Galloway et al. 2008). This number was scaled down to account for the size of the raised beds and applied weekly as $30.8 \mathrm{mg} \mathrm{NH} \mathrm{N}_{4} \mathrm{NO}_{3} / \mathrm{m}^{2}$, dissolved in water, applied during the regular watering schedule. All raised beds were given $10 \mathrm{~L}$ of water every other day.

\section{Variables Measured}

Measurements were made across three summer growing seasons to estimate plant size and reproduction as surrogates for fitness. Plant height and tiller number of the $B$. sylvaticum target plants were chosen as fitness characters as these capture competitive ability for light (height) and space (tiller number). Because B. sylvaticum exhibits growth vertically and through tiller production, we estimated plant size defined as height and shoot number prior to harvest. Plants were harvested 24 months after planting and shoot biomass was obtained by placing plants in convection drying ovens $\left(70^{\circ} \mathrm{C}\right)$ until a 
constant mass was achieved (minimum of 48 hours). We were not able to acquire root biomass as roots become tangled after long growth periods, making them difficult to pull apart without significant loss.

\section{Relative Competition Index}

We calculated coefficients of relative competition intensity (RCI; Campbell and Grime 1992; Weigelt and Jolliffe 2003) to quantify the intensity of inter- and intraspecific competition for each focal B. sylvaticum individual per nitrogen treatment and source population. The $R C I$ has defined limits $[-1,+1]$ and is symmetrical around zero; a value from 0 to +1 indicate that species performs better in mixture than in monoculture, experiencing reducing competition with interspecific neighbors; values from 0 to -1 indicate no competition between species (Williams and McCarthy 2001). The RCI is calculated by dividing the difference of the biomass of the B. sylvaticum individuals grown in monoculture $\left(B_{\mathrm{MONO}}\right)$ and $B$. sylvaticum focal individuals grown with interspecific competitors ( $\left.B_{\mathrm{MIX}}\right)$ by the biomass of $B$. sylvaticum focal individuals grown in monoculture $\left(\left(\left(B_{\mathrm{MONO}}-B_{\mathrm{MIX}}\right) /\left(B_{\mathrm{MONO}}\right)\right) \times 100\right)$.

We used ANOVA to determine the effects of $H_{\mathrm{S}}$ and nitrogen application on $R C I$ (R Core Development Team 2014). Explanatory variables in the model were $H_{\mathrm{S}}$, nitrogen, and their interaction; the response variable was $R C I$.

\section{Statistical Analyses}

We evaluated the interactions between nitrogen enrichment, source population $H_{\mathrm{S}}$, and competitor species for their effects on final plant size and shoot biomass. Our study was designed to have six raised beds with two different treatments, and we analyzed the raised beds as random factors, with individual plants nested within the beds. The 
response variables plant size and shoot biomass were log transformed to fit assumptions of normality for ANCOVA. Although plants are interacting with individuals beyond their immediate neighborhood, random positioning of neighbor treatments ensures that any effects of these interactions are not systematically applied to particular treatments. We recognize that samples from a population are not all genetically equivalent, but in our statistical models we assume that $H_{\mathrm{S}}$ is descriptive of the common history of a population. Data was analyzed with mixed model ANCOVAs using the lme command of the nlme package in R: Statistical Software (R Core Development Team 2014; Pinheiro J 2015). Our analyses were also performed categorically with $H_{\mathrm{S}}$ and substituting $F_{\text {IS }}$ for $H_{\mathrm{S}}$, these results can be found in Appendix B.

\section{Results}

Nitrogen application and $H_{\mathrm{S}} \mathrm{x}$ Nitrogen interaction were significant for shoot biomass and final size. Other model terms and interactions were not significant (Table 2). Population $H_{S}$ x Nitrogen Interaction

Population genetic diversity ranged from almost completely homozygous $\left(H_{\mathrm{S}}=0\right)$ to highly heterozygous $\left(H_{\mathrm{S}}=0.392\right.$; Table 3.1; Rosenthal et al. 2008). Nitrogen addition alone had a positive effect on final size $\left(\mathrm{F}_{1,4}=37.68, \mathrm{p}=0.0036\right)$ and shoot biomass $\left(\mathrm{F}_{1,4}\right.$ $=16.26, \mathrm{p}=0.0157)$. At low $H_{\mathrm{S}}$, nitrogen addition displayed a positive effect on final size $\left(F_{1,220}=6.933, p=0.0091\right)$ and shoot biomass $\left(F_{1,230}=6.06, p=0.0146 ;\right.$ Figure 3.2 $) . A$ categorical presentation of this data can be found in Appendix B, Fig. A.B2. From categorical data, it was seen that plants from low $H_{\mathrm{S}}$ populations in the high nitrogen treatment had a $78 \%$ increase in size and a $12 \%$ increase in shoot biomass compared to individuals from low $H_{\mathrm{S}}$ populations grown without nitrogen addition. 


\section{Relative Competition Index}

Relative competition index coefficients indicate that although there is a slight downward trend in the intensity of competition with increasing $H_{\mathrm{S}}$, this relationship was not significant in our experiment (Fig. 3.3). Means of the coefficient of RCI are negative for plants from high nitrogen neighborhoods (mean $=-0.054 \pm 0.066 \mathrm{SE}$ ) and positive for plants from low nitrogen neighborhoods (mean $=0.004 \pm 0.117 \mathrm{SE})$. Results from ANOVA were not significant for nitrogen application, $H_{S}$, their interaction term. This indicates similar performance of individuals from low and high $H_{S}$ populations.

\section{Discussion}

The primary aim of this study was to investigate the interaction of abiotic stress and population genetic diversity $\left(H_{\mathrm{S}}\right)$ on performance of an invasive species. We simulated community dynamics of species arrival at a disturbed site to determine how $H_{\mathrm{S}}$, a genetic factor that is a function of historical $N_{e}$, influences performance of the invasive plant B. sylvaticum. Nitrogen addition resulted in plants from low $H_{S}$ populations displaying fitness-related trait values similar to those exhibited by individuals from high $H_{S}$ populations (Fig. 3.2). Results show ecological mitigation of inbreeding depression (EMID), suggesting that bottlenecks in the invasive range facilitated local adaptation of low genetic diversity populations to increased nitrogen availability. This argument is strengthed by the result that individuals from populations with high $H_{S}$ values had no difference in vigor across nitrogen treatments.

The finding that nitrogen application significantly increased growth of individuals from low $H_{S}$ populations provides insight into the success of invasive species with small population sizes and limited genetic diversity. Our results are consistent with other 
studies finding fitness declines for plants from low genetic diversity populations under stressful conditions, however, these studies assume constant reductions in fitness for homozygous individuals (Damgaard and Loeschcke 1994; Cheptou et al. 2001; Fox and Reed 2011; Bijlsma and Loeschcke 2012). Equal performace of plants from low and high genetic diversity populations in response to environmental conditions has not previously been noted as a mechanism of adaptation in invasive species.

The ability of invasive individuals from low genetic diversity populations to more effectively respond to nitrogen application indicates EMID, and implies that local adaptation towards increased utilization of nutrients is specific to more homozygous populations. Local adaptation is the evolution of traits that result in a fitness advantage in conditions of the local habitat, regardless of the consequences of these traits in other environments (Williams 1966). In high $H_{\mathrm{S}}$ populations, selective processes can be driven by temporal ecological variability, shielding individuals from the effects of selection towards specific environments. This is likely why the release from nitrogen stress had no effect on fitness of B. sylvaticum individuals from high $H_{\mathrm{S}}$ populations. EMID may be hindered by frequent gene flow and higher connectivity of invasive populations.

Selection for plasticity of traits associated with nutrient uptake could lead to EMID in low $H_{\mathrm{S}}$ populations through adaptive trait responses to increased resource availability. In a variable environment, plasticity can increase the ability of a species to survive by allowing individuals to modify morphological or physiological traits to values optimal to the habitat. In a fairly constant environment, one phenotype may consistently have greater fitness than other phenotypes, resulting in selection towards a single, optimal 
phenotype. In low $H_{\mathrm{S}}$ populations of $B$. sylvaticum selection may have favored plasticity for improved uptake of nitrogen.

Genetic purging, strong selection on deleterious alleles that accumulate as a result of inbreeding and increasing homozygosity can also lead to EMID. Environmentally determined genetic purging could improve fitness responses to increased resource availability, but not alleviate fitness deficits under more stressful conditions. Evidence for genetic purging has been found in invasive populations of B. sylvaticum (Marchini et. al in press). Reduced inbreeding depression in offspring of individuals from low $H_{\mathrm{s}}$ populations compared to progeny from high $H_{\mathrm{s}}$ populations was found for $B$. sylvaticum plants from in invasive range (Marchini et. al in press). Because genetic purging may be dependent on environmental stress, individuals can experience strong fitness losses when subjected to environmental fluctuations such as reduced nutrient availability.

Inbreeding is thought to interfere with the ability of individuals to successfully grow under stressful and low nutrient conditions (Fox and Reed 2011). As nitrogen deposition is common, especially in the disturbed habitats that are easily colonized by introduced species (Mooney and Hobbs 2000), nutrient addition is likely a consistent selective force throughout the invasive range of many species. Local adaptation to increased soil fertility in the introduced range has been previously documented as a likely facilitator of invasion success for the invasive grass Bromus inermis (Sherrand and Maherli 2012). Nitrogen fertilization improves plant performance and the chance that low $H_{S}$ populations of $B$. sylvaticum will thrive and serve as a source for subsequent establishment, allowing invasive plants to overcome the limitations imposed by small $N_{e}$ and demographic stochasticity. 
Interestingly, there was a weak overall effect of competition on B. sylvaticum performance. Under the conditions of this experiment, the abiotic conditions such as nitrogen availability had a larger effect on plant fitness than on performance in response to competition. We did not find any significant effect of $H_{\mathrm{S}}$ or nitrogen application on $R C I$. This is unexpected, as inbreeding depression and homozygosity are thought to decrease plant performance in competition (Donohue 1998; Vellend 2006; Cheptou and Donohue 2011), although is in line with results that low $H_{\mathrm{S}}$ populations have become adapted to the invasive environment. Low genetic diversity does not influence performance, indicating that competition is not any more of a barrier to low $H_{\mathrm{S}}$ populations that are newly establishing in the invasive range than it is to high $H_{\mathrm{S}}$ populations.

Our study provides evidence that nitrogen deposition leads to ecological mitigation of environmentally determined inbreeding depression and can facilitate the establishment of low $H_{\mathrm{S}}$ populations of invasive species. The loss of genetic diversity in newly- established populations is expected to slow population growth and consequently the rate of range expansion; however the opposite was found for plants in nitrogen-added plots. These results provide evidence of local adaptation in populations with low genetic diversity, in contrast to populations with high genetic diversity. Further study may reveal whether the superior response of low $H_{\mathrm{S}}$ populations to increased nitrogen availability is the outcome of improved plasticity for traits associated with $\mathrm{N}$ uptake or to environmentally-dependent effects of genetic purging during invasion. Elevated nutrient deposition may be allowing populations of $B$. sylvaticum to overcome genetic 
establishment barriers, enhancing rates of range expansion and raising the probability that invasive species will remain and thrive. 


\section{Tables and Figures}

Table 3.1 Location information, $H_{S}$, and $H_{O}$ values for $B$. sylvaticum populations.

\begin{tabular}{llllll}
\hline Site Code & Latitude & Longitude & $\mathrm{N}$ & $H_{S}$ & $H_{O}$ \\
\hline C1 & $44^{\circ} 39^{\prime} 35^{\prime \prime}$ & $-124^{\circ} 45^{\prime} 41^{\prime \prime}$ & 24 & 0.26 & 0.017 \\
E1 & $43^{\circ} 57^{\prime} 35^{\prime \prime}$ & $-123^{\circ} 15^{\prime} 49^{\prime \prime}$ & 34 & 0.276 & 0.085 \\
E4 & $44^{\circ} 0^{\prime} 48^{\prime \prime}$ & $-123^{\circ} 7^{\prime} 30^{\prime \prime}$ & 14 & 0.042 & 0.036 \\
E6 & $43^{\circ} 59^{\prime} 47^{\prime \prime}$ & $-123^{\circ} 12^{\prime} 3^{\prime \prime}$ & 24 & 0.37 & 0.073 \\
E7 & $43^{\circ} 58^{\prime} 29^{\prime \prime}$ & $-123^{\circ} 21^{\prime} 13^{\prime \prime}$ & 21 & 0.097 & 0.043 \\
E9 & $43^{\circ} 58^{\prime} 26^{\prime \prime}$ & $-123^{\circ} 7^{\prime} 36^{\prime \prime}$ & 24 & 0.392 & 0.176 \\
M16 & $44^{\circ} 22^{\prime} 19^{\prime \prime}$ & $-123^{\circ} 22^{\prime} 45^{\prime \prime}$ & 24 & 0.297 & 0.19 \\
M3 & $44^{\circ} 23^{\prime} 45^{\prime \prime}$ & $-122^{\circ} 28^{\prime} 13^{\prime \prime}$ & 21 & 0 & 0 \\
M5 & $44^{\circ} 24^{\prime} 30^{\prime \prime}$ & $-122^{\circ} 36^{\prime} 22^{\prime \prime}$ & 21 & 0.2 & 0.05 \\
M7 & $44^{\circ} 24^{\prime} 7^{\prime \prime}$ & $-122^{\circ} 39^{\prime} 59^{\prime \prime}$ & 21 & 0.34 & 0.14 \\
S1 & $44^{\circ} 45^{\prime} 14^{\prime \prime}$ & $-123^{\circ} 28^{\prime} 56^{\prime \prime}$ & 29 & 0.063 & 0.086 \\
\hline
\end{tabular}


Table 3.2 Statistical results presented as F-values from mixed models testing the effects of $H_{S}$, nitrogen fertilization (N), competitor species (S), and their interaction terms on final size and shoot biomass of B. sylvaticum focal plants.

\begin{tabular}{cccc}
\hline Variable & df & Final Size & Shoot Biomass \\
\hline$H_{\mathrm{S}}$ & 1 & 0.39 & 3.39 \\
$\mathrm{~N}$ & 1 & $\mathbf{3 7 . 6 8}^{* *}$ & $\mathbf{1 6 . 2 6}^{*}$ \\
$\mathrm{~S}$ & 3 & 0.68 & 0.61 \\
$H_{\mathrm{S}} \times \mathrm{N}$ & 1 & $\mathbf{6 . 9 3}^{* *}$ & $\mathbf{6 . 0 6}^{*}$ \\
$H_{\mathrm{S}} \times \mathrm{S}$ & 3 & 1.37 & 0.86 \\
$\mathrm{~N} \times \mathrm{S}$ & 3 & 0.56 & 1.81 \\
$H_{\mathrm{S}} \times \mathrm{N} \times \mathrm{S}$ & 3 & 0.92 & 0.37 \\
\hline
\end{tabular}

Bold values indicate statistical significance, $* \mathrm{P}<0.05, * * \mathrm{P}<0.01$. 
Figure 3.1 Environmentally dependent-inbreeding depression (EDID) and ecological mitigation of inbreeding depression in individuals in nutrient-rich and nutrient-poor conditions. Solid lines show predicted fitness for individuals from low genetic diversity (dark blue) and high genetic diversity (gray) populations. $(A)$ Inbreeding depression results in an overall reduction in fitness for inbred individuals, EDID results in increased expression of inbreeding depression in the nutrient-poor environment (Cheptou 2011). $(B)$ EMID results in higher fitness of individuals from low genetic diversity populations in the less stressful nutrient-rich environment. Increases in fitness are not seen for individuals from high genetic diversity populations where selective processes did not occur.
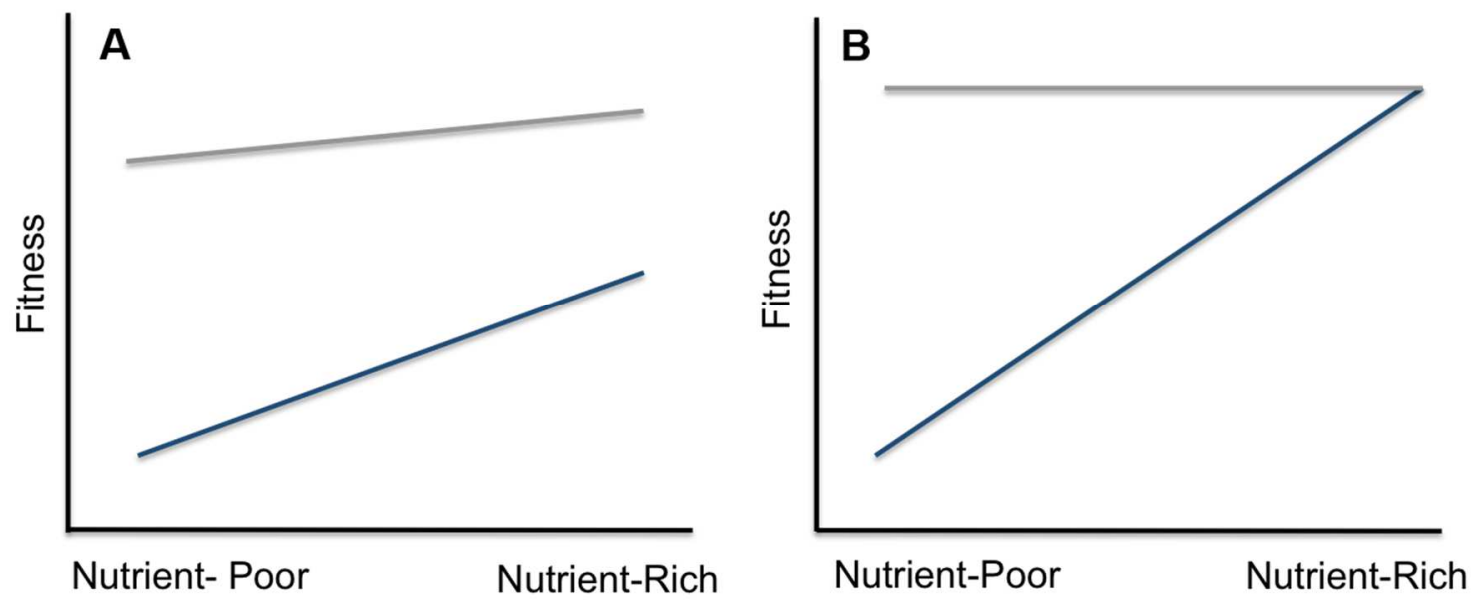
Figure 3.2 $(A)$ Nitrogen $\mathrm{x} H_{\mathrm{S}}$ effects on final size (height*shoot number) and $(B)$ Nitrogen $\mathrm{x} H_{\mathrm{S}}$ effects on shoot biomass of $B$. sylvaticum focal plants. Orange dots represent plants in high nitrogen neighborhoods; blue dots represent plants in low nitrogen neighborhoods. Regression lines indicate $H_{\mathrm{S}}$ by final size and shoot biomass interactions in high nitrogen (orange lines) and low nitrogen (blue lines) neighborhoods. Grey areas represent $95 \%$ confidence intervals. Increased $\mathrm{N}$ resulted in significant differences in performance for individuals from low $H_{\mathrm{S}}$ populations, but this difference declined with increasing $H_{\mathrm{S}}$, with no difference between low and high $\mathrm{N}$ treatments at the highest $H_{\mathrm{S}}$ values (see Appendix B1).
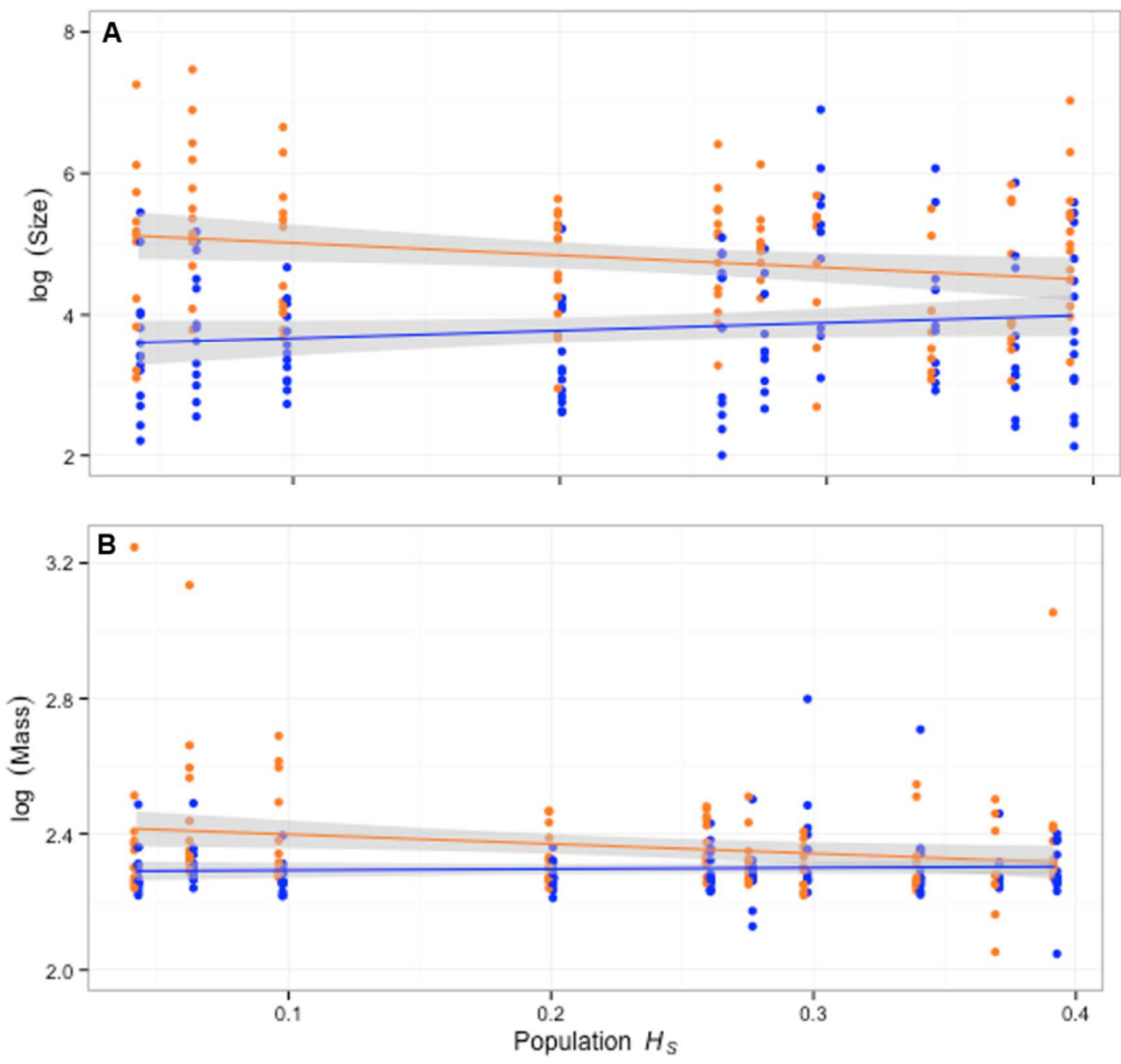
Figure 3.3 Relative competition index coefficient (RCI) values for each per high and low nitrogen treatment for each source population $H_{\mathrm{S}}$. Orange dots represent plants in high nitrogen neighborhoods; blue dots represent plants in low nitrogen neighborhoods. Regression lines indicate $H_{\mathrm{S}}$ in high nitrogen (orange lines) and low nitrogen (blue lines) neighborhoods. RCI values greater than 0 indicate a suppressive effect of interspecific competitors; values below zero indicate possible facilitative effects in monoculture. Population $H_{\mathrm{S}}$ and $\mathrm{N}$ did not result in significant differences in plant performance.

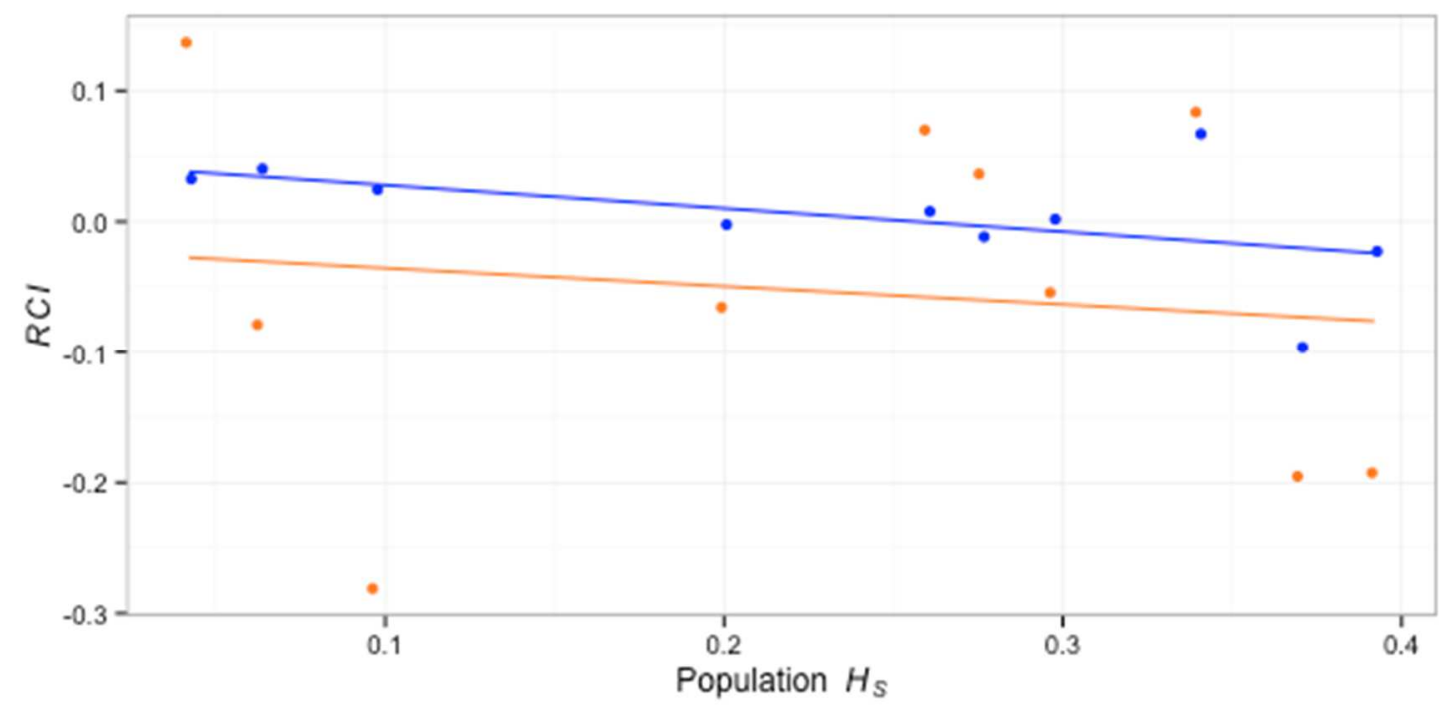




\section{Chapter 4}

Selective differentiation during the colonization and establishment of a newly invasive species

\section{Summary:}

- The potential for rapid evolution in invasive species makes them useful for studying adaptive responses of populations to novel environments. However, phenotypic divergence during invasion is not necessarily due to selection, but may be a product of neutral processes resulting from population bottlenecks during colonization and range expansion.

- We investigated phenotypic adaptation during the establishment and range expansion of the invasive bunchgrass, slender false brome (Brachypodium sylvaticum; Poaceae). We utilized a novel approach to make robust comparisons of functional traits using unique alleles to determine the genetic probability of contribution from native source regions. These probabilities were used as weights in $Q_{\mathrm{ST}}-F_{\mathrm{ST}}$ comparisons for twelve functional traits associated with drought stress in the introduced range.

- Our results indicate phenotypic divergence greater than neutral expectations in five traits between native and invasive populations, indicating selective divergence had occurred during invasive species establishment. Results show that the majority of divergence in B. sylvaticum occurred after introduction to the novel environment, but prior to invasive range expansion. 
- This study provides evidence for adaptive genetic differentiation during the establishment of an invasive species, while also describing a robust method for detection of selective processes after species introduction to a novel environment.

\section{Introduction}

The potential for rapid evolution in invasive species makes them a useful tool for studying adaptive responses of populations introduced to novel environments (Prentis et al. 2008; Sultan et al. 2013; Franks and Munshi-South 2014). While evolutionary processes during invasion are evidenced by divergence in fitness-related traits in invasive populations (Bossdorf et al. 2005; Olivieri 2009; Wilson et al. 2009), investigating the mechanisms underlying this differentiation can be difficult (Keller and Taylor 2008). Divergence of an invasive species is not necessarily the result of selection but may be a product of selectively neutral processes (i.e., genetic drift) resulting from population bottlenecks during introduction and establishment. Disentangling the influence of genetic drift and selection on phenotypic divergence during establishment after long-distance dispersal provides much needed knowledge on the potential for adaptation to novel environments to promote range expansion.

There are several phases of a species invasion where selection can occur (Prentis et al. 2008), but these may be counteracted by population bottlenecks. Genetic bottlenecks are common after propagules are first transported to a novel environment, even though successfully invasive species are often the product of multiple introductions that reduce the effects of bottlenecks and increase evolutionary potential (Lavergne and Molofsky 2007; Rosenthal et al. 2008). Ease of establishment of these colonizing 
individuals is determined by phenotypic plasticity and the degree of pre-adaptation to novel biotic and abiotic environmental conditions; even moderately pre-adapted species often incur an initial lag phase of slow demographic growth after which vigorous population expansion may occur (Aikio et al. 2010; Lenda et al. 2012). Genetic drift or selection during the lag phase (pre-invasion differentiation), or during invasive range expansion following introduction (post-invasion differentiation), can result in trait divergence between native and invasive populations. Bottlenecks and selection can occur during establishment or the subsequent range expansion of an invasive species, and the ultimate success of invasion may be dependent on evolutionary processes occurring at both stages.

To differentiate the influence of selection and genetic drift on variation in phenotypic traits, $Q_{\mathrm{ST}}$, a measure of quantitative genetic differentiation, can be compared to $F_{\mathrm{ST}}$, which measures population differentiation for neutral molecular loci (Wright 1931; Lande 1992; Spitze 1993; Whitlock 2008; Leinonen et al. 2013). Because historical processes such as genetic drift affect neutral markers across a genome, $Q_{\mathrm{ST}}$ and $F_{\mathrm{ST}}$ are expected to be approximately equal if trait differentiation is the result of neutral evolution. Divergence due to natural selection in the novel environment increases the variance (differences among populations) in $Q_{\mathrm{ST}}$ relative to $F_{\mathrm{ST}}\left(Q_{\mathrm{ST}}>F_{\mathrm{ST}}\right)$. A reduction in $Q_{\mathrm{ST}}$ relative to $F_{\mathrm{ST}}$ is indicative of homogenizing selection (e.g., due to similar habitat conditions), where phenotypic differentiation among populations is reduced compared to the neutral expectation $\left(Q_{\mathrm{ST}}<F_{\mathrm{ST}}\right)$. Previous studies have found phenotypic divergence of invasive species in invaded regions but have not always successfully differentiated the 
effects of selection from the effects of drift (e.g., Burns 2004; Bossdorf et al. 2005; Leishman et al. 2007).

Detection of adaptive divergence in invasive populations can be problematic if native source regions are unknown. Many invasive species possess broad native distributions, and only a subsample of the populations may have contributed to an invasion. If native populations used for $Q_{\mathrm{ST}}-F_{\mathrm{ST}}$ comparisons are all treated as equally contributing to an invasion, selection in the invaded range may be falsely inferred because the native trait values are not representative of the populations that established the invasion. Here we use unique genetic profiles based on twelve microsatellite loci among native populations to identify the populations contributing to the invasion. We use these probabilities of contribution to weight $Q_{\mathrm{ST}}$ and $F_{\mathrm{ST}}$ calculations, providing a robust test of divergence by using values of quantitative traits that were most likely present upon original establishment of the species in the invasive habitat.

To study adaptive divergence during establishment and range expansion, we utilized the invasive species Brachypodium sylvaticum (Hudson) Beauv. (slender false brome), a newly invasive perennial C-3 bunchgrass in the Pacific Northwest region of the United States (Rosenthal et al. 2008; Roy 2010). Invasive populations of B. sylvaticum are concentrated in the states of Oregon and California, USA. The native range of $B$. sylvaticum extends across Europe, North Africa, and parts of Asia (Catalan and Olmstead 2000). It is thought that B. sylvaticum was first introduced into Eugene, Oregon, USA, and Corvallis, OR, USA during rangeland testing experiments utilizing combinations of plants from populations across the species' native range (Rosenthal et al. 2008). Rosenthal et al. (2008) utilized microsatellites (SSRs) to determine source regions 
contributing to the introduction, and found unique combinations of alleles in invasive individuals that were not present in native plants indicating that invasive individuals consist of recombinant intra-specific hybrids (Rosenthal et al. 2008). After its introduction into Oregon in the 1930's, B. sylvaticum experienced a lag period of about 50 years and did not undergo invasive range expansion until the late 1980s (Rosenthal et al. 2008). Additionally, seed stock from native accessions presumably utilized for initial rangeland plantings is still maintained and available from the Western Regional Plant Introduction Station in Pullman, Washington, USA. The combination of available information from previous studies on B. sylvaticum's source regions, as well as access to the seed stock representing the probable source of invasion renders B. sylvaticum an outstanding system for comparative studies and investigations of divergence during invasion.

Detection of phenotypic divergence in the invasive region is dependent on the traits selected for quantification. Environmental stressors such as climate, and precipitation in particular, can be a significant evolutionary force for plants. B. sylvaticum has a wide geographic native range with variable precipitation levels during the summer growing season (May- September) including such areas as the Southern United Kingdom, a region with a wet summer growing season, and Northern Greece, a region with a dry summer growing season. We compared the summer aridity of central Oregon, USA, the primary site of invasion of B. sylvaticum in the USA to the summer aridity values of native source regions identified by Rosenthal et al. (2008) and found that, on average, the long drought occurring during the Oregon summer months results in more arid conditions than what is found in native regions. 
To understand potential responses to the more arid conditions in the invasive range we quantified a wide range of physiological and anatomical traits associated with plant performance under water-limited conditions. Lack of precipitation in the invasive range can result in drought stress, a plant's response to a period of time of below average precipitation that can result in reduced productivity. The ability of plants to function during drought is related to a combination of physiological and morphological traits promoting drought tolerance or resistance (Grime 2001). This is particularly the case for B. sylvaticum because flowering and seed set occur in late summer during the driest part of the growing season. Traits promoting drought tolerance can vary across individuals of the same species and even across the lifespan of an individual. Maintenance of cell turgor and normal tissue water potentials $\left(\Psi_{\mathrm{W}}\right)$ during drought requires reducing water losses through exposed surfaces by rolling or folding in leaves or closing stomata (Verslues and Zhu 2005). Long-term water deficit can result in reduced specific leaf area (thicker leaves) or smaller, more numerous stomata to limit transpirational and surface water loss, or alteration of xylem morphology and cell wall stiffness (Meinzer and Grantz 1990; Tyree and Zimmermann 2002; Verslues and Zhu 2005; Tombesi et al. 2010; Aroca et al. 2012).

We used a combination of common garden studies examining physiological and morphological traits related to drought tolerance and population genetic analyses to assess patterns of phenotypic divergence during establishment and initial range expansion of invasive B. sylvaticum populations in Oregon. Our study tests the hypothesis that selection during establishment resulted in trait divergence throughout B. sylvaticum's invasion into the Pacific Northwest, USA. The molecular evidence of hybridization found 
by Rosenthal et al. (2008) supports this hypothesis, as multiple introductions first increased genetic diversity of invasive populations, increasing evolutionary potential and providing variation for selection on intra-specific hybrids during the establishment phase of invasion, aiding the range expansion and invasion of $B$. sylvaticum into the Pacific Northwest USA.

In this study, we addressed three main questions: (1) Does divergence in quantitative traits exist between native and invasive populations of B. sylvaticum in functional traits related to water availability? (2) If present, is divergence in quantitative traits the result of genetic drift or selection in the invasive region? and, (3) At what point during the invasion of $B$. sylvaticum did divergence in quantitative traits occur; during establishment (pre-invasion) or after invasive range expansion (post-invasion)? We obtained and utilized accessions of seeds presumably used for the original rangeland planting experiment that established B. sylvaticum in Oregon. Data were collected on twelve functional traits related to water stress in individuals from native and invasive populations grown in the greenhouse. When comparing $Q_{\mathrm{ST}}$ and $F_{\mathrm{ST}}$ between native and invasive regions we integrated the probability of contribution from native populations to the invasive range as weights. We predicted that selection during establishment (preinvasion) would exhibit significantly greater $Q_{\mathrm{ST}}$ vs. $F_{\mathrm{ST}}$ values for comparisons between invasive and native regions, and selection in different habitats occurring during range expansion (post-invasion) would result in significant $Q_{\mathrm{ST}}-F_{\mathrm{ST}}$ values among populations in the invasive range.

\section{Materials and Methods}

Seed Collection and Genetic Analysis 
For this study, we utilized plants from the same populations that were sampled for microsatellite variation by Rosenthal et al. (2008). Plant seeds were collected from 11 invasive populations located in Oregon, USA and 21 native populations located throughout Europe, North Africa, and the Middle East (Table 4.1). Brachypodium sylvaticum (Hudson) Beauv. was most likely first introduced to Oregon, USA during planting experiments in the cities of Eugene and Corvallis conducted by the Western Regional Plant Introduction Station (Western Regional Plant Introduction Station, Pullman, WA, USA). Seeds from additional European populations were collected by individuals from Portland State University (M. Poyourow and M.B.C.) and from European institutions (Pilar Catalan, University of Zaragoza; Michael Ristow, University of Potsdam), or were provided by the Millennium Seed Bank Project (Kew, Surrey, UK; Table 4.1). At least eight individuals were analyzed from each of the populations in the native range with the exception of Germany $(\mathrm{N}=6)$, Slovakia $(\mathrm{N}=2)$, and $\operatorname{Iran}(\mathrm{N}=7)$; between 20 and 30 individuals were analyzed for each population from the invasive range in Oregon (Table 4.1). Plants were genotyped at eight microsatellite loci with primers developed and optimized at Portland State University (Ramakrishnan et al. 2008) and deposited at GenBank (Accession nos EF450748, EF450751, EF450752, EF450754, EF450756, EF450757, EF450759, EF450765). As the detailed information for the microsatellite survey is presented elsewhere (Rosenthal et al. 2008), we will only discuss the additional analyses performed for the present study in the main manuscript (see Methods AC.1 for microsatellite survey methods).

Brachypodium sylvaticum is widely distributed across a range of environments, including semi-arid deserts, dry-summer temperate Mediterranean, and Oceanic climates. 
The aridity index $A-$ a numerical index of the degree of dryness at a location where smaller values indicate a more arid environment- was calculated from interpolated climatic data, (including 30-year monthly temperature averages and total monthly precipitation), for invasive and source regions of B. sylvaticum using the method of Malmstrom (1969). Climatic data for Oregon, USA populations were obtained from the Prism Group, Oregon State University (PRISM Group 2004); climatic data for native source populations located throughout Europe, the Middle East, and Asia were obtained using WorldClim (Hijmans et al. 2005) and United State Geological Climate Survey (USGS) climate data (Hearn et al. 2003). We found that invasive populations in the Willamette Valley of Oregon, USA, the center of the B. sylvaticum invasion had an average summer aridity of 0.71 , while regions near the native source populations in Northern Greece had an average summer aridity of 0.42 , and native source populations in the Southern UK had an average summer aridity of 0.90 (see Methods A.C2, Fig. A.C1 for details).

We determined the probability of contribution from different native source populations to invasive populations. Assignment tests for the probability of genetic contribution of native to invasive populations were performed in GeneClass 2 using twelve microsatellite loci with the Rannala and Mountain (1997) assignment method of identifying immigrants based on their multilocus genotypes (Rannala and Mountain 1997; Piry et al. 2004). The assignment probabilities were pooled across invasive populations to create an average probability of genetic contribution of each native population to the invasive range. These probabilities were used as weights in ANOVA and $Q_{\mathrm{ST}}-F_{\mathrm{ST}}$ comparisons described below, allowing for a more accurate assessment of 
differentiation in the invasive range than if all native populations were pooled for comparative analysis.

\section{Common Garden Studies}

Two common garden studies were conducted to measure of functional traits related to water availability in B. sylvaticum. The first study involved the creation of pressure-volume curves measuring the relationship between water potential $(\Psi)$ and relative water content in dehydrating leaves. Pressure-volume curves summarize leaf responses to water deficit and allow calculation of key leaf parameters related to drought tolerance (Tyree and Hammel 1972; Bartlett et al. 2012). The second common garden study allowed measurement of leaf morphological traits that may be phenotypically variable in response to water availability of the environment. Expected trait responses to water deficit are found in Table 4.2.

\section{Common garden study 1: Pressure Volume Curves}

Ten individuals from each of thirty-six native and invasive populations of $B$. sylvaticum were grown from seed in the Portland State University (PSU) glasshouse in June 2011. Seeds of invasive populations were collected from field sites in Oregon, USA. Seeds of native populations were obtained from the Western Plant Introduction Station (Pullman, WA, USA) and the Millennial Seed Bank Project (Kew, Surrey, UK; Table 4.1). Plants were considered fully grown five months after planting. Three to six individuals per population were randomly selected for creation of pressure-volume curves during the period from December 2011 to April 2012. Prior to processing, leaves were cut from plants submerged in water, leaves were then left in tap water for 24 hours in the dark to allow full hydration. The mean initial water potential of all leaves used was -0.90 
$\mathrm{MPa}$. Leaves were placed in a $60^{\circ} \mathrm{C}$ drying oven for a minimum of 48 hours then weighed to determine dry mass.

Water potential measurements and creation of pressure volume curves were performed following the procedure of Turner (1981) using a Scholander Pressure Chamber (Model 600, PMS Instrument Company, Albany, OR, USA). Leaves were weighed to $0.001 \mathrm{~g}$ immediately before and after water potential measurements. Measurements were considered complete when a water potential of -2.5 MPa was obtained. We derived six physiological parameters from graphical analysis of pressurevolume curves: (1) Turgor loss point ( $\left.\Psi_{\mathrm{TLP}}, \mathrm{MPa}\right)$, the point during dehydration when leaf cells become flaccid and lose turgor; (2) Relative water content at turgor loss point $\left(R W C_{\mathrm{TLP}}, \%\right)$, the leaf hydration at which cells become flaccid; (3) Osmotic potential at full hydration $\left(\pi_{\mathrm{o}}, \mathrm{MPa}\right)$, the solute concentration in plant cells; (4) Cell modulus of elasticity at full turgor ( $\varepsilon, \mathrm{MPa})$, plant cell wall stiffness describing the strain that occurs in response to water stress; (5) Relative capacitance at full turgor $\left(C_{\mathrm{FT}}, \mathrm{MPa}^{-1}\right)$, the water storage capacity for leaves at full turgor; and (6) Relative capacitance at turgor loss point $\left(C_{\mathrm{TLP}}, \mathrm{MPa}^{-1}\right)$, the water storage capacity for leaves at turgor loss point (Tyree and Hammel 1972; Sack et al. 2003; Bartlett et al. 2012; Table 2).

The inverse of water potential $(-1 / \Psi)$ was plotted against the leaf relative water content $(R W C$, derived as the fraction of saturated water present in the leaf at each stage of the dehydration process) to find $\Psi_{\text {TLP. At }}$. water potentials below turgor loss point, the relationship between $-1 / \Psi$ and $R W C$ is linear and $\Psi_{\text {TLP }}$ is determined as the point where 
the relationship becomes non-linear (Due to zero turgor within cells; Tyree and Hammel 1972; Bartlett et al. 2012).

$R W C_{\mathrm{TLP}}$ is the relative water content at $\Psi_{\mathrm{TLP}}$. After $\Psi_{\mathrm{TLP}}$, the continued measurement of leaf water potential is an assessment of the change in solute potential. Thus, a straight line fitted to the points below turgor loss extrapolated to the $y$-axis $(-1 / \Psi)$ is used to find $1 / \pi_{\mathrm{o}}$, the inverse of $\pi_{\mathrm{o}}$. The slope between full leaf hydration and turgor loss point describes stress on the cell wall resulting from dehydration and is equal to $\varepsilon$. $C_{\mathrm{FT}}$ is calculated as $\Delta R W C / \Delta \Psi$ between full turgor and turgor loss point; $C_{\mathrm{TLP}}$ is $\Delta R W C / \Delta \Psi$ between turgor loss point and -2.5 MPa.

Leaves from plants in this study were collected for determination of specific leaf area $(S L A)$. We utilized a greater number of plants from each population for $S L A$ analysis than for determination of pressure volume curves (110 invasive individuals, 729 native individuals for $S L A$ analysis). Leaf surface area was found using a CID Bioscience CI-203 Handheld Laser Leaf Area Meter with a CI-203 CA Conveyer Attachment (CID Bioscience, Camas, WA, USA). Leaves were then gathered and dried in an oven at $60^{\circ} \mathrm{C}$ to constant weight. SLA was calculated as leaf surface area in $\mathrm{m}^{-2} \mathrm{~kg}$ leaf dry weight per individual.

Common Garden Study 2: Morphological Traits

Plants from thirty-three invasive and native populations of $B$. sylvaticum were grown in a common garden experiment from August 2012 to December 2012 ( $\mathrm{n}=5$ individuals per population). Plants were grown from clonal tillers of individuals utilized for pressure-volume curves. Three study plots were established in $1 \mathrm{~m}^{3}$ raised bed plots 
in the research glasshouse at Portland State University, Portland, Oregon, USA. Boxes were filled with sandy loam soil ( $5 \%$ clay, $45 \%$ sand, and 50\% silt). Five tillers, (with the exception of populations ESH, M3, M7, TUK, UK4, which had 4 tillers, and M16, which had 3 tillers) representing different genotypes from each population of $B$. sylvaticum were randomly placed in each raised beds at a fixed distance of $10 \mathrm{~cm}$ from one another.

Two leaf samples were collected from each individual; the most recently fully expanded leaf was utilized for stomatal index $(S I)$ measurements. A second fully expanded leaf was collected and placed in $2.0 \mathrm{~mL}$ vials filled with a 200:5 50\% ethanolglacial acetic acid (17.5M) mixture to preserve tissue for analysis of metaxylem characteristics. Stomatal and metaxylem counts and measurements were made using a Leica MZ16 stereomicroscope (Leica Microsystems, Wetzlar, Germany) linked to a QImaging Retiga 1300 camera (Q-Imaging, Surrey, British Columbia, Canada) and analyzed with Image-Pro 6.0 Software (Media Cybernetics, Warrendale, PA, USA).

Leaf epidermal impressions were obtained according to methods in Beerling and Chaloner (1992) for calculation of $S I$ [stomatal density/(stomatal density + epidermal cell density) x 100, where stomata consist of the stomatal pore and two flanking guard cells], a measurement that normalizes leaf stomatal density in relation to the confounding influence of epidermal cell expansion that may be initiated by factors other than those influencing stomatal development (Royer 2001; Xu and Zhou 2008).

Cross sections of preserved leaf samples were photographed and measured for metaxylem and bulliform cell characteristics on at least 3 individuals per population. Images were analyzed with ImageJ (National Institutes of Health, Bethesda, Maryland, 
USA). Measurements on each section included cross-sectional area, area of the major veins, and proportion of bulliform cells and vascular tissue to cross-sectional area. These measurements were utilized to obtain hydraulically weighted mean diameter $(H M D)$, metaxylem vessel frequency $(V F)$, maximum metaxylem vessel diameter $(M V D)$, and bulliform area $(B A)$ for each individual (Table 4.2). We applied the principle of the Hagen- Poiseuille relationship stating that a conduit's hydraulic conductivity is proportional to its diameter to the $4^{\text {th }}$ power to assess average metaxylem diameter. Specifically, to find $H M D$, we analyzed raw measurements of diameter were analyzed for their frequency in $1 \mu \mathrm{m}$ size classes and for the relative contribution of each class of diameter to the sum of all the conduits raised to the $4^{\text {th }}$ power calculated as:

$H M D=2 \frac{\Sigma \mathrm{r}^{5}}{\Sigma \mathrm{r}^{4}}$, where $\mathrm{r}$ is the radius of a xylem conduit. This equation weights the importance of radii in proportion to the estimated hydraulic conductance of the xylem conduits (Sperry and Saliendra 1994). $V F$ was calculated as the average number of metaxylem vessels per leaf area. $M V D$ is the largest metaxylem vessel per individual. The cross-sectional area of bulliform cells, large leaf surface cells that are the first to lose turgor in times of water stress, resulting in leaves rolling in to prevent transpirational water loss, was found for each individual. $B A$ was calculated as the proportion of bulliform cells present per cross-sectional area (Table 4.2).

Statistical Analysis

The structure of variation in quantitative traits between invasive and native regions of B. sylvaticum was analyzed by running two ANOVA models (un-weighted and weighted by the probability of genetic contribution) for each trait. For weighted models, all invasive populations were given a weight of 1 . The models also included population 
nested within regions and raised bed (raised bed is included for traits measured in Common Garden Study 2 only) treated as random effects. A separate ANOVA was run utilizing only individuals from invasive populations to determine if further differentiation occurred after invasion (Post-invasion differentiation). Response variables were transformed to achieve assumptions of normality for ANOVA (log-transformations: SI, $V F, M V D, H M D, B A, \Psi_{\mathrm{TLP}}, \pi_{\mathrm{o}}, \varepsilon$; square-root transformation: $S L A$; squaredtransformation: $R W C_{\mathrm{TLP}}$; arcsin transformation: $\left.C_{\mathrm{TLP}}, C_{\mathrm{FT}}\right)$. ANOVA analyses were performed in R: Statistical Software (R Core Team 2013). $Q_{S T}-F_{S T}$ Comparisons

To assess whether phenotypic divergence in invasive populations of B. sylvaticum was greater or less than that expected under neutrality, we performed two sets of $Q_{\mathrm{ST}}-F_{\mathrm{ST}}$ comparisons for twelve functional traits $\left(S I, S L A, V F, M V D, H M D, B A, \Psi_{\mathrm{TLP},} \pi_{\mathrm{o}}, \varepsilon, C_{\mathrm{FT}}\right.$, $\left.R W C_{\mathrm{TLP}}, C_{\mathrm{TLP}}\right)$ in $B$. sylvaticum. The first set of comparisons examined pre-invasion differentiation, consisting of an analysis of traits between native and invasive populations. Invasive populations were given a weight of 1 . The second set of comparisons tested post-invasion differentiation, focused solely on invasive Oregon populations, and was not weighted.

For determination of weighted $Q_{\mathrm{ST}}-F_{\mathrm{ST}}$, individuals were grouped into two populations determined by origin, "Native" or "Invasive." Because native populations did not contribute equally to the genetics of Oregon populations, $Q_{\mathrm{ST}}$ and $F_{\mathrm{ST}}$ were weighted by the assignment probabilities calculated from microsatellite data in Geneclass2 (Piry et al. 2004). Weighted values for $F_{\mathrm{ST}}$ were calculated as the weighted mean of pairwise $F_{\mathrm{ST}}$ values between each native population and the grouped "Invasive" population. Pairwise 


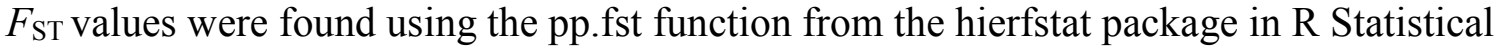
Software (Goudet 2005).

We calculated $Q_{\mathrm{ST}}$ as:

$Q s t=\frac{V_{a}\left(1+F_{I S}\right)}{V_{a}\left(1+F_{I S}\right)+\left(\left(4-\left(2 F_{I S}\right)\right) V_{w}\right.}$

utilizing a form of the equation integrating $F_{\text {IS }}$ to describe the relationship between individuals collected from wild populations. We used $F_{\text {IS }}=0.489$--the value found from grouping invasive populations, calculated in FSTAT (Table 1; Goudet 1995). Weighted values for $V_{\mathrm{a}}$ were determined calculating weighted means for each trait per region and utilizing ANOVA to obtain pairwise variance across the regions. Weighted $V_{\mathrm{w}}$ was calculated as the mean of the variance of each trait per region found by weighted ANOVA. Analysis of post-establishment differentiation was performed similarly using data for comparison among invasive populations, but excluding weights.

Because our experimental procedure measured variance at only eight neutral loci, we utilized a simulation method based on parametric bootstrapping to create a null distribution of $Q_{\mathrm{ST}}-F_{\mathrm{ST}}$ for testing hypotheses of trait evolution in $B$. sylvaticum (Whitlock and Guillaume 2009). Since we cannot measure neutral expectations at each locus, this bootstrapping procedure is necessary to determine if apparent differences between $Q_{\mathrm{ST}}$ and $F_{\mathrm{ST}}$ exceed (or fall below) values expected by chance. Null distributions of $Q_{\mathrm{ST}}$ and $F_{\mathrm{ST}}$ were generated for each trait by bootstrapping the weighted variance components $V_{\mathrm{a}}$ and $V_{\mathrm{w}} 10,000$ times across values. For tests of differentiation during establishment, weights were applied at each iteration prior to resampling to determine the weighted values for the null distribution of $V_{\mathrm{a}}$ and $V_{\mathrm{w}}$. Weighted values of $F_{\mathrm{ST}}$ were 
randomly sampled at each iteration from an estimated distribution created by bootstrapping the microsatellite data across loci and subtracting it from $Q_{\mathrm{ST}}$ to obtain the null distribution of $Q_{\mathrm{ST}}-F_{\mathrm{ST}}$. For post-establishment differentiation tests, weights were excluded. Actual values of $Q_{\mathrm{ST}}-F_{\mathrm{ST}}$ for each trait were compared to the $95 \%$ distribution of the simulated $Q_{\mathrm{ST}}-F_{\mathrm{ST}}$ values to indicate uniform selection (quantiles $0-0.025, Q_{\mathrm{ST}}-F_{\mathrm{ST}}$ $<0$ ) or divergent selection (quantiles $0.975-1, Q_{\mathrm{ST}}-F_{\mathrm{ST}}>0$ ).

\section{Results}

Contribution of Native Populations to the Invasive Region

Results from GeneClass2 found that probabilities of genetic contribution to the invasive population ranged from 0 to 0.17 for native source populations tested (Fig. 4.1). Native populations with the highest contribution to the invasive regions were located in Italy, Greece, Germany, and England.

\section{Trait Variation}

We performed two sets of ANOVAs examining trait variation in twelve functional traits across invasive and native regions of $B$. sylvaticum for tests of preinvasion diversification. For the first set of ANOVAs we utilized data un-weighted by the genetic probability of contribution to invasive regions, and found significant differences in $M V D\left(\mathrm{~F}_{1,73}=4.37, \mathrm{p}=0.04\right), V F\left(\mathrm{~F}_{1,73}=4.06, \mathrm{p}=0.047\right), B A\left(\mathrm{~F}_{1,73}=8.23, \mathrm{p}=0.005\right), \varepsilon$ $\left(\mathrm{F}_{1,138}=5.65, \mathrm{p}=0.019\right)$, and $S L A\left(\mathrm{~F}_{1,837}=18.56, \mathrm{p}<0.0001\right)$. Of these traits, the direction of phenotypic differences between native and invasive regions for $M V D, B A, V F$, and $\varepsilon$ were consistent with hypotheses that invasive $B$. sylvaticum populations have been 
selected for traits improving plant performance in a water-limited environment (Table 4.2, Fig. 4.2).

For the second set of ANOVAs we used data weighted by the genetic probability of contribution to invasive regions, and found that ANOVA models weighted by the genetic probability of contribution yielded different results than un-weighted models. Two functional traits, $B A\left(\mathrm{~F}_{1,60}=5.98, \mathrm{p}=0.017\right)$ and $S L A\left(\mathrm{~F}_{1,636}=14.01, \mathrm{p}=0.0002\right)$ displayed phenotypic differentiation between invasive and native regions. Of these traits, the direction of phenotypic differences between native and invasive regions for $B A$ were consistent with hypotheses of better plant performance in drought stress, while values for SLA were not (Table 4.2, Fig. 4.3).

The third set of ANOVAs examining trait variation within the invasive region (Post-invasion diversification) found no significant variation in trait values within the invasive regions for all functional and fitness traits.

\section{Quantitative genetic divergence}

Unweighted $F_{\mathrm{ST}}$ values for invasive populations only $\left(F_{\mathrm{ST}}=0.461\right)$, was higher than $F_{\mathrm{ST}}$ of the native source populations $\left(F_{\mathrm{ST}}=0.430\right)$, although both values indicate high population structure across regions. Weighted pairwise $F_{\mathrm{ST}}$ values used for $Q_{\mathrm{ST}}-F_{\mathrm{ST}}$ comparisons between native and invasion regions were moderately lower $\left(F_{\mathrm{ST}}=0.3722\right)$, indicating moderate population structure between invasive and contributing native source populations. Overall pre-invasion $Q_{\mathrm{ST}}$ estimates ranged from 0.02 to 0.97 (Table 4.3). For pre-invasion tests of diversification, $Q_{\mathrm{ST}}$ was significantly greater than $F_{\mathrm{ST}}$ for $\Psi_{\mathrm{TLP}}, S I$, $V F, \pi_{\mathrm{o}}, S L A$, and $C_{\mathrm{TLP}}$, indicating adaptive differentiation (Fig. 4.4). Of these traits, SLA was found to be significantly different in the un-weighted ANOVA, but not in the 
direction predicted by hypotheses of adaptation to drought, indicating divergent selection for $S L A$ to a factor other than drought stress. $B A$ displayed significant differences in unweighted ANOVA models but did not have a significant $Q_{\mathrm{ST}}-F_{\mathrm{ST}}$, indicating neutral differentiation.

Although $\Psi_{\mathrm{TLP}}, S I, V F, \pi_{\mathrm{o}}$, and $C_{\mathrm{TLP}}$ were not found to be significantly different between invasive and native regions in ANOVA models, we still consider the findings that $Q_{\mathrm{ST}}>F_{\mathrm{ST}}$ for these traits to be ecologically important; the results demonstrate that populations diverged adaptively despite genetic contributions from native regions. Of these traits, $\Psi_{\mathrm{TLP}}, \pi_{\mathrm{o}}, V F$, and $C_{\mathrm{TLP}}$ display phenotypic differences between native and invasive regions in the direction og predictions of adaptation to drought stress in invasive populations (Fig. 4.3, Fig.4.4).

Post-invasion estimates utilizing only invasive populations displayed $Q_{\mathrm{ST}}$ values ranging from 0.07 to 0.47 (Table 4.4). For post-invasion tests of diversification, no significant $Q_{\mathrm{ST}}-F_{\mathrm{ST}}$ values were found, indicating that no further divergence has occurred in populations after invasive range expansion.

\section{Discussion}

The results from this study indicate that phenotypic divergence exceeding that expected under neutrality has occurred in plants from invasive populations of $B$. sylvaticum. This phenotypic divergence was found between invasive and native populations; however, it was not found across invasive populations. Importantly, this pattern indicates that divergence for traits measured in this study occurred during the 
establishment period of B. sylvaticum prior to invasive range expansion. Our results are consistent with the hypothesis that adaptive evolution during the lag phase (following introduction) may facilitate range expansion and the ultimate success of an invasive species in a novel environment.

One of the main goals of this study was to develop a more accurate method for determination of adaptive genetic differentiation in invasive species. Many previous studies have determined the presence of differentiation or variation in potentially adaptive traits without considering variation in genetic contribution from native-range populations to the newly introduced populations (e.g., Bossdorf et al. 2005; Lavergne and Molofsky 2007; Leishman et al. 2007). The accuracy of the $Q_{\mathrm{ST}}-F_{\mathrm{ST}}$ approach, shown to be reliable for detection of local adaptation and differentiation, can be improved for the purposes of detecting adaptation during invasion when weights describing the probability of genetic contribution of native populations to the invasion are included. In our analysis, the traits displaying significant phenotypic differentiation differed when ANOVA was performed with and without such weighting. Traits facilitating invasion success can vary by taxa and habitat (Hayes and Barry 2008) and more accurate inferences of the evolutionary processes responsible for trait divergence during invasion can be obtained through the weighted $Q_{\text {ST- }} F_{\text {ST }}$ method applied in this study.

Both divergent selection (for $\Psi_{\mathrm{TLP}}, S I, V F, \pi_{\mathrm{o}}, S L A$, and $C_{\mathrm{TLP}}$ ) and neutral differentiation (for $B A$ ) contributed to phenotypic differentiation of native and invasive $B$. sylvaticum populations. Many species become invasive as a result of multiple introductions that increase available genetic diversity, allowing for selection to occur on 
recombinant hybrids not found in a species' native range during the introductory lag phase (Darling et al. 2008; Dlugosch and Parker 2008).

A common hypothesis is that the post-introduction lag phase is a period of slow growth that allows selection to act on introduced populations during which certain adaptations must evolve for continued demographic growth (Mooney and Cleland 2001). However, there are few documented examples of this process in wild populations (Espeland 2013; van Klinken et al. 2015) primarily due to the difficulty of detecting lag phases, which occur prior to invasion in alien species that often go unnoticed. This study shows phenotypic diversification between native and invasive regions occurred during the lag period following introduction rather than during subsequent radiation of populations within the invasive range.

Although invasive B. sylvaticum populations are the result of multiple introductions (Rosenthal et al. 2008), a comparison of $F_{\text {ST }}$ values across the range of native populations $\left(F_{\mathrm{ST}}=0.430\right)$ and within invasive populations alone $\left(F_{\mathrm{ST}}=0.461\right)$ reveal that population differentiation is greater in the invasive region than in the native region. This is expected as founder effects in the invasive range can reduce heterozygosity and increase genetic differentiation across populations (Brown and Marshall 1980). This may be the reason why trait divergence was not found in populations across the invasive region, as bottlenecks resulting from founder effects during invasive spread from the primary site of introduction can reduce genetic diversity and increase genetic drift, reducing phenotypic trait divergence and the probability of local adaptation (Sakai et al. 2001). However, invasive species may also phenotypically diverge after the lag period of invasion. A recent study of three plant species invasive to 
the United Kingdom found that all three species were changing in at least one trait more than a century after their introduction (Flores-Moreno et al. 2015). It is possible that because invasive $B$. sylvaticum populations are the product of hybridization of populations from throughout the native range that portions of parental pre-hybridization genomes have been retained, allowing invasive populations to possess a wide ecological breadth (Rieseberg et al. 1993; Rosenthal et al. 2008).

There was disparity in how invasive $B$. sylvaticum phenotypic differences between native and invasive regions compared to hypotheses of adaptation to drought stress. Differences in $\Psi_{\mathrm{TLP}}, \pi_{\mathrm{o}}, V F$, and $C_{\mathrm{TLP}}$ between invasive and native regions all fit assumptions of adaptation to drought in the invasive region, while $S L A$ and $S I$ did not. This pattern may indicate that environmental factors other than water limitation have played a role in diversification of the latter traits. While drought is a significant environmental stress, other climatic factors could result in opposing changes in functional traits. For example, $S L A$ is also influenced by nitrogen gradients in soil, where larger amounts of available nitrogen can result in greater SLA (Knops and Reinhart 2000), while increased light availability can reduce SI (Schoch et al. 1980). Results contrary to predictions of adaptation to drought stress may also be the result of functional trade-offs between traits. These trade-offs are plant water-use strategies to increase resource capture and retention rates and can change with environment (Grime 2001).

Though phenotypic shifts towards more adaptive trait values in the invasive environment can increase the chances of invasion success, previous work has shown that other factors can lead to the proliferation of B. sylvaticum in its invasive habitat. Roy et al. (2011) examined the effect of enemy release on the invasive potential of $B$. 
sylvaticum. In native regions, plants can become infected with an endophytic fungus preventing sexual reproduction, while the infectious endophyte does not occur in invasive regions. It was found that the fungus inhibited seedling survival in the native range (Roy et al. 2011). Additionally, B. sylvaticum invasion is associated with sites that have a recent history of physical disturbances such as logging that aid primary colonization (Taylor and Cruzan 2015). Moreover, several mechanisms have been found to reduce the effects of bottlenecks in the invasive region for B. sylvaticum. Increases in nitrogen deposition have been found the increase fitness of $B$. sylvaticum individuals from genetically depauperate populations (Marchini and Cruzan unpublished). Marchini et al. (unpublished) found evidence that genetic purging, selection against deleterious alleles expressed in the homozygous state of an inbreeding population, was occurring in invasive populations. Genetic purging and nitrogen deposition can increase plant fitness despite a low effective population size, instigating additional invasion into new areas (Chapter 2 and Chapter 3). The successful invasion of B. sylvaticum into the Pacific Northwest USA appears to be product of enemy release, site disturbance, nitrogen deposition, and phenotypic shifts towards traits more suited towards climate of the invasive range. Rapid adaptation is considered to be an important process underlying species invasion in an introduced range. We found that the phenotypic shifts in our measured traits likely occurred during the pre-invasive, establishment phase of B. sylvaticum's introduction. By identifying the phase of invasion where adaptation to the new environment is most likely to occur, management strategies can be directed towards populations with the most potential to spread. We have also utilized information on the putative source regions of B. sylvaticum to determine a more accurate method for 
detection of trait differentiation in the invasive range. Application of this robust method for detection of phenotypic differentiation in invasive populations allows for more accurate detection of traits leading to invasiveness in non-native species.

Acknowledgements We thank C. Maraist, B. Meyer, T. Musial, T. Rosenstiel, and M. Williamson for technical assistance, S. Jaconis and B. Kohrn for comments on the manuscript, and M. Poyourow, P. Catalan, and M. Ristow for providing B. sylvaticum seeds for this study. 


\section{Tables and Figures}

Table 4.1 Location, latitude and longitude, sample size (n), gene diversity $\left(H_{\mathrm{S}}\right)$, and inbreeding coefficients $\left(F_{\text {IS }}\right)$ for populations sampled in this study.

\begin{tabular}{|c|c|c|c|c|c|c|}
\hline Code & Location & Latitude & Longitude & $\mathrm{N}$ & $H s$ & Fis \\
\hline & NATIVE & & & & & \\
\hline$\overline{\mathrm{ESH}}$ & England, Shropshire & $52^{\circ} 37^{\prime} 32^{\prime \prime}$ & $-3^{\circ} 44^{\prime} 14^{\prime \prime}$ & 8 & 0.352 & 0.422 \\
\hline ES & England, Surrey & $51^{\circ} 23^{\prime} 24^{\prime \prime}$ & $-1^{\circ} 22^{\prime} 48^{\prime \prime}$ & 8 & 0.08 & 0.167 \\
\hline SPIN & Huesca, Spain & $42^{\circ} 8^{\prime} 12^{\prime \prime}$ & $-1^{\circ} 35^{\prime} 24^{\prime \prime}$ & 8 & 0.299 & 0.843 \\
\hline TUN & Tunisia & $34^{\circ} 32^{\prime} 29^{\prime \prime}$ & $9^{\circ} 13^{\prime} 20^{\prime \prime}$ & 8 & 0.253 & 0.383 \\
\hline Gerh & German Halle & $51^{\circ} 28^{\prime} 12^{\prime \prime}$ & $11^{\circ} 57^{\prime} 36^{\prime \prime}$ & 6 & 0.55 & 0.742 \\
\hline Gerl & German Leipzig & $51^{\circ} 20^{\prime} 24^{\prime \prime}$ & $12^{\circ} 22^{\prime} 48^{\prime \prime}$ & 6 & 0.433 & 0.712 \\
\hline IC & Italy, Calabria & $39^{\circ} 31^{\prime} 48^{\prime \prime}$ & $16^{\circ} 12^{\prime} 36^{\prime \prime}$ & 8 & 0.56 & 0.442 \\
\hline ICB & Italy, Calabria 2 & $39^{\circ} 32^{\prime} 18^{\prime \prime}$ & $16^{\circ} 12^{\prime} 31^{\prime \prime}$ & 8 & 0.573 & 0.372 \\
\hline IP & Italy, Puglia & $40^{\circ} 52^{\prime} 48^{\prime \prime}$ & $16^{\circ} 45^{\prime} 36^{\prime \prime}$ & 8 & 0.51 & 0.387 \\
\hline GIK & Greece, Kerkira & $39^{\circ} 31^{\prime} 48^{\prime \prime}$ & $19^{\circ} 55^{\prime} 48^{\prime \prime}$ & 8 & 0.516 & 0.273 \\
\hline SES & Slovakia, East Slovakia & $48^{\circ} 49^{\prime} 41^{\prime \prime}$ & $20^{\circ} 9^{\prime} 4^{\prime \prime}$ & 2 & 0.344 & 0.273 \\
\hline SER & Vlakca, Serbia & $44^{\circ} 10^{\prime} 17^{\prime \prime}$ & $20^{\circ} 41^{\prime} 54^{\prime \prime}$ & 8 & 0.063 & 0.5 \\
\hline GII & Greece, Ioannina & $29^{\circ} 40^{\prime} 12^{\prime \prime}$ & $20^{\circ} 50^{\prime} 24^{\prime \prime}$ & 8 & 0.544 & 0.598 \\
\hline GIP & Greece, Ioannina 2 & $29^{\circ} 40^{\prime} 12^{\prime \prime}$ & $20^{\circ} 50^{\prime} 24^{\prime \prime}$ & 8 & 0.493 & 0.208 \\
\hline GTC & Greece, Larrisa & $39^{\circ} 32^{\prime} 47^{\prime \prime}$ & $22^{\circ} 8^{\prime} 11^{\prime \prime}$ & 8 & 0.616 & 0.315 \\
\hline GMT & Greece, Thessaloniki & $40^{\circ} 37^{\prime} 48^{\prime \prime}$ & $22^{\circ} 57^{\prime} 36^{\prime \prime}$ & 8 & 0.338 & 0.492 \\
\hline UKR & Ukrain, Krym & $44^{\circ} 24^{\prime} 14^{\prime \prime}$ & $33^{\circ} 49^{\prime} 30^{\prime \prime}$ & 10 & 0.166 & 0.121 \\
\hline TUR & Turkey, Gerze, Sinop & $41^{\circ} 49^{\prime} 2^{\prime \prime}$ & $35^{\circ} 1^{\prime} 24^{\prime \prime}$ & 8 & 0.662 & 0.646 \\
\hline RUS & Russian Federation & $45^{\circ} 0^{\prime} 24^{\prime \prime}$ & $41^{\circ} 58^{\prime} 29^{\prime \prime}$ & 10 & 0.45 & 0.278 \\
\hline IRAN & Iran & $35^{\circ} 5^{\prime} 21^{\prime \prime}$ & $52^{\circ} 20^{\prime} 20^{\prime \prime}$ & 7 & 0.244 & 0.268 \\
\hline \multirow[t]{2}{*}{ KAZ } & Kazakastan, Alma Ata & $43^{\circ} 16^{\prime} 24^{\prime \prime}$ & $76^{\circ} 55^{\prime} 54^{\prime \prime}$ & 9 & 0.174 & 0.52 \\
\hline & INVAS IVE & & & & & \\
\hline $\mathrm{C} 1$ & Corvallis & $44^{\circ} 39^{\prime} 35^{\prime \prime}$ & $-124^{\circ} 45^{\prime} 41^{\prime \prime}$ & 24 & 0.26 & 0.936 \\
\hline C6 & Hwy 22 & $44^{\circ} 45^{\prime} 19^{\prime \prime}$ & $-123^{\circ} 36^{\prime} 42^{\prime \prime}$ & 24 & 0.133 & 0.438 \\
\hline $\mathrm{C} 10$ & Corvallis & $44^{\circ} 23^{\prime} 29^{\prime \prime}$ & $-124^{\circ} 38^{\prime} 4^{\prime \prime}$ & 24 & 0.133 & 0.645 \\
\hline E1 & Eugene & $43^{\circ} 57^{\prime} 35^{\prime \prime}$ & $-123^{\circ} 15^{\prime} 49^{\prime \prime}$ & 34 & 0.085 & 0.692 \\
\hline E4 & Eugene & $44^{\circ} 0^{\prime} 48^{\prime \prime}$ & $-123^{\circ} 7^{\prime} 30^{\prime \prime}$ & 14 & 0.042 & 0.148 \\
\hline E6 & Eugene & $43^{\circ} 59^{\prime} 47^{\prime \prime}$ & $-123^{\circ} 12^{\prime} 3^{\prime \prime}$ & 24 & 0.37 & 0.817 \\
\hline E7 & Eugene & $43^{\circ} 58^{\prime} 29^{\prime \prime}$ & $-123^{\circ} 21^{\prime} 13^{\prime \prime}$ & 21 & 0.097 & 0.561 \\
\hline E9 & Eugene & $43^{\circ} 58^{\prime} 26^{\prime \prime}$ & $-123^{\circ} 7^{\prime} 36^{\prime \prime}$ & 24 & 0.392 & 0.544 \\
\hline M1 & Fish_Ck_Cmpgrnd & $44^{\circ} 23^{\prime} 54^{\prime \prime}$ & $-123^{\circ} 39^{\prime} 18^{\prime \prime}$ & 15 & 0.033 & 0.429 \\
\hline M16 & Cedar \& Wiley & $44^{\circ} 22^{\prime} 19^{\prime \prime}$ & $-123^{\circ} 22^{\prime} 45^{\prime \prime}$ & 24 & 0.297 & 0.361 \\
\hline S1 & Fisherman's Bend & $44^{\circ} 45^{\prime} 14^{\prime \prime}$ & $-123^{\circ} 28^{\prime} 56^{\prime \prime}$ & 29 & 0.063 & 0.379 \\
\hline
\end{tabular}


Table 4.2 Functional traits measured in B. sylvaticum and their units, description, and expected behavior in drought.

\begin{tabular}{|c|c|c|c|c|c|}
\hline Symbol & Variable & Unit & Significance & Expectations in Drought & References \\
\hline SLA & Specific Leaf Area & $\mathrm{m}^{-2} \mathrm{~kg}$ & Leaf surface area:mass ratio & $\begin{array}{c}\text { Low SLA delays tissue } \\
\text { dehydration. }\end{array}$ & $\begin{array}{l}\text { Larcher (1995), } \\
\text { Poorter et al. (2009) }\end{array}$ \\
\hline SI & Stomatal Index & $\%$ & $\begin{array}{l}\text { Normalized measurement of } \\
\text { stomatal density }\end{array}$ & $\begin{array}{c}\text { Many small stomata can } \\
\text { increase water-use efficiency. }\end{array}$ & $\begin{array}{c}\text { Beerling \& Chaloner } \\
\text { (1992), Xu \& Zhou } \\
\text { (2008) }\end{array}$ \\
\hline VF & Vessel Frequency & $\%$ & $\begin{array}{c}\text { Xylem vessel frequency per } \\
\text { leaf area. }\end{array}$ & $\begin{array}{l}\text { Xylem vessel size decreases } \\
\text { while vessel frequency } \\
\text { increases }\end{array}$ & $\begin{array}{l}\text { Tyree \& Zimmerman } \\
\text { (2002), Smith et al. } \\
\text { (2013) }\end{array}$ \\
\hline$M V D$ & $\begin{array}{l}\text { Maximum Vessel } \\
\text { Diameter }\end{array}$ & $\mu \mathrm{m}$ & $\begin{array}{c}\text { Diameter of the largest xylem } \\
\text { vessel per individual }\end{array}$ & $\begin{array}{l}\text { Plants in dry environments } \\
\text { have smaller xylem vessels. }\end{array}$ & $\begin{array}{c}\text { Tyree \& Zimmerman } \\
\text { (2002) }\end{array}$ \\
\hline$H M D$ & $\begin{array}{l}\text { Hydraulically } \\
\text { Weighted Mean } \\
\text { Vessel Diameter }\end{array}$ & $\mu \mathrm{m}$ & $\begin{array}{c}\text { Xylem diameter; accounting for } \\
\text { hydraulic resistance. }\end{array}$ & $\begin{array}{l}\text { Less resistance from xylem } \\
\text { conduits increases water flow. }\end{array}$ & $\begin{array}{l}\text { Tyree \& Zimmerman } \\
\text { (2002), Tombesi et } \\
\text { al. (2010) }\end{array}$ \\
\hline$B A$ & Bulliform Cell Area & $\mu \mathrm{m}$ & $\begin{array}{c}\text { Water loss results in bulliform } \\
\text { cells shinking to reduce leaf } \\
\text { surface area }\end{array}$ & $\begin{array}{l}\text { Smaller bulliform cells result in } \\
\text { rapid leaf rolling. }\end{array}$ & $\begin{array}{l}\text { Kadioglu et al. } \\
\qquad \text { (2012) }\end{array}$ \\
\hline$\Psi_{\mathrm{TLP}}$ & $\begin{array}{l}\text { Water potential at } \\
\text { turgor loss point }\end{array}$ & $\mathrm{MPa}$ & $\begin{array}{l}\text { The point at which leaf cells } \\
\text { lose turgor, leaf wilting point. }\end{array}$ & $\begin{array}{l}\text { More negative } \Psi_{\text {TLP }} \text { indicates } \\
\text { greater turgor maintenance. }\end{array}$ & $\begin{array}{l}\text { Meinzer \& Grantz } \\
\text { (1990), Aroca et al. } \\
\text { (2012) }\end{array}$ \\
\hline$R W C_{\mathrm{TLP}}$ & $\begin{array}{c}\text { Relative Water } \\
\text { Content at Turgor Loss } \\
\text { Point }\end{array}$ & $\%$ & $\begin{array}{l}\text { Cell hydration at turgor loss } \\
\text { point }\end{array}$ & $\begin{array}{l}\text { Higher } R W C_{\text {TLP }} \text { delays leaf } \\
\text { dessication after stomatal } \\
\text { closure, reduces cell wall } \\
\text { damage. }\end{array}$ & $\begin{array}{c}\text { Tyree \& Zimmerman } \\
\text { (2002), Bartlett et } \\
\text { al. }(2012)\end{array}$ \\
\hline$\varepsilon$ & $\begin{array}{c}\text { Modulus of Elasticity } \\
\text { at Full Turgor }\end{array}$ & $\mathrm{MPa}$ & $\begin{array}{c}\text { Deformability of a cell wall; } \\
\text { pressure change required to } \\
\text { cause a unit change in cell } \\
\text { volume. }\end{array}$ & $\begin{array}{l}\text { Rigid walls (high } \varepsilon \text { ) slows cell } \\
\text { volume declines during } \\
\text { drought. }\end{array}$ & $\begin{array}{c}\text { Verslues \& Zhu } \\
\text { (2005), Aroca et al. } \\
\text { (2012) }\end{array}$ \\
\hline$C_{\mathrm{TLP}}$ & $\begin{array}{c}\text { Capacitance at Turgor } \\
\text { Loss Point }\end{array}$ & $\mathrm{MPa}$ & $\begin{array}{l}\text { Water storage capacity of } \\
\text { plant at turgor loss point. }\end{array}$ & $\begin{array}{c}\text { High capacitance maintains } \\
\text { cell hydration, reductes cell } \\
\text { wall damage. }\end{array}$ & Bartlett et al. (2012) \\
\hline$C_{\mathrm{FT}}$ & $\begin{array}{c}\text { Capacitance at Full } \\
\text { Turgor }\end{array}$ & $\mathrm{MPa}$ & $\begin{array}{c}\text { Water storage capacity of } \\
\text { plant at full turgor. }\end{array}$ & $\begin{array}{l}\text { High capacitance enables leaf } \\
\text { cells to store more water, } \\
\text { increasing water potentials; } \\
\text { buffer against water }\end{array}$ & $\begin{array}{l}\text { Lamont \& Lamont } \\
\text { (2000), Sack et al. } \\
\text { (2005) }\end{array}$ \\
\hline$\pi_{\mathrm{o}}$ & $\begin{array}{l}\text { Osmotic Potential at } \\
\text { Full Turgor }\end{array}$ & $\mathrm{MPa}$ & $\begin{array}{c}\text { Solute concentration in plant } \\
\text { cells. }\end{array}$ & $\begin{array}{l}\text { More negative osmotic } \\
\text { potential allows maintenance } \\
\text { of leaf turgor at lower water }\end{array}$ & $\begin{array}{c}\text { Verslues \& Zhu } \\
\text { (2005), Aroca et al. } \\
\text { (2012) }\end{array}$ \\
\hline
\end{tabular}


Table 4.3 Variance components, $F_{\mathrm{ST}}, Q_{\mathrm{ST}}$, and $Q_{\mathrm{ST}}-F_{\mathrm{ST}}$ for populations across the native and invasive ranges of $B$. sylvaticum, using $F_{\mathrm{ST}}=0.3722$.

\begin{tabular}{lllll} 
Trait & $V_{\mathrm{a}}$ & $V_{\mathrm{w}}$ & $Q_{\mathrm{ST}}$ & $Q_{\mathrm{ST}-F_{\mathrm{ST}}}$ \\
\hline$\pi_{\mathrm{o}}$ & 1.009098 & 0.4378309 & 0.5317487 & $\mathbf{0 . 1 5 9 4 9 *}$ \\
$\Psi_{\mathrm{TLP}}$ & 2.054802 & 0.5899417 & 0.631835 & $\mathbf{0 . 2 5 9 5 8 1 3}$ \\
$R W C_{\mathrm{TLP}}$ & 47.2735 & 100.1227 & 0.1887335 & -0.1835202 \\
$\varepsilon$ & 100.7726 & 961.5946 & 0.04910042 & -0.32315328 \\
$C_{\mathrm{FT}}$ & 0.000163263 & 0.003584595 & 0.02194875 & -0.35030495 \\
$C_{\mathrm{TLP}}$ & 0.01064539 & 0.006101257 & 0.4622764 & $\mathbf{0 . 0 9 0 0 2 2 7 ^ { * }}$ \\
$V F$ & 156.7148 & 3.851431 & 0.9524913 & $\mathbf{0 . 5 8 0 2 3 7 6}$ \\
$M V D$ & 0.00151247 & 0.001567612 & 0.3222124 & -0.05004133 \\
$H M D$ & 0.0056935 & 0.004712658 & 0.3731467 & 0.000892966 \\
$S I$ & 304.1888 & 5.165326 & 0.966685 & $\mathbf{0 . 5 9 4 4 3 1 3}$ \\
$S L A$ & 24.24337 & 0.599991 & 0.9099225 & $\mathbf{0 . 5 3 7 6 6 8 8}$ \\
$B A$ & $3.39 \mathrm{E}-06$ & $5.03 \mathrm{E}-07$ & 0.7687541 & 0.3965004 \\
\hline
\end{tabular}

*Indicates significance at $\mathrm{p}<0.05$ 
Table 4.4 Variance components, $F_{\mathrm{ST}}, Q_{\mathrm{ST}}$, and $Q_{\mathrm{ST}}-F_{\mathrm{ST}}$ for populations in the invasive range of $B$. sylvaticum, using $F_{\mathrm{ST}}=0.4609$.

\begin{tabular}{lllll} 
Trait & $V_{\mathrm{a}}$ & $V_{\mathrm{w}}$ & $Q_{\mathrm{ST}}$ & $Q_{\mathrm{ST}}-F_{\mathrm{ST}}$ \\
\hline$\pi_{\mathrm{o}}$ & 7.801051 & 31.0175 & 0.1102581 & -0.3506419 \\
$\Psi_{\mathrm{TLP}}$ & $5.92 \mathrm{E}+00$ & $2.60 \mathrm{E}+01$ & 0.1008121 & -0.3600879 \\
$R W C_{\mathrm{TLP}}$ & 36.58257 & 146.8322 & 0.1093369 & -0.3515631 \\
$\varepsilon$ & 9102.645 & 33672.61 & 0.1175401 & -0.3433599 \\
$C_{\mathrm{FT}}$ & $1.83 \mathrm{E}-05$ & $4.60 \mathrm{E}-06$ & 0.1098412 & -0.3510588 \\
$C_{\mathrm{TLP}}$ & $5.12 \mathrm{E}-06$ & $2.31 \mathrm{E}-05$ & 0.09839043 & -0.3625096 \\
$V F$ & 2.064549 & 7.140013 & 0.1247043 & -0.3361957 \\
$M V D$ & 0.000808006 & 0.002710409 & 0.1280737 & -0.3328263 \\
$H M D$ & 0.002291911 & 0.007696083 & 0.1279575 & -0.3329425 \\
$S I$ & 3.853779 & 8.074664 & 0.190388 & -0.270512 \\
$S L A$ & 21.23948 & 83.0079 & 0.1119587 & -0.3489413 \\
$B A$ & $2.29 \mathrm{E}-07$ & $8.98 \mathrm{E}-07$ & 0.1115797 & -0.3493203 \\
\hline
\end{tabular}

*Indicates significance at $\mathrm{p}<0.05$ 
Figure 4.1 Probability of genetic contribution to invasive populations of $B$. sylvaticum from 21 populations from throughout the native range.

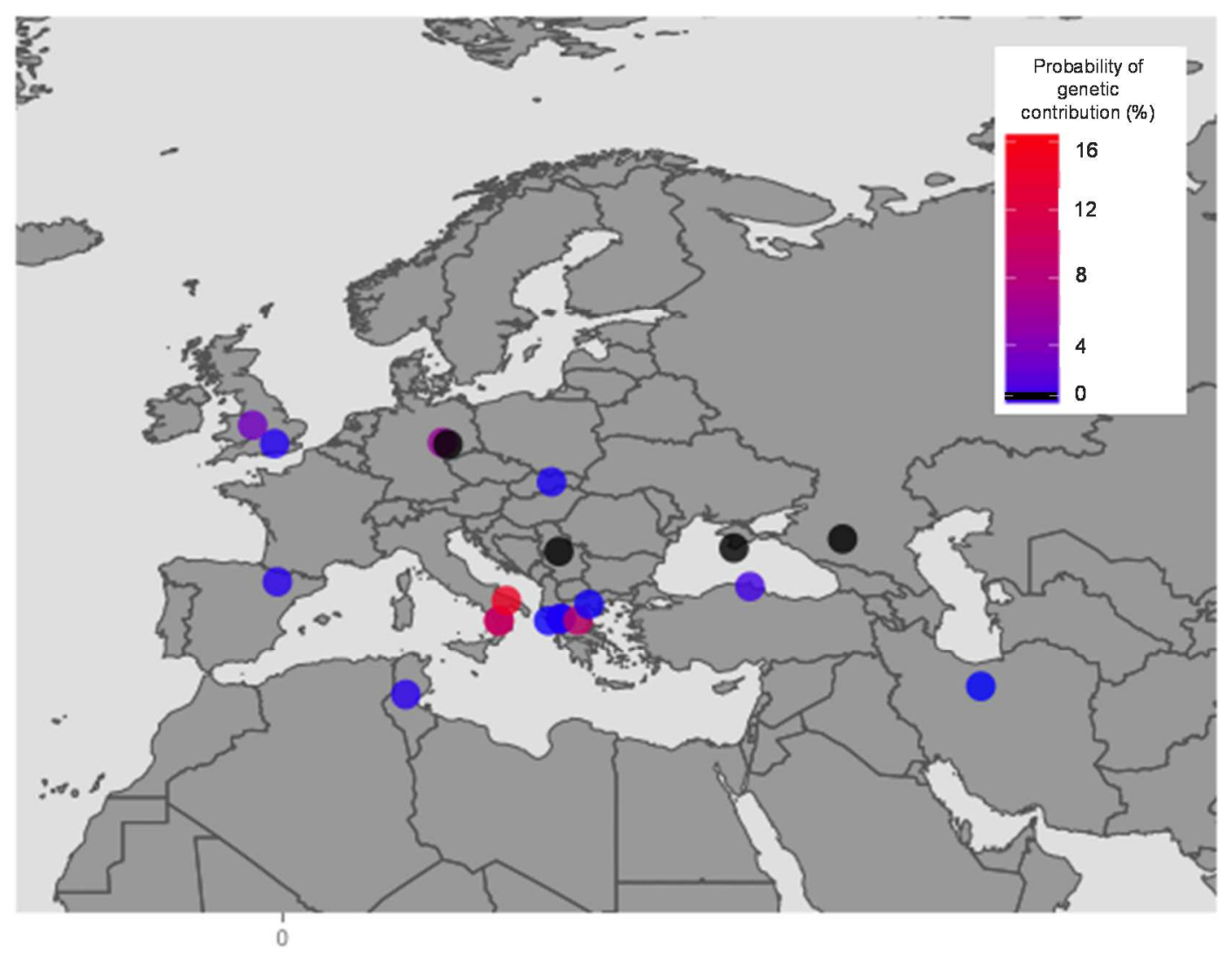


Figure 4.2 Traits exhibiting significant differences at $p<0.05$ between invasive and native population using data unweighted by the probability of genetic contribution.
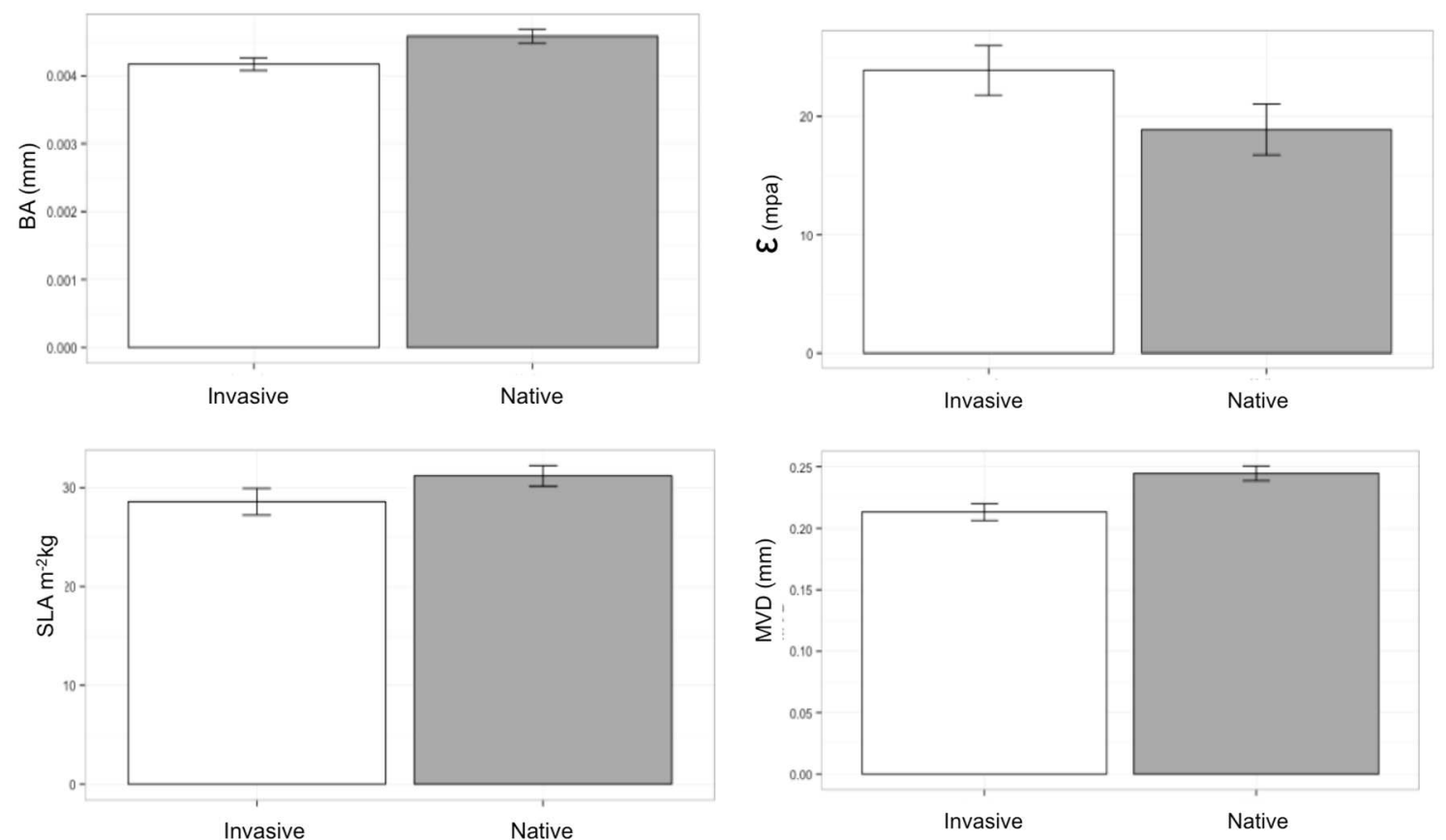
Figure 4.3 Traits exhibiting significant differences at $p<0.05$ between invasive and native population using data weighted by the probability of genetic contribution.
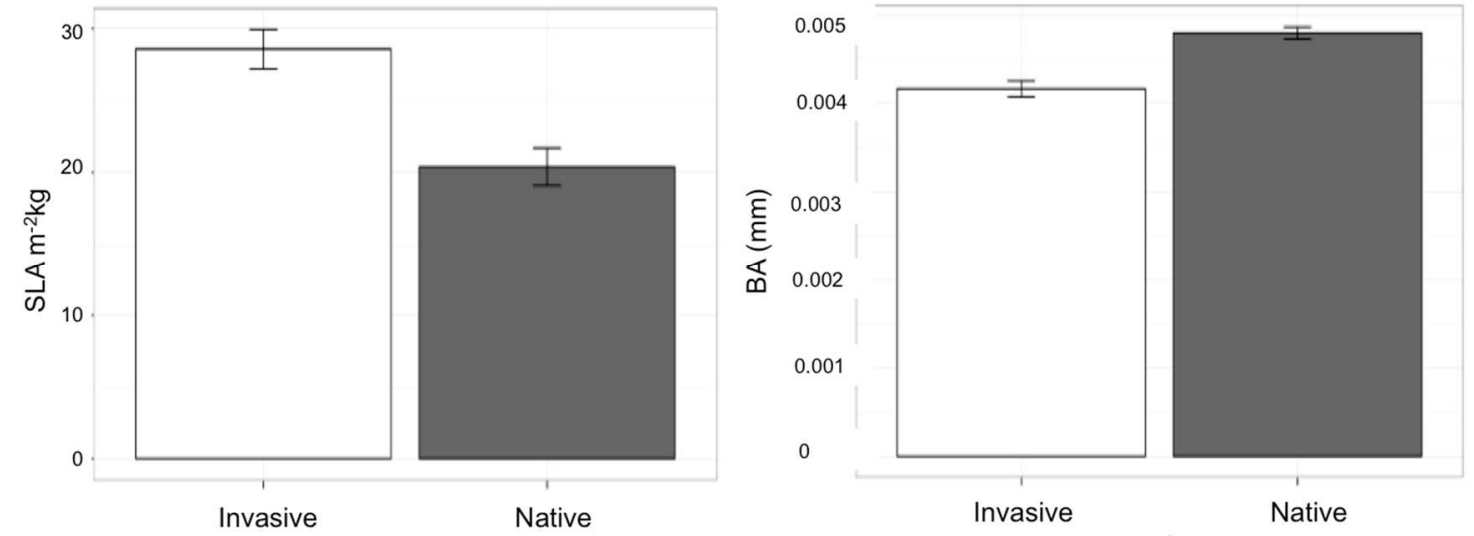
Figure 4.4 Null distributions of $Q_{\mathrm{ST}}-F_{\mathrm{ST}}$ for five quantitative traits with significant values of $Q_{\mathrm{ST}}-F_{\mathrm{ST}}$ in $B$. sylvaticum, indicating selective divergence in the invasive range. Black arrows indicate actual values of $Q_{\mathrm{ST}}-F_{\mathrm{ST}}$ for traits.
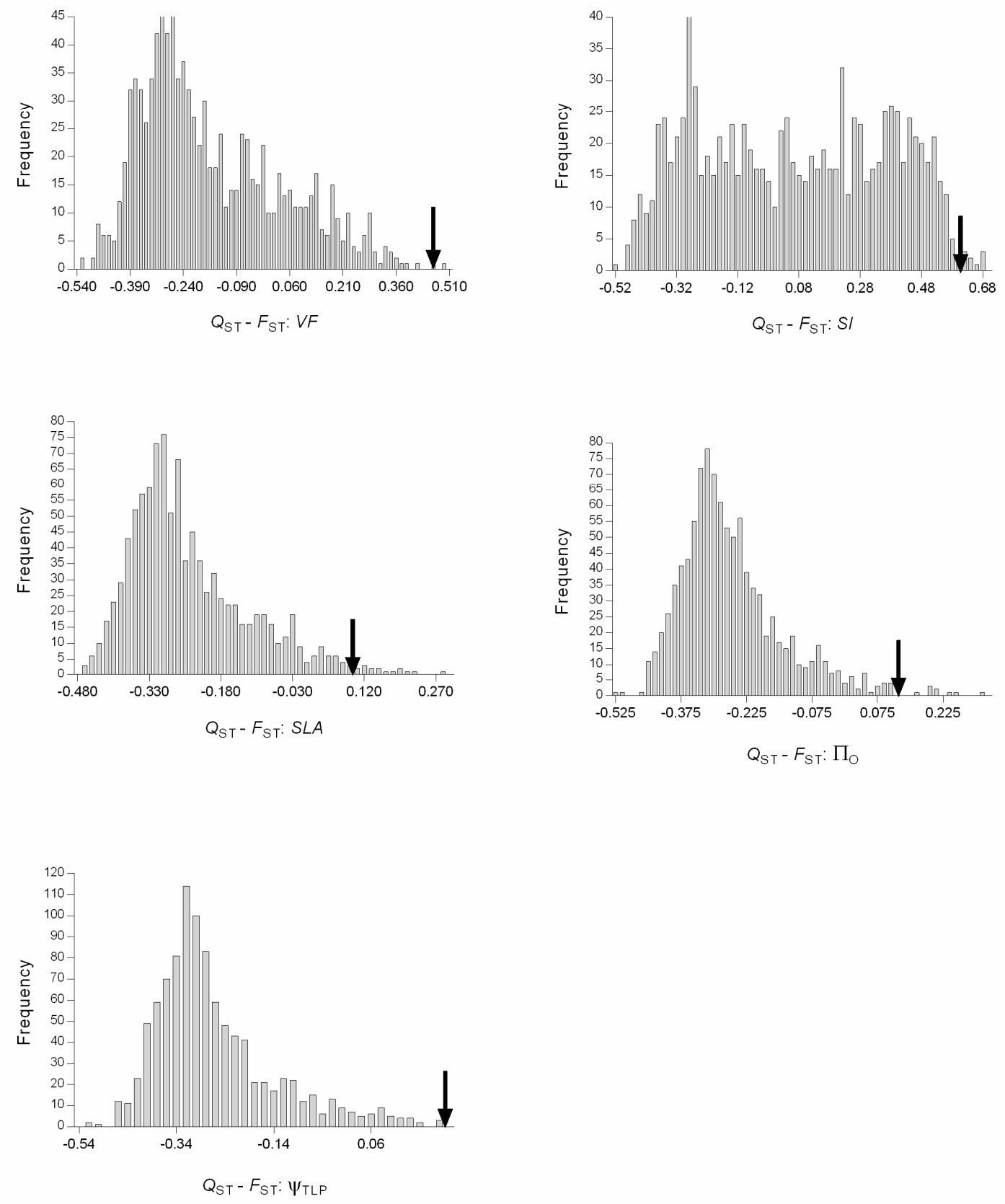


\section{Chapter 5}

Trait divergence, not plasticity, determines the success of a newly invasive plant

\section{Abstract:}

- Phenotypic plasticity and genetic differentiation play important roles in the establishment and spread of invasive species; however, previous studies investigating the role of plasticity in invasion have focused on morphological traits associated with fitness. Here, we determine the adaptive value of phenotypic plasticity and genetic divergence for functional traits related to water uptake in an invasive plant.

- We measured six morphological traits in contrasting water environments for individuals from invasive and native ranges of the bunchgrass Brachypodium sylvaticum (Hudson) Beauv. To accurately represent genotypes contributing to invasion, we used the presence of unique alleles to calculate probabilities genetic contribution from native populations, and used these probabilities as weights in our analyses. Regression-based linear and nonlinear selection coefficients were estimated to determine the adaptive value of plasticity and genetic differentiation.

- Plasticity was absent in response to water availability. Plants in the invasive range had increased xylem vessel frequency, smaller xylem diameter, and lower bulliform cell area than plants from the native range, indicating a shift in the invasive range towards drought tolerant phenotypes. Stomatal density was associated with climate of the invasive range, implying local adaptation to waterlimitation. Genetic divergence of invasive plants was not consistently in the 
direction indicated by selection coefficients, suggesting limitations of selection that may be a consequence of physical constraints and/or tradeoffs between growth and abiotic tolerance.

- Differentiation of functional traits is the consequence of post-introduction selection in the invasive range; although the adaptive value of traits is environmentally determined. Plasticity of functional traits appears to be unimportant and in some cases may impose a liability on invasion success. 


\section{INTRODUCTION}

Phenotypic plasticity and evolutionary change are thought to play major roles in the establishment and spread of invasive species (Lambrinos 2004; Dybdahl and Kane 2005; Geng et al. 2007; Dlugosch and Parker 2008). Upon primary introduction to a novel habitat plasticity may provide plants with the ability to occupy a wide range of environments, promoting invasive establishment and spread (Droste et al. 2010; Davidson et al. 2011; Martina and von Ende 2012). Morphological differences have been found across the invasive and native range of plant species (Alexander et al. 2009;

Eriksen et al. 2012; Felker - Quinn et al. 2013), however the adaptive value of these trait shifts in the invasive range have not been investigated. Additionally, the morphological traits studied tend to be associated with fitness (reviewed in: Davidson et al. 2011; Godoy et al. 2011; Palacio - López and Gianoli 2011). Here, we investigate the role of adaptation in plasticity and genetic differentiation of functional traits for the success of an invasive plant.

Phenotypic plasticity is defined as the ability of organisms to modify morphological or physiological traits in response to environmental conditions (Turesson 1922), and can be adaptive if plasticity increases the ability of a species to survive and reproduce across environments in comparison to non-plastic genotypes (Callaway et al. 2003; Pigliucci and Murren 2003; Richards et al. 2006; Ghalambor et al. 2007). The ability of invasive plants to colonize a novel environment is commonly attributed to species possessing generalist genotypes with high phenotypic plasticity, as plasticity allows introduced individuals to shift traits towards optimal values favored by the local environment (Matesanz et al. 2012; Zhao et al. 2012). For example, plants that exhibit 
plasticity in response to drought stress tolerate limited water availability by shifting morphological or physiological trait expression to meet environmental optima (Picotte et al. 2007; Meier and Leuschner 2008; Bartlett et al. 2012). There is evidence that phenotypic plasticity is common in invasive species in response to nutrient, light, and water availability (Pattison et al. 1998; Niinemets et al. 2003; Burns and Winn 2006; Davidson et al. 2011). Adaptive plasticity has been found in invasive plant populations in response to climate (Hahn et al. 2012; Zenni et al. 2014; Bock et al. 2015; Turner et al. 2015), but detailed evaluations of the contribution of plasticity for functional traits to invasion success are lacking.

Selection in the invasive range is thought to allow species to adapt to novel environments (Willis et al. 2000b; Muller-Scharer et al. 2004; Keller and Taylor 2008; Prentis et al. 2008; Whitney and Gabler 2008). Selective pressures can lead to genetic differentiation, displayed as shifts in trait values between individuals from invasive and native ranges that are not a result of plasticity. Post-introduction selection can lead to local adaptation to the new abiotic environment, resulting in genetic differentiation that supports population persistence (Piersma and Drent 2003; Prentis et al. 2008). Evidence of genetic differentiation has been found in invasive plants that have rapidly shifted growth and reproductive rates subsequent to introduction (Sexton et al. 2002; Siemann and Rogers 2003; Blair and Wolfe 2004; DeWalt and Hamrick 2004; Maron et al. 2004; Brown and Idris 2005). Selection has been found to act on plasticity in invasive populations in response to light and water availability (Zou et al. 2009), and after hybridization with native subspecies (Lavergne and Molofsky 2007). 
Though dissimilarities in habitat can result in genetic differentiation between populations in invasive and native ranges, divergence may be the result of non-adaptive processes such as genetic drift rather than selection (Bossdorf et al. 2005; Dlugosch and Parker 2008a; Kilkenny and Galloway 2013). The invasive bunchgrass Brachypodium sylvaticum (Hudson) Beauv., provides an ideal model for studies of adaptation during invasion, as a previous study with B. sylvaticum confirmed that trait divergence in morphological and physiological traits related to water uptake was the result of selection in the introduced range opposed to neutral evolutionary processes such as drift (Marchini and Cruzan unpublished). Here, we build on previous research finding that genetic differentiation is a product of selection to determine if trait values exhibit phenotypic plasticity, while also investigating the adaptive value of plasticity and genetic differentiation for individuals in the invaded range.

Brachypodium sylvaticum is an aggressive invader of the mixed-conifer understory habitats of the Pacific Northwest, USA, where it is capable of forming extensive monocultures. Brachypodium sylvaticum's invasion was facilitated by multiple introductions from across its native range, with invasive individuals mainly the result of hybridization among introduced populations from Western and Southern Europe (Rosenthal et al. 2008). Multiple introductions can provide a larger gene pool for selection to facilitate local adaptation and are often associated with invasive success (Riis et al. 2010).

Brachypodium sylvaticum displays broad climatic tolerances in its native range, occurring in arid, warm temperate, and boreal regions. Upon introduction to the invasive range, populations of $B$. sylvaticum had to acclimatize to the temperate Mediterranean 
climate of the Pacific Northwest USA, tolerating dramatic variations in seasonal rainfall and a long summer drought that contrasted to the relatively consistent precipitation patterns that are characteristic of the temperate Maritime regions in Western Europe. We hypothesized that the long summer drought of the invasive region would challenge introduced populations of B. sylvaticum as its primary growing season occurs during the warmest and driest part of the summer when it flowers and produces seed.

We chose six leaf and xylem morphological traits to test for genetic differentiation and plasticity in plants from native and invasive populations of $B$. sylvaticum in response to water limitation. We also measured two fitness-related traits, final size and shoot biomass, to investigate the possibility that plasticity and/or genetic differentiation is adaptive. Rapid growth and large size is thought to increase competitive ability and contribute to invasion success (Van Kleunen et al. 2010), and if adaptation to novel environments were occurring we would expect to find high values of these fitnessrelated traits in invasive individuals.

Our study addresses the hypothesis that plasticity is a mechanism aiding invasive success of $B$. sylvaticum. The plastic ability of plants from native and invasive populations of B. sylvaticum was measured as genotype-by-environment interactions (Via and Lande 1985) in one water-available and one water-limited treatment. We also explore an alternate hypothesis that selection has resulted in genetic differentiation of individuals from invasive populations. Because genetic differentiation can occur as a result of climate-driven selection, we include tests of abiotic environment on trait morphology. The adaptive potential of plasticity and differentiation was assessed with directional $(\beta)$ and quadratic $(Y)$ selection gradients (Lande and Arnold 1983), regressing trait values 
and plasticity across environments onto fitness-related traits (shoot biomass and final plant size). Because populations in the invasive range of $B$. sylvaticum consist of hybridized individuals from native source regions, we weighted our models by the probability of native population contribution to the invasive environment, determined by multilocus genotypes at microsatellite markers. If selection in the novel environment is driving invasive success, we would expect to find genetic differentiation between invasive and native populations of $B$. sylvaticum, instead of observing treatment-driven phenotypic differences. Utilization of selection gradients to test for selection on plasticity or genetic differentiation provides insight into the underlying drivers of adaptation in invasive species.

\section{MATERIALS AND METHODS}

Study system-Brachypodium sylvaticum (slender false brome) is a diploid perennial C-3 bunchgrass native to Europe, Asia, and Africa, with invasive populations in North America and Australia. The first herbarium specimen of B. sylvaticum was collected in 1939 in Eugene, Oregon (Ramakrishnan et al. 2008). B. sylvaticum maintained small populations in central Oregon until the 1980's, when it began to spread throughout central Oregon. Populations are now rapidly expanding, and invasive $B$. sylvaticum can be found in Washington, California, Virginia, New York, and British Columbia and Ontario, Canada (Miller et al. 2011). Invasive populations of B. sylvaticum show much variability in habitat, and can be found in areas ranging from wetland and riparian habitat to dry forests. Invasive populations form a monoculture carpeting the area and preventing establishment of native plants and tree seedlings. B. sylvaticum's recent 
spread, wide native range, and varied habitat make it an ideal model system for study of the evolutionary changes accompanying invasion.

Independent introductions of B. sylvaticum into Corvallis and Eugene, Oregon have been confirmed by microsatellite marker analysis (Ramakrishnan et al. 2008). Rosenthal 2008 also found that multiple introductions of B. sylvaticum from several regions throughout Western Europe (the United Kingdom, Greece, and Spain) had occurred and that these individuals hybridized with each other, resulting in their novel recombinant genotypes being found throughout the invasive range.

Native B. sylvaticum populations encompass a wide latitudinal distribution over several climatic regions. Seeds from a number of these areas, including the ones that will be used in this study, are available through the USDA's National Plant Germplasm System. The hybrid genotypes that are currently spreading throughout Oregon are a combination of populations from oceanic (United Kingdom), hot-summer Mediterranean (Greece), and cold semi-arid desert (Spain) climate types (Ramakrishnan et al. 2008). All of these climates represent a significant shift in precipitation from Oregon's cool-summer Mediterranean climate. The Oregon climate provides significantly less precipitation than in the UK, while significantly more than in Spain or Greece.

Greenhouse Methods- We performed a common garden study to assess plasticity and phenotypic differentiation of leaf morphological traits in response to water availability of the environment. Plants were grown in a research greenhouse due instead of in the field due to the ethical complications associated with growing an invasive species outdoors. Plants from thirty-three invasive and native populations of $B$. sylvaticum were grown in a common garden experiment from August 2012 to December 
$2012(\mathrm{n}=5$ individuals per population; see Appendix 1 for population sampling locations) Six study plots were established in $1 \mathrm{~m}^{3}$ raised bed plots in the research greenhouse at Portland State University, Portland, Oregon, USA. Boxes were filled with sandy loam soil ( $5 \%$ clay, $45 \%$ sand, and $50 \%$ silt). The six study plots were given $1 / 2 \mathrm{~L}$ of water after transplanting to acclimate the plants to the new growing conditions. Half of the plots were designated for drought treatment (watered minimally at first signs of wilting to mimic Oregon summer drought), while the other half were designated for regular watering (approximately $1 / 2 \mathrm{~L}$ a week to approximate natural rainfall patterns during Oregon's rainy season).

Brachypodium sylvaticum seeds were collected from 11 invasive populations located in Oregon, USA and 21 native populations located throughout Europe, North Africa, and the Middle East (Table A.D1). Seeds were obtained from the Western Regional Plant Introduction Station (Western Regional Plant Introduction Station, Pullman, WA, USA) and the Millennium Seed Bank Project (Kew, Surrey, UK). Seeds were planted in the glasshouse at Portland State University, Portland, OR, USA, and plants were maintained for a period of six months. Two tillers per plant were collected for use in the current experiment, with one tiller per individual placed in each of the waterlimited and water-available treatments.

Five tillers per population were each planted in water-limited and well-watered treatments, (with the exception of populations ESH, M3, M7, TUK, UK4, which had 4 tillers, and M16, which had 3 tillers) representing different individuals from each population of B. sylvaticum. Tillers were randomly placed in each raised bed at a fixed distance of $10 \mathrm{~cm}$ from one another. Water treatment was induced after the plants had 
been allowed to grow and establish for one month, and was carried out for approximately five months. At the end of the five-month growing period average plant height and tiller number data were collected to quantify population fitness and growth. Because $B$. sylvaticum exhibits growth vertically and through tiller production, we define plant size as the product of height and tiller number prior to harvest. Plants were harvested five months after planting and shoot biomass was obtained by placing plants in convection drying ovens $\left(70^{\circ} \mathrm{C}\right)$ until a constant mass was achieved (minimum of 48 hours). We were not able to acquire root biomass as roots become tangled after long growth periods, making them difficult to pull apart without significant loss.

Morphological Variables Measured- We measured six morphological traits related to water uptake in plants: xylem vessel frequency $(V F)$, maximum xylem vessel diameter $(M V D)$, hydraulically weighted mean xylem vessel diameter $(H M D)$, bulliform cell area $(B A)$, stomatal index $(S I)$, and specific leaf area $(S L A)$. In water-limited conditions, xylem vessel frequency $(V F)$ is expected to increase while vessel diameter ( $M V D$ and $H M D$ ) decreases, resulting in xylem vessels that are less prone to cavitation while maintaining water uptake (Tyree and Zimmermann 2002; Tombesi et al. 2010; Smith et al. 2013). Bulliform cells control leaf rolling, a behavior that reduces surface area thus evaporation off the leaf surface; a smaller bulliform cell area $(B A)$ allows more rapid loss of turgor, promoting more rapid rolling of leaves in water-limited environments (Kadioglu et al. 2012). Stomatal index (SI) is a normalized measure of stomatal density across a leaf's surface; many, small stomata are expected to reduce water loss in drought compared to larger, less frequent stomata (Beerling and Chaloner 1992; Xu and Zhou 2008). Specific leaf area (SLA), the ratio of leaf surface area to 
mass, is expected to decrease in drought as leaves become thicker to reduce water losses (Larcher 1995; Poorter et al. 2009). See Chapter 3, Table 3.2 for trait details.

One most recently expanded leaf was collected from each individual for $S I$ and SLA measurements. Approximately $2 \mathrm{~cm}$ of tissue was collected from the base of each leaf and placed in $2.0 \mathrm{~mL}$ vials filled with a 200:5 50\% ethanol-glacial acetic acid $(17.5 \mathrm{M})$ mixture to preserve tissue for analysis of metaxylem characteristics $(V F, M V D$, $H W M D)$. Due to differences in the growth rates of the plants and size limitations, leaves used for tissue samples were collected in two separate batches: half at the end of November and half at the end of December. During each sampling session, matching tillers from each individual were always collected in clonal pairs (one plant from each treatment) and a range of populations was gathered to avoid biased sampling of populations. Leaves collected in the greenhouse were kept in water and processed within the same day.

Stomatal and metaxylem counts and measurements were made using a Leica MZ16 stereomicroscope (Leica Microsystems, Wetzlar, Germany) linked to a QImaging Retiga 1300 camera (Q-Imaging, Surrey, British Columbia, Canada) and analyzed with Image-Pro 6.0 Software (Media Cybernetics, Warrendale, PA, USA). Leaf epidermal impressions were obtained according to methods in Beerling and Chaloner (1992) for calculation of SI [stomatal density/(stomatal density + epidermal cell density) x 100, where stomata consist of the stomatal pore and two flanking guard cells], a measurement that normalizes leaf stomatal density in relation to the confounding influence of epidermal cell expansion that may be initiated by factors other than those influencing stomatal development (Royer 2001; Xu and Zhou 2008). 
Cross sections of preserved leaf samples were photographed and measured for metaxylem and bulliform cell characteristics on at least 3 individuals per population. Images were analyzed with ImageJ (National Institutes of Health, Bethesda, Maryland, USA). Measurements on each section included cross-sectional area, area of the major veins, and proportion of bulliform cells and vascular tissue to cross-sectional area. These measurements were utilized to obtain $H M D, V F, M V D$, and $B A$ for each individual. To assess average metaxylem diameter, the principles of the Hagen- Poiseuille relationship, which state that a conduit's hydraulic conductivity is proportional to its diameter to the $4^{\text {th }}$ power, were taken into account. Thus, to find $H M D$, raw measurements of diameter were analyzed for their frequency in $1 \mu \mathrm{m}$ size classes and for the relative contribution of each class of diameter to the sum of all the conduits raised to the $4^{\text {th }}$ power calculated as: $H M D=2 \frac{\Sigma \mathrm{r}^{5}}{\Sigma \mathrm{r}^{4}}$, where $\mathrm{r}$ is the radius of a xylem conduit. This equation weights the importance of radii in proportion to the estimated hydraulic conductance of the xylem conduits (Sperry and Saliendra 1994). $V F$ was calculated as the average number of metaxylem vessels per leaf area. $M V D$ is the largest metaxylem vessel per individual. The cross-sectional area of bulliform cells, large leaf surface cells that are the first to lose turgor in times of water stress, resulting in leaves rolling in to prevent transpirational water loss, was found for each individual. $B A$ was calculated as the proportion of bulliform cells present per cross-sectional area.

To obtain $S L A$, the surface area for each plant was measured by first photographing the top half of the most recently fully expanded leaf using a Kaiser RA-1 copy stand with a Panasonic WV-BD400 camera attachment. The surface area of this portion of the leaf was estimated using the drawing tool in Image J. The top half of each 
leaf was gathered and dried in an oven for at least 48 hours at $60 \mathrm{C}$ to constant weight. $S L A$ was calculated as leaf surface area in $\mathrm{m}^{2} /$ leaf dry weight in $\mathrm{kg}$ for each plant.

Statistical Analysis Weights of Genetic Contribution-Prior to performing statistical analysis we weighted traits of native individuals by probability of contribution to invasive populations. Assignment tests of the probability of contribution of native to invasive populations were performed using the genotypes of Brachypodium sylvaticum plants at eight microsatellite loci with primers developed and optimized at Portland State University (Ramakrishnan et al. 2008a). Primers are deposited at GenBank (Accession nos EF450748, EF450751, EF450752, EF450754, EF450756, EF450757, EF450759, EF450765). Assignment tests were performed in Geneclass2 utilizing the Rannala and Mountain (1997) assignment method of identifying immigrants based on their multilocus genotypes to detect immigrant ancestry (Rannala and Mountain 1997; Piry et al. 2004). The assignment probabilities were pooled across invasive populations, to create an average probability of genetic contribution of each native population to the invasive range. Mixed linear regressions were then weighted by this genetic probability of contribution.

Phenotypic Plasticity and Genetic Differentiation- We used restricted maximum likelihood models with random effects to determine plasticity and trait divergence across water-limited and water available treatments (lmer and lmerTest, lme4 package; R Core Team 2013). To account for phenotypic variation based on climate of source population, each model included a composite abiotic environmental covariate determined by a principal components analysis (PCA) of latitude and six climatic variables (aridity, potential evapotranspiration, temperature, evaporation, precipitation, and cloud cover). 
Climatic data for Oregon, USA populations were obtained from the Prism Group, Oregon State University (PRISM Group 2004); climatic data for native source populations located throughout Europe, the Middle East, and Asia were obtained using WorldClim (Hijmans et al. 2005) and United State Geological Climate Survey (USGS) climate data (Hearn et al. 2003). The principal component PC1 explained most variation across regions, and was utilized in all trait models as the environment term.

Water treatment, region (invasive or native), environment (PC1), the interaction of environment $\mathrm{x}$ region, and the interaction of treatment $\mathrm{x}$ region were treated as fixed factors in the model; treatment nested within raised bed was a random effect. A significant effect of region in these models would indicate differentiation in the given trait between plants from invasive and native regions; a significant effect of treatment would indicate plasticity for the given trait. All response variables were $\log x+1$ transformed to meet assumptions of normality for regression.

Plasticity and Selection Gradients- For all selection analyses we utilized standardized explanatory values (mean $=0, \mathrm{SD}=1$ ) to allow comparison between regression coefficients of traits measured on different scales (Van Kleunen 2001). The relative fitness of shoot biomass and final plant size was calculated for response variables. Response variables were $\log x+1$ transformed and also standardized to mean $=0$ $\mathrm{SD}=1$. All models were weighted by the probability of contribution of native to invasive ranges.

Selection on plasticity for traits across water-available and water-limited treatments was tested using linear regression (Weis and Gorman 1990). The standardized values of absolute plasticity (mean of treatment 1 minus mean of treatment 2) and 
standardized mean trait values were explanatory variables in the model. Response variables were the fitness related traits of shoot biomass and final plant size. A positive effect of phenotypic plasticity on a fitness related trait is indicated by a positive regression coefficient for a trait, while a negative selection coefficient suggests selection against plasticity (Van Kleunen and Fischer 2001).

We estimated linear $(\beta)$ and univariate nonlinear (quadratic, $\gamma$ ) selection for morphological traits in water-limited and water-available environments (Lande and Arnold 1983). Selection models were weighted by the probability of genetic contribution of native to invasive populations (Marchini and Cruzan unpublished). Directional selection gradients were estimated using linear models regressing standardized trait values onto the standardized relative fitness values for shoot biomass and final size (lmer; $\mathrm{R}$ Core Group 2013). The linear selection coefficient $\beta$ is found as the partial regression coefficient for each morphological trait. Significance of linear selection coefficients is indicative of directional selection for higher (positive $\beta$ ) or lower (negative $\beta$ ) phenotypic values that effect fitness-related traits. Nonlinear models included all linear and quadratic values of standardized traits and their interaction terms. The partial regression coefficient of the quadratic terms was the quadratic selection coefficient. Quadratic selection gradients were doubled (Stinchcombe et al. 2008). Stabilizing selection is revealed by a concave quadratic term, while disruptive selection is indicated by a convex quadratic term.

\section{RESULTS}


Principal Components Analysis of Climate- Results from the PCA of population climatic characteristics show that PC1 and PC2 explained $50 \%$ and $19 \%$ of the variance across populations, respectively. Axis 1 was correlated with aridity and latitude (a smaller value for aridity indicates a drier environment); Axis 2 was correlated with surface evaporation and precipitation. Although there is overlap in the ellipses of climatic variation between native and invasive source regions, invasive regions are more strongly influenced by aridity (PC1; Fig. 5.1).

Plasticity and Genetic Differentiation- Probabilities of contribution used in analyses are found in Appendix D, Fig. D1. There was no effect of treatment or a treatment $\mathrm{x}$ region interaction for any measured traits, indicating a lack of plasticity. There was significant genetic differentiation between ranges of three traits related to xylem morphology $H M D, M V D, V F$, and one trait related to cell size, $B A$ (Fig. 5.1, Table 5.1). $H M D$ and $M V D$ were significantly lower in invasive populations than in native populations, while $V F$ was higher in invasive populations than native populations, indicating that plants in invasive populations possess smaller, more frequent xylem vessels than plants from native populations. The expression of four traits, $V F, H M D$, $M V D$, and $B A$ in individuals from the invasive range conformed to hypotheses of optimal trait values expected in water-limited environments (Fig.5.2, Fig. 5.3).

Environment was associated with values of $H M D, M V D$, and $B A$ (Table 5.1, Fig. 5.4a, $\mathrm{b}, \mathrm{c})$, and although trait values for plants from the invasive range appeared to be associated with lower PC1 scores (more arid environment), the environment $\mathrm{x}$ region interaction term was not significant for these variables. The environment $\mathrm{x}$ region 
interaction was significant for SI, with invasive range values more closely correlated with lower PC1 values (Fig. 5.4d).

Plasticity, Linear, and Quadratic Selection Gradients- The majority of linear selection coefficients for plasticity were negative (Table 5.3). There was a significant negative selection coefficient for plasticity in $V F$ on shoot biomass (Table 5.2), indicating that greater plasticity in $V F$ across water-limited and water-available treatments is associated with a lower shoot biomass (Figure 5). No other traits had significant selection coefficients of plasticity.

Four out of six traits had significant directional selection gradients for shoot biomass and final size in the water-limited treatments (Table 5.3). Gradients ran in the same direction for shoot biomass and final size in response to all traits. A negative selection coefficient for $V F$ describes reduced aboveground size with an increasing frequency of xylem vessels. The selection gradient of $M V D$ is positive, indicating that possession of at least one large xylem vessel results in increased size and biomass, although the negative coefficient of $H M D$ indicates selection towards a smaller size of xylem vessels. Negative selection gradients for $B A$ imply negative selection for increasing bulliform cell area in water-limited environments.

In the water available treatment, the majority of linear selection coefficients were negative, although none were statistically significant (Table 5.3). There was a significant negative quadratic term for $V F$ (Fig. 5.6a), and a positive quadratic term for $B A$ (Fig 5.6b). Inspection of these curves show that the quadratic functions are mostly linear.

Genetic Differentiation and Direction of Selection- Of the four traits found to be significantly different across invasive and native ranges of B. sylvaticum, the direction of 
selection for greater relative fitness was only consistent with the direction of phenotypic differentiation occurring in the invasive region for two traits in the water limited treatment. One of these traits is related to xylem morphology $(H M D)$, and one of these traits is related to cell morphology $(B A)$. Both $H M D$ and $B A$ were associated with negative selection gradients, consistent with the smaller values of $H M D$ and $B A$ found for individuals from invasive populations (Table 5.3, Fig. 5.2).

Two of the four traits that significantly differed across invasive and native ranges of $B$. sylvaticum displayed trait values inconsistent with the direction of selection for relative fitness of shoot biomass and final size in the water limited treatment. These traits, $V F$ and $M V D$, are both related to xylem vessel morphology. A negative selection coefficient implies greater relative fitness with decreasing frequency of xylem vessels, in contrast to the greater $V F$ found in plants from invasive populations. The positive selection coefficient for $M V D$ suggests greater relative fitness with a larger maximum diameter of the xylem vessels, while plants from invasive populations possess smaller MVD than their native counterparts.

\section{DISCUSSION}

Our study found that invasion success in Brachypodium sylvaticum has been facilitated by post-introduction selection leading to genetic differentiation rather than by plasticity. This result contrasts with the hypothesis that plasticity has allowed invasive individuals the flexibility to establish in multiple habitats. Our results instead show that two out of four measured traits exhibiting genetic divergence were adaptive in a water- 
limited environment, while one trait displayed local adaptation to the abiotic environment in the invasive range.

Plasticity- There were no significant effects of treatments on trait expression indicating a lack of plasticity in response to contrasting water environments for plants from both invasive and native ranges of $B$. sylvaticum. These results are contrary to findings that plasticity is high in populations of invasive species (Davidson et al. 2011), but in line with other studies concluding that there are no differences in plasticity between invasive and native populations (Godoy et al. 2011; Palacio-López and Gianoli 2011). Moreover, selection gradients for aboveground biomass were negatively correlated with plasticity for the frequency of xylem vessels in leaves (VF; Fig. 5), indicating selection against plasticity for this trait.

Contradictory reviews have found that plasticity either plays a major (Davidson et al. 2011), or very minor role (Godoy et al. 2011; Palacio-López and Gianoli 2011) in the invasive process. A recent hypothesis proposed by (Lande 2015) provides an explanation for the inconsistent findings. Lande describes how introduction to a novel habitat can spur a rapid increase in plasticity during the lag phase, allowing individuals to approach phenotypic optima. This peak in plastic ability is followed by slow genetic assimilation of the new phenotypes into invasive populations (Lande 2015). Plasticity is reduced as adjustment to the novel habitat proceeds, barring frequent environmental perturbations (Lande 2009, 2015).Alternatively, the variation necessary for adaptive responses in the novel environment can be generated by admixture due to interbreeding among genotypes from multiple sources in the native range. However, due to the ephemeral nature of 
plasticity during the lag phase, this hypothesis is difficult to test on a species that has already become invasive.

Results show selection against plasticity in $V F$, indicating that plasticity may constrain rather than facilitate adaptation in the new environment. Generally, plasticity is thought to be favored in fluctuating environments and disadvantageous in homogeneous environments. Costs of plasticity are indicated by a reduction in fitness of a plastic compared to a fixed organism in the same environment (DeWitt et al. 1998). A cost of plasticity occurs when variation in phenotypic expression results in a fitness deficit, as a consequence of physical, developmental, and genetic constraints (DeWitt et al. 1998; Callahan et al. 2008). Similarly, plasticity can be maladaptive if the plastic response moves trait values away from phenotype optima for an environment. A cost of plasticity is implied by $V F$ plasticity resulting in smaller aboveground size and biomass.

Phenotypic Differentiation and Selection- Differences in selection gradients in the water-available and water-limited environments, combined with evidence that environmental variation at population locations influences trait values, suggests that moisture availability is a selective pressure driving genetic differentiation in the invasive range of B. sylvaticum. Trait shifts predicted to occur in drought were supported by invasive trait values; however, these traits shifts did not consistently result in greater aboveground biomass and size.

Two traits, $H M D$ and $B A$, displayed genetic divergence in the invasive range consistent with the direction of improved drought tolerance. Selection for reduced bulliform cell area in the invasive range translates into more rapid leaf rolling in response to moisture stress. Leaf rolling decreases leaf surface area, reducing transpirational 
losses, and is a common response to low water availability in drought tolerant species (O'Toole and Cruz 1980; Turner 1986; Kadioglu et al. 2012). Reduced xylem diameter decreases susceptibility to cavitation under drought stress (Hacke and Sperry 2001; Tyree and Zimmermann 2002; Tombesi et al. 2010). Genetic differentiation of these traits in the invasive range has likely facilitated by population persistence of $B$. sylvaticum during summer droughts in Oregon.

We found seemingly maladaptive genetic differentiation in the water-limited treatment for two traits related to xylem morphology $(V F$ and $M V D)$ in the invasive range of $B$. sylvaticum. Frequency of xylem vessels is related to xylem diameter; as diameters decrease, reducing the threat of cavitation, the frequency of vessels throughout the stem should increase so that water uptake can be maintained (Tyree and Zimmermann 2002; Smith et al. 2013). Similar to expectations of $H M D$ in drought, $M V D$ is predicted to decrease, lowering the probability of cavitation (Tyree and Zimmermann 2002); although it is possible that the advantage of maintaining several large xylem vessels permitting rapid water uptake outweighs cavitation risks. Despite these predictions, we found increased aboveground size and biomass with less frequent xylem and larger maximum xylem diameter.

Trade-offs between growth and resource allocation and tolerance to environmental stress may provide insight into the apparent contradiction between the direction of selection and observed patterns of differentiation for $V F$ and $M V D$. Grime (1977) suggested that plants could not be both highly competitive and highly tolerant to environmental stress. The existence of such trade-offs has been supported by recent studies finding that increased tolerance to abiotic stress often comes at a cost of reduced 
competitive ability (Petrů et al. 2006; Sambatti and Rice 2007; Liancourt and Tielbörger 2009; Hodgins and Rieseberg 2011). Distinct abiotic environments in the invasive and native range are likely driving selection and increasing invasive drought tolerance, while limiting aboveground growth potential.

A second explanation for inconsistency between the direction of selection and divergence is the possibility that fitness and competitive ability of $B$. sylvaticum is not driven by aboveground size and biomass. Though there are few studies finding that increased aboveground size is not a trait contributing to invasiveness (Willis et al. 2000a; Thébaud and Simberloff 2001); there are a bounty of studies finding that resource allocation to improve fitness in water-limited environments is often directed belowground (Chaves et al. 2003; Farooq et al. 2009). For example, a study of invasive and noninvasive woody species grown in a summer drought found that invasive species had much larger root masses than non-invasive species (Grotkopp and Rejmánek 2007). While requiring further investigation and speculative based on the scope of the current study, selection on B. sylvaticum individuals in water-limited environments may result in resource allocation and increased competitiveness belowground.

Effect of Environment- Abiotic environment was correlated with two traits related to xylem vessel size ( $M V D$ and $H M D$ ) and one trait related to cell morphology $(B A)$. There was a significant environment $\mathrm{x}$ range interaction for $S L A$ indicating that this trait for plants from the native and invasive ranges responded differently to changes in water availability. The sensitivity of these traits to environmental gradients suggests environmental specialization (Richards et al 2006). These results suggest local adaptation to environmental variability that has shaped genetic divergence between ranges and has 
led to evolution after introduction and establishment (Callaway and Maron 2006; Colautti et al. 2010).

Invasive populations of $B$. sylvaticum are composed of intra-specific recombinant hybrid genotypes as a result of multiple introductions (Rosenthal et al. 2008b). Invasive hybridization may be a factor promoting local adaptation of $B$. sylvaticum to the abiotic environment of the invaded region. Evidence of hybridization is commonly found in populations of invasive species (Ellstrand and Schierenbeck 2000; Gaskin and Schaal 2002; Zalapa et al. 2010; Kolbe et al. 2012). In B. sylvaticum, hybridization and multiple introductions have reduced genetic bottlenecks at sites of primary introduction (Rosenthal et al. 2008b). These primary introduction sites now act as sources for gene flow to satellite populations (Ramakrishnan et al. 2010a; Marchini and Cruzan unpublished), and likely provide the genetic variation necessary for strong selection to evolve phenotypes that are adapted to the local climatic conditions in the invasive range.

Conclusions- We have presented evidence that genetic differentiation and local adaptation rather than plasticity are drivers of invasive success in Brachypodium sylvaticum. Individuals from the invasive and native ranges of $B$. sylvaticum were not phenotypically plastic in environments of contrasting water availability, supporting studies concluding that plasticity is not a trait inherent to invasive species. We found genetic divergence between invasive and native ranges for four out of six traits measured. Differentiation of these traits was in the direction hypothesized to occur in response to drought stress in the introduced range. Divergence of invasive plants was not consistently in the direction indicated by selection gradients, implying that there are limitations of selection that may be a consequence of physical constraints and/or tradeoffs between 
growth and abiotic tolerance. Significant relationships between genetic divergence and environment suggest local adaptation of $B$. sylvaticum to local environmental conditions.

Future work on B. sylvaticum and other invasive plants will further explore the role of genetic variation resulting from hybridization and plasticity for processes of local adaptation leading to invasion success in novel environments. 


\section{Tables and Figures}

Table 5.1 P-values from mixed linear regressions finding the effects of range, abiotic environment, and treatment on 6 measured traits in B. sylvaticum.

\begin{tabular}{|c|c|c|c|c|c|c|c|c|}
\hline & & & $V F$ & $H M D$ & $M V D$ & $B A$ & $S L A$ & $S I$ \\
\hline Effect & Source & df & $P$ & $P$ & $P$ & $P$ & $P$ & $P$ \\
\hline \multirow[t]{6}{*}{ Fixed } & Treatment & 1 & 0.75 & 0.92 & 0.93 & 0.68 & 0.83 & 0.81 \\
\hline & Environment & 1 & 0.05 & $0.04 *$ & $0.04 *$ & $<0.01 * *$ & 0.19 & 0.26 \\
\hline & Range & 1 & $0.04 *$ & $0.02 *$ & $0.07 *$ & $<0.01 * *$ & 0.42 & 0.93 \\
\hline & Environment $\mathrm{x}$ Range & 1 & 0.50 & 0.41 & 0.53 & 0.20 & 0.30 & $<0.01 * *$ \\
\hline & Treatment $\mathrm{x}$ Range & 1 & 0.48 & 0.46 & 0.49 & 0.30 & 0.26 & 0.48 \\
\hline & & & Variance & Variance & Variance & Variance & Variance & Variance \\
\hline Random & Raised Bed (Treatment & & 0.32 & 0.003 & 0.001 & 0 & 27.41 & 2.57 \\
\hline
\end{tabular}


Table 5.2 Directional $(\beta)$ and quadratic $(\gamma)$ selection gradients on final size and shoot biomass of invasive and native populations of Brachypodium sylvaticum. The table lists also lists standardized regression coefficients of plasticity for final size and shoot biomass. Asterisks indicate statistical significance of regression coefficients: $*=p<0.05, * *=p<0.01$. The presented quadratic gradients are twice the estimated partial regression coefficients.

\begin{tabular}{|c|c|c|c|c|}
\hline \multirow[t]{3}{*}{ Value } & \multicolumn{4}{|c|}{ Final Size } \\
\hline & \multicolumn{2}{|c|}{ Water-available } & \multicolumn{2}{|c|}{ Water-limited } \\
\hline & $\beta$ & $r$ & $\beta$ & $r$ \\
\hline$V F$ & -0.2078 & 0.427 & $-1.215 * *$ & 0.396 \\
\hline$M V D$ & -2.8161 & -3.798 & $3.116 *$ & -3.1 \\
\hline$H M D$ & 3.0408 & 3.897 & $-3.175 *$ & 2.614 \\
\hline$S I$ & 0.3524 & 0.389 & -0.466 & -0.468 \\
\hline$S L A$ & -0.9065 & 3.743 & 0.166 & 0.054 \\
\hline \multirow[t]{4}{*}{$B A$} & -0.3966 & -3.039 & $-0.436 *$ & -0.076 \\
\hline & \multicolumn{4}{|c|}{ Shoot Biomass } \\
\hline & \multicolumn{2}{|c|}{ Water-available } & \multicolumn{2}{|c|}{ Water-limited } \\
\hline & $\beta$ & $r$ & $\beta$ & $r$ \\
\hline$V F$ & -0.4853 & $0.794 * *$ & $-1.076 * *$ & 0.268 \\
\hline$M V D$ & -2.1455 & -2.22 & $4.031 * *$ & 1.178 \\
\hline$H M D$ & 1.8938 & 2.884 & $-4.326 * *$ & -1.038 \\
\hline$S I$ & 0.1366 & -0.052 & -0.133 & -0.036 \\
\hline$S L A$ & -0.1358 & 1.564 & 0.331 & 0.292 \\
\hline$B A$ & -0.2212 & $-2.782 * *$ & $-0.331 *$ & -0.252 \\
\hline
\end{tabular}


Table 5.3 Directional $(\beta)$ selection gradients of plasticity in six traits on final size and shoot biomass of invasive and native populations of Brachypodium sylvaticum. Asterisks indicate statistical significance of regression coefficients: ${ }^{*}=\mathrm{p}<0.05$.

\begin{tabular}{ccc}
\hline \hline Plasticity & Final Size & Shoot Biomass \\
\hline$V F$ & -0.359 & $\mathbf{- 0 . 7 8 9 *}$ \\
$M V D$ & -4.078 & -2.997 \\
$H M D$ & 3.854 & 2.255 \\
$S I$ & 0.017 & 0.211 \\
$S L A$ & -0.105 & 0.083 \\
$B A$ & -0.607 & -0.594 \\
\hline \hline
\end{tabular}


Figure 5.1 Principal components analysis biplot of variables associated with climate in populations of Brachypodium sylvaticum. Points represent range of Brachypodium sylvaticum populations (I=Invasive, $\mathrm{N}=$ Native).

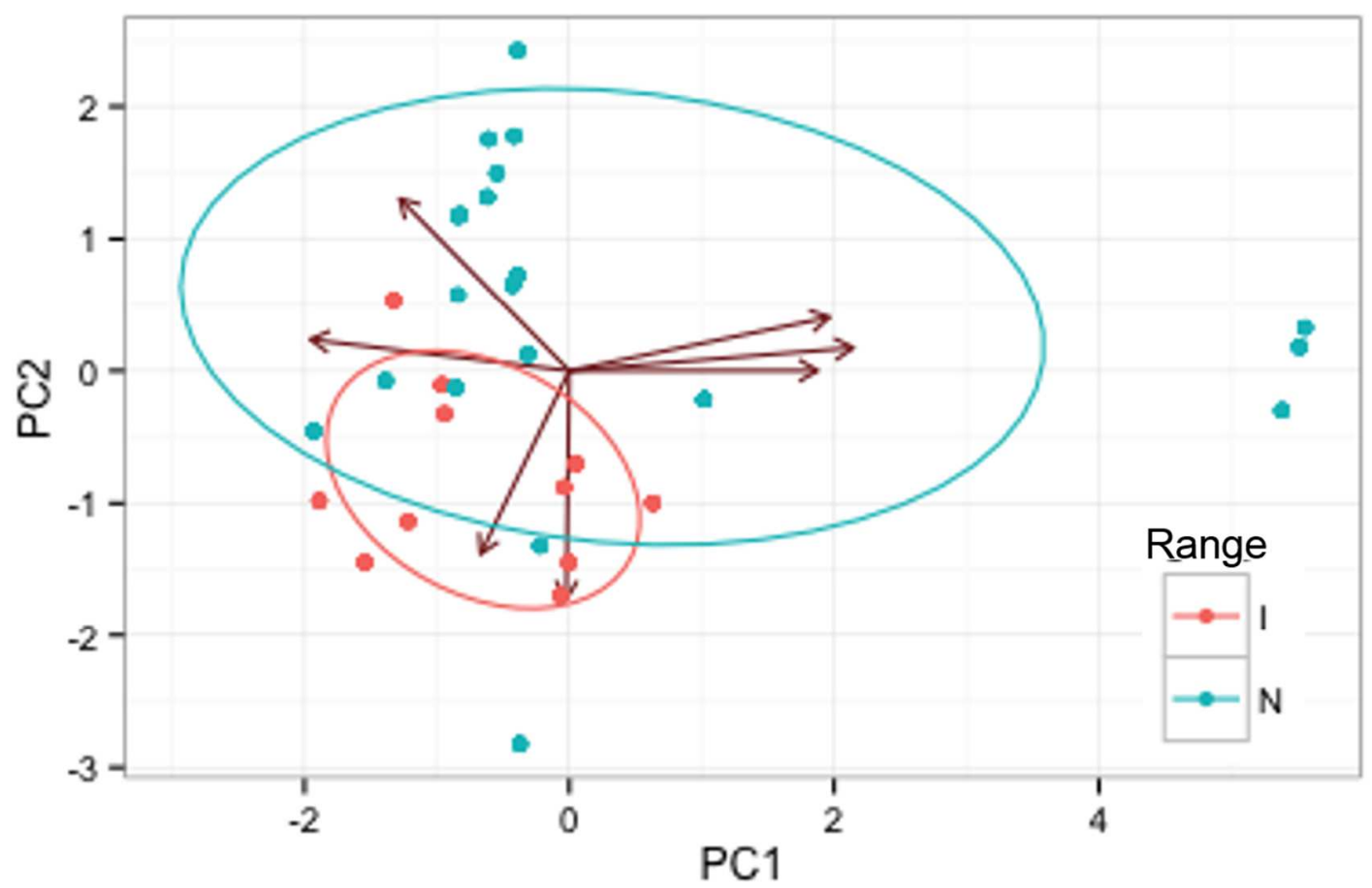


Figure 5.2 Trait means for a) VF, b) MVD, c) HMD, d) SI, e) SLA, and f) BA in individuals from invasive and native range of Brachypodium sylvaticum. Arrows indicate direction of trait shifts under drought stress. Data is weighted by the probability of genetic contribution found by multilocus comparisons. Asterisks indicate statistical difference of trait values between regions: $*=p<0.05$.
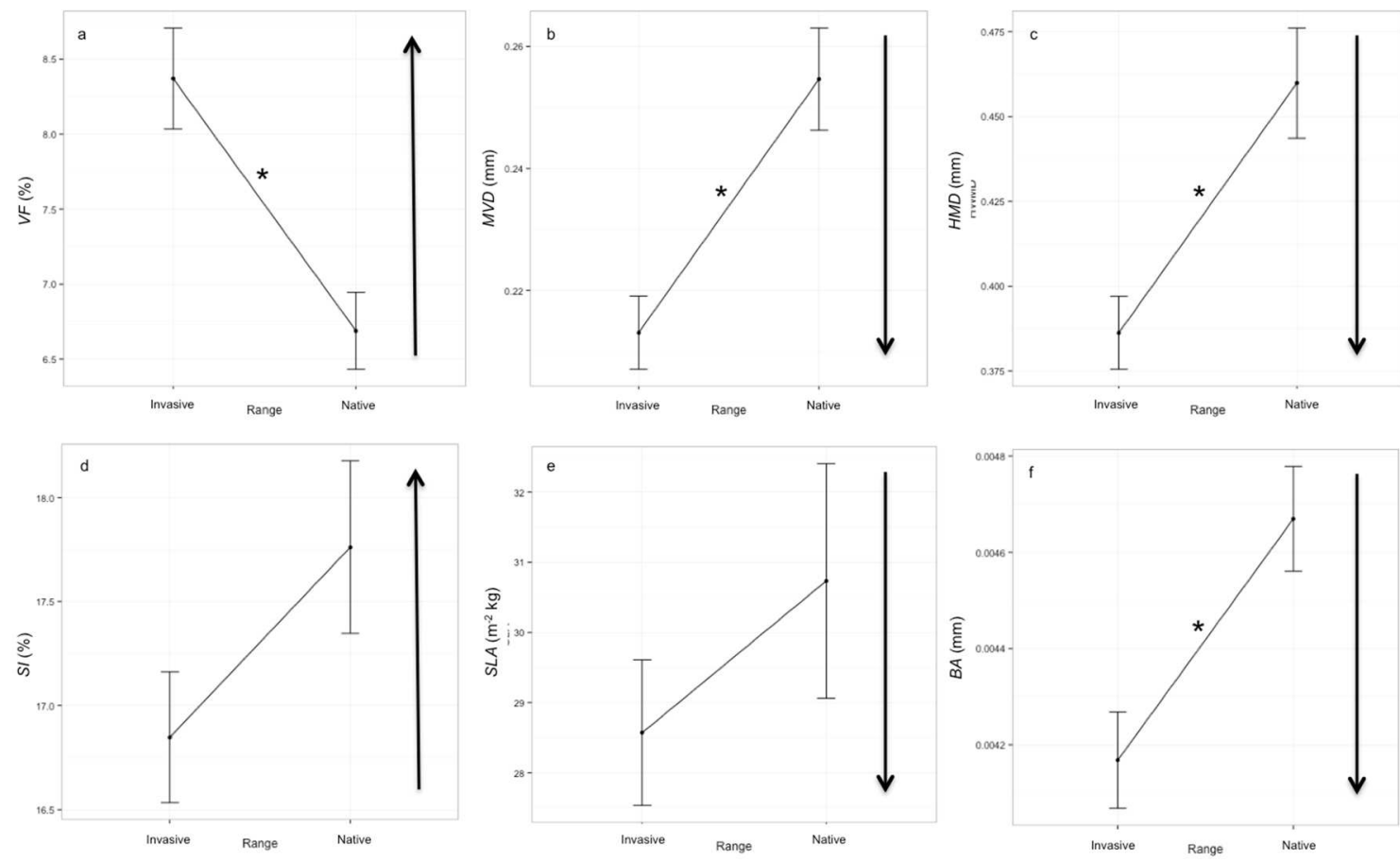
Figure 5.3 Examples of Brachypodium sylvaticum leaves from invasive (Corvallis, Oregon, USA, left) and native (Larissa, Greece, right) ranges. a) Cross-sections of leaves. Invasive plants have smaller bulliform cells and smaller, more frequent metaxylem vessels. b) Surface imprints of the leaf epidermis. Invasive individuals have lower stomatal density.

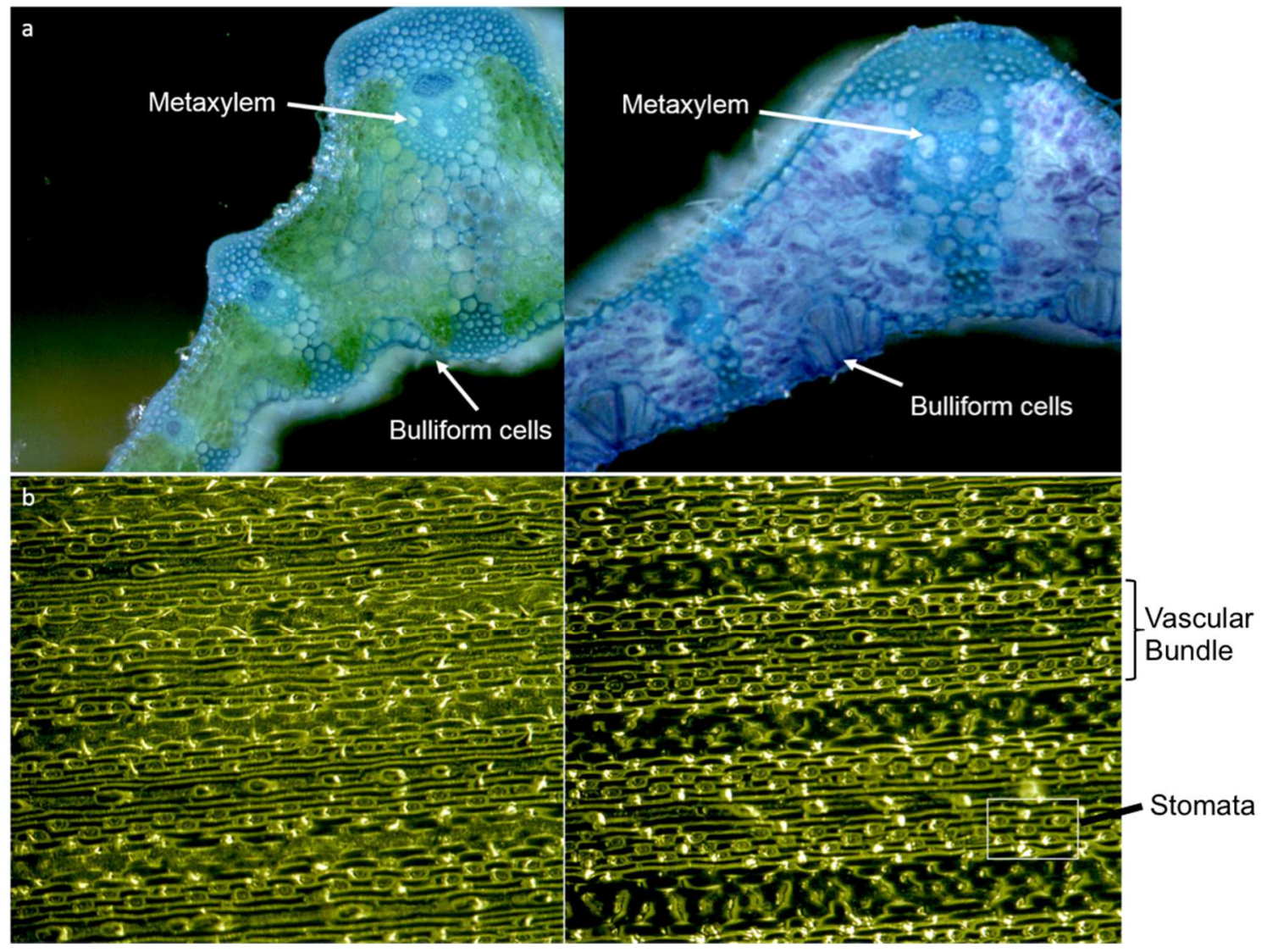


Figure 5.4. Environmental gradients and phenotypic expression for four morphological traits of Brachypodium sylvaticum. a) Maximum vessel density, b) Hydraulically weighted mean diameter, c) Bulliform cell area, and d) An interaction of range and abiotic environment in for stomatal index. PC1 is strongly correlated with aridity. Error bars are $\pm \mathrm{SE}$.
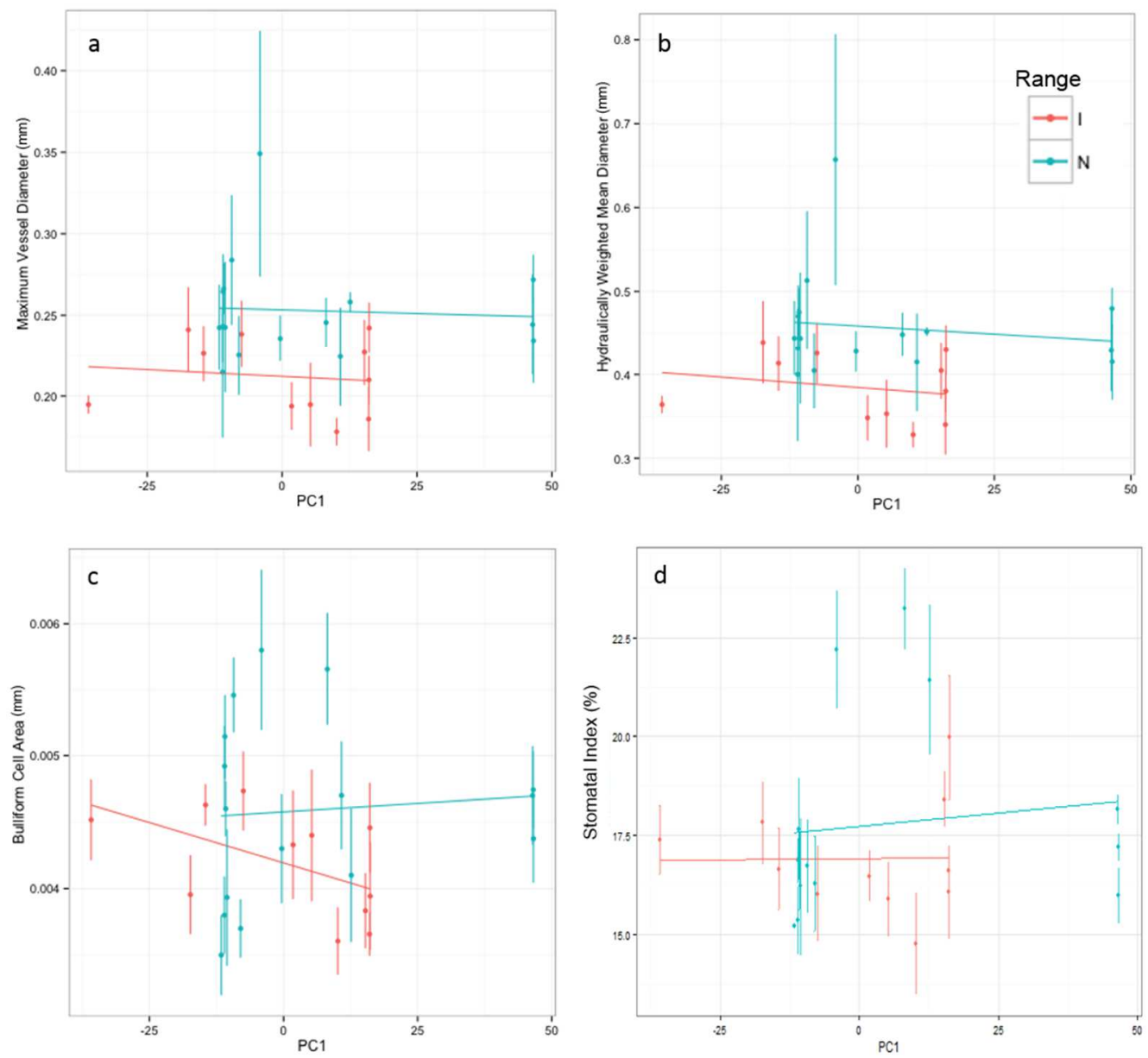
Figure 5.5 Significant negative directional selection $(\beta)$ for $V F$ plasticity in response to water availability in Brachypodium sylvaticum. Plasticity is measured as mean values of treatment 1- treatment 2 per population.

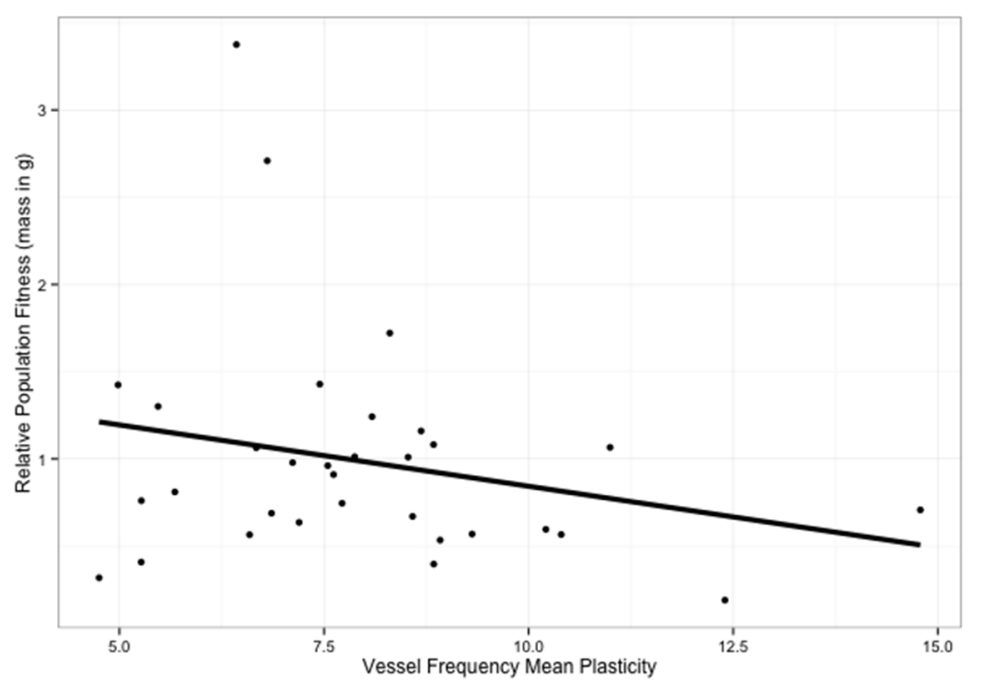


Figure 5.6 Quadratic selection $(\Upsilon)$ for a) $V F$ and b) $B A$ in the water available treatment. Selection curves are significant at $\mathrm{p}<0.05$.
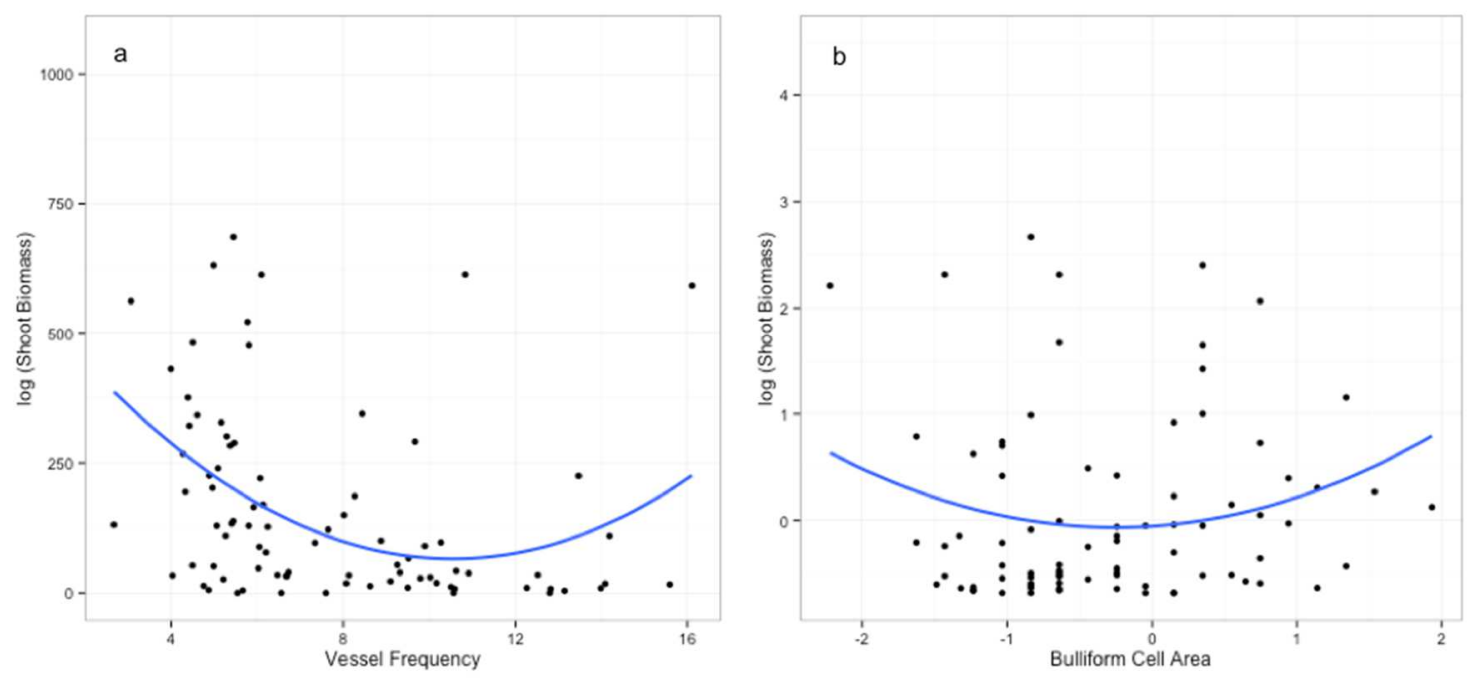


\section{Chapter 6}

\section{Conclusions: Management Implications of Studies}

The combined works of this dissertation significantly contribute to the field of invasion biology by uncovering mechanisms underlying the establishment success of introduced species. While it is clear that these studies have allowed development of hypotheses of evolutionary mechanisms underlying invasion in Brachypodium sylvaticum, these results also have a practical application for management practices to limit invasive plant spread.

In chapter two, I establish that purging of genetic load is facilitated by intermittent gene flow and that genetic purging increases the success of populations with low genetic diversity $\left(H_{\mathrm{S}}\right)$ and effective population size $\left(N_{\mathrm{e}}\right)$. This scenario of genetic purging is also plausible for other plants, and thus results are applicable for management practices of species other than B. sylvaticum. While B. sylvaticum's ability to self-fertilize promotes its' potential to purge genetic load, I hypothesize that without self-fertilization, it is possible to purge genetic load through close-kin inbreeding that would occur in populations with low $N_{\mathrm{e}}$. As genetic purging in a population with small $N_{\mathrm{e}}$ is facilitated by intermittent gene flow from high $H_{\mathrm{S}}$ populations, management practices should focus on centrally located populations near the sites of primary invasion. By destroying highly fit central populations, managers can cut off gene flow from these populations to the range edge, increasing the detrimental effects of bottlenecks and slowing invasion.

Evidence for genetic purging in populations of B. sylvaticum was expanded on in chapter three, where I discuss how reductions in fitness due to the effects of low effective population size $\left(N_{\mathrm{e}}\right)$ can be mitigated by stress reduction; specifically that nitrogen 
deposition may be increasing the chance that low $H_{\mathrm{S}}$ populations of invasive species are spreading. These results point towards the process of genetic purging being environmentally dependent, as fitness of individuals from low genetic diversity populations was only equal to the fitness of individuals from high genetic diversity populations in a high nitrogen environment. This suggests that invasive control should be focused on populations where environmental stress is likely to be low. The low-stress areas include habitats subject to high nitrogen deposition and runoff, and may be broadened to include environments where frequent physical disturbance limits competition.

In chapter 4, I found evidence of selective processes occurring during the establishment phase of invasion, as well as developed a robust method for assessing phenotypic divergence in species that are invasive or expanding their range. This study points towards the result that phenotypic adaptation in invasive species occurs prior to invasive range expansion. Once a species is introduced, selective process take place prior to invasive spread. This study has a more difficult implication for management, as an introduced species must be eradicated prior to invasion, and most management practices only begin control after invasive spread. The main lesson from this study is that management practices should focus on newly invasive species, as it is impossible to know how detrimental any new introduced species may become. After a species becomes invasive and overcomes secondary bottlenecks hindering range expansion, control becomes increasingly difficult if not impossible.

In chapter 5, I determine that trait differentiation is more important than plasticity in invasive $B$. sylvaticum, and find evidence that plant growth may be constrained by 
selection for traits tolerant to the abiotic environment. These results build on evidence of selection from chapter 4, and show that a plant's size may not be representative of its adaptation to the invaded environment, as a drought tolerant plant may not be the largest. This result implies that invasive practices should not just be focused on the largest individuals in a population, but that smaller, seemingly less fit plants may be capable of sustaining the invasion.

A broad conclusion from these studies is that multiple introductions and high $H_{\mathrm{S}}$ in centrally located source populations facilitates selective processes and increase fitness of individuals in edge populations. By applying management strategies to populations that are feeding the invasion at the range edge, the spread of invasive plants can be reduced. 


\section{References}

Ackerly, D. D. 2003. Community assembly, niche conservatism, and adaptive evolution in changing environments. International Journal of Plant Sciences 164:S165S184.

Aikio, S., R. P. Duncan, and P. E. Hulme. 2010. Lag-phases in alien plant invasions: separating the facts from the artefacts. Oikos 119:370-378.

Alexander, J. M., P. J. Edwards, M. Poll, C. G. Parks, and H. Dietz. 2009. Establishment of parallel altitudinal clines in traits of native and introduced forbs. Ecology 90:612-622.

Arnaud-Haond, S., S. Teixeira, S. I. Massa, C. Billot, P. Saenger, G. Coupland, C. M. Duarte, and E. A. Serrao. 2006. Genetic structure at range edge: low diversity and high inbreeding in Southeast Asian mangrove (Avicennia marina) populations. Mol Ecol 15:3515-3525.

Aroca, R., R. Porcel, and J. Manuel Ruiz-Lozano. 2012. Regulation of root water uptake under abiotic stress conditions. Journal of Experimental Botany 63:43-57.

Barrett, S. C. H. and D. Charlesworth. 1991. Effects of a change in the level of inbreeding on the genetic load. Nature 352:522-524.

Barringer, B. C., E. A. Kulka, and L. F. Galloway. 2012. Reduced inbreeding depression in peripheral relative to central populations of a monocarpic herb. J. Evol. Biol. 25:1200-1208.

Bartlett, M. K., C. Scoffoni, and L. Sack. 2012. The determinants of leaf turgor loss point and prediction of drought tolerance of species and biomes: a global meta-analysis. Ecol. Lett. 15:393-405.

Beerling, D. J. and W. G. Chaloner. 1992. Stomatal density as an indicator of atmospheric $\mathrm{CO} 2$ concentration. Holocene 2:71-78.

Bijlsma, R. and V. Loeschcke. 2012. Genetic erosion impedes adaptive responses to stressful environments. Evol. Appl. 5:117-129.

Blair, A. C. and L. M. Wolfe. 2004. The evolution of an invasive plant: an experimental study with Silene latifolia. Ecology 85:3035-3042.

Bock, D. G., C. Caseys, R. D. Cousens, M. A. Hahn, S. M. Heredia, S. Hübner, K. G. Turner, K. D. Whitney, and L. H. Rieseberg. 2015. What we still don't know about invasion genetics. Molecular ecology 24:2277-2297.

Booth, M. S., M. M. Caldwell, and J. M. Stark. 2003. Overlapping resource use in three Great Basin species: implications for community invasibility and vegetation dynamics. Journal of Ecology 91:36-48.

Bossdorf, O., H. Auge, L. Lafuma, W. E. Rogers, E. Siemann, and D. Prati. 2005. Phenotypic and genetic differentiation between native and introduced plant populations. Oecologia 144:1-11.

Brown, A. H. D. and D. R. Marshall. 1980. Evolutionary changes accompanying colonization in plants. The University of British Columbia. Second International Congress of Systematic and Evolutionary Biology, Vancouver, B.C., Canada, July 17-24, 1980. I+441p. University of British Columbia: Vancouver, B.C., Canada. Paper:P38-P38. 
Brown, J. and A. Idris. 2005. Genetic differentiation of whitefly Bemisia tabaci mitochondrial cytochrome oxidase I, and phylogeographic concordance with the coat protein of the plant virus genus Begomovirus. Annals of the Entomological Society of America 98:827-837.

Burns, J. H. 2004. A comparison of invasive and non-invasive dayflowers (Commelinaceae) across experimental nutrient and water gradients. Diversity and Distributions 10:387-397.

Burns, J. H. and A. A. Winn. 2006. A comparison of plastic responses to competition by invasive and non-invasive congeners in the Commelinaceae. Biological Invasions 8:797-807.

Byers, D. L. and D. M. Waller. 1999. Do plant populations purge their genetic load? Effects of population size and mating history on inbreeding depression. Annu. Rev. Ecol. Syst. 30:479-513.

Callahan, H. S., H. Maughan, and U. K. Steiner. 2008. Phenotypic plasticity, costs of phenotypes, and costs of plasticity. Annals of the New York Academy of Sciences 1133:44-66.

Callaway, R. M. and J. L. Maron. 2006. What have exotic plant invasions taught us over the past 20 years? Trends in Ecology \& Evolution 21:369-374.

Callaway, R. M., S. C. Pennings, and C. L. Richards. 2003. Phenotypic plasticity and interactions among plants. Ecology 84:1115-1128.

Callaway, R. M. and L. R. Walker. 1997. Competition and facilitation: A synthetic approach to interactions in plant communities. Ecology 78:1958-1965.

Campbell, B. D. and J. P. Grime. 1992. An experimental test of plant strategy theory. Ecology 73:15-29.

Carr, D. E. and M. R. Dudash. 2003. Recent approaches into the genetic basis of inbreeding depression in plants. Philosophical Transactions of the Royal Society of London Series B-Biological Sciences 358:1071-1084.

Catalan, P. and R. G. Olmstead. 2000. Phylogenetic reconstruction of the genus Branchypodium P-Beauv. (Poaceae) from combined sequences of chloroplast ndhF gene and nuclear ITS. Plant Systematics and Evolution 220:1-19.

Charlesworth, D. and B. Charlesworth. 1987. Inbreeding Depression and its Evolutionary Consequences. Annual Review of Ecology and Systematics 18:237-268.

Charlesworth, D. and J. H. Willis. 2009. The genetics of inbreeding depression. Nature Reviews Genetics 10:783-796.

Chaves, M. M., J. P. Maroco, and J. S. Pereira. 2003. Understanding plant responses to drought - from genes to the whole plant. Functional Plant Biology 30:239-264.

Cheptou, P. O. and K. Donohue. 2011. Environment-dependent inbreeding depression: its ecological and evolutionary significance. New Phytologist 189:395-407.

Cheptou, P. O., J. Lepart, and J. Escarre. 2001. Inbreeding depression under intraspecific competition in a highly outcrossing population of Crepis sancta (Asteraceae): Evidence for frequency-dependent variation. American Journal of Botany 88:1424-1429.

Colautti, R. I., C. G. Eckert, and S. C. Barrett. 2010. Evolutionary constraints on adaptive evolution during range expansion in an invasive plant. Proceedings of the Royal Society of London B: Biological Sciences 277:1799-1806. 
Crnokrak, P. and S. C. H. Barrett. 2002. Perspective: Purging the genetic load: A review of the experimental evidence. Evolution 56:2347-2358.

Crow, J. F. and M. Kimura. 1970. An introduction to population genetics theory. An introduction to population genetics theory.

Cruzan, M. B., J. L. Hamrick, M. L. Arnold, and B. D. Bennett. 1994. Mating system variation in hybridizing irises: effects of phenology and floral densities on family outcrossing rates. Heredity 72:95-105.

Cutter, A. D. and B. A. Payseur. 2013. Genomic signatures of selection at linked sites: unifying the disparity among species. Nature Reviews Genetics 14:262-274.

Damgaard, C. and V. Loeschcke. 1994. Inbreeding depression and dominance suppression competition after inbreeding in rapeseed (Brassica-napus). Theor Appl Genet 88:321-323.

Darling, J. A., M. J. Bagley, J. Roman, C. K. Tepolt, and J. B. Geller. 2008. Genetic patterns across multiple introductions of the globally invasive crab genus Carcinus. Mol. Ecol. 17:4992-5007.

Davidson, A. M., M. Jennions, and A. B. Nicotra. 2011. Do invasive species show higher phenotypic plasticity than native species and, if so, is it adaptive? A metaanalysis. Ecology letters 14:419-431.

Denver, D. R., K. Morris, M. Lynch, and W. K. Thomas. 2004. High mutation rate and predominance of insertions in the Caenorhabditis elegans nuclear genome. Nature 430:679-682.

DeWalt, S. J. and J. L. Hamrick. 2004. Genetic variation of introduced Hawaiian and native Costa Rican populations of an invasive tropical shrub, Clidemia hirta (Melastomataceae). American Journal of Botany 91:1155-1162.

DeWitt, T. J., A. Sih, and D. S. Wilson. 1998. Costs and limits of phenotypic plasticity. Trends in Ecology \& Evolution 13:77-81.

Dlugosch, K. M. and C. G. Hays. 2008. Genotypes on the move: some things old and some things new shape the genetics of colonization during species invasions. Mol Ecol 17:4583-4585.

Dlugosch, K. M. and I. M. Parker. 2008a. Founding events in species invasions: genetic variation, adaptive evolution, and the role of multiple introductions. Mol Ecol 17:431-449.

Dlugosch, K. M. and I. M. Parker. 2008b. Invading populations of an ornamental shrub show rapid life history evolution despite genetic bottlenecks. Ecology letters 11:701-709.

Donohue, K. 1998. Effects of inbreeding on traits that influence dispersal and progeny density in Cakile edentula var Lacustris (Brassicaceae). Am. J. Bot. 85:661-668.

Droste, T., S. L. Flory, and K. Clay. 2010. Variation for phenotypic plasticity among populations of an invasive exotic grass. Plant Ecology 207:297-306.

Dybdahl, M. F. and S. L. Kane. 2005. Adaptation vs. phenotypic plasticity in the success of a clonal invader. Ecology 86:1592-1601.

Ellstrand, N. C. and K. A. Schierenbeck. 2000. Hybridization as a stimulus for the evolution of invasiveness in plants? Proc. Natl. Acad. Sci. U. S. A. 97:7043-7050. 
Endels, P., H. Jacquemyn, R. Brys, and M. Hermy. 2007. Genetic erosion explains deviation from demographic response to disturbance and year variation in relic populations of the perennial Primula vulgaris. J. Ecol. 95:960-972.

Eriksen, R. L., T. Desronvil, J. L. Hierro, and R. Kesseli. 2012. Morphological differentiation in a common garden experiment among native and non-native specimens of the invasive weed yellow starthistle (Centaurea solstitialis). Biological Invasions 14:1459-1467.

Espeland, E. K. 2013. Predicting the dynamics of local adaptation in invasive species. Journal of Arid Land 5:268-274.

Evans, R. D., R. Rimer, L. Sperry, and J. Belnap. 2001. Exotic plant invasion alters nitrogen dynamics in an arid grassland. Ecological Applications 11:1301-1310.

Excoffier, L. 2004. Patterns of DNA sequence diversity and genetic structure after a range expansion: lessons from the infinite-island model. Mol Ecol 13:853-864.

Excoffier, L., M. Foll, and R. J. Petit. 2009. Genetic Consequences of Range Expansions. Annual Review of Ecology Evolution and Systematics 40:481-501.

Eyre-Walker, A. and P. D. Keightley. 2007. The distribution of fitness effects of new mutations. Nature Reviews Genetics 8:610-618.

Facon, B., J.-P. Pointier, P. Jarne, V. Sarda, and P. David. 2008. High genetic variance in life-history strategies within invasive populations by way of multiple introductions. Curr. Biol. 18:363-367.

Farooq, M., A. Wahid, N. Kobayashi, D. Fujita, and S. Basra. 2009. Plant drought stress: effects, mechanisms and management. Pp. 153-188. Sustainable Agriculture. Springer.

Felker - Quinn, E., J. A. Schweitzer, and J. K. Bailey. 2013. Meta - analysis reveals evolution in invasive plant species but little support for Evolution of Increased Competitive Ability (EICA). Ecology and evolution 3:739-751.

Fenn, M. E., J. S. Baron, E. B. Allen, H. M. Rueth, K. R. Nydick, L. Geiser, W. D. Bowman, J. O. Sickman, T. Meixner, D. W. Johnson, and P. Neitlich. 2003. Ecological effects of nitrogen deposition in the western United States. Bioscience 53:404-420.

Fisher, R. A. 1941. Average excess and average effect of a gene substitution. Annals of Eugenics 11:53-63.

Flores-Moreno, H., E. S. Garcia-Trevino, A. D. Letten, and A. T. Moles. 2015. In the beginning: phenotypic change in three invasive species through their first two centuries since introduction. Biological Invasions 17:1215-1225.

Fox, C. W. and D. H. Reed. 2010. Inbreeding depression increases with maternal age in a seed-feeding beetle. Evolutionary Ecology Research 12:961-972.

Fox, C. W. and D. H. Reed. 2011. Inbreeding depression increases with environmental stress: an experimental study and meta-analysis. Evolution 65:246-258.

Frankham, R. 1997. Do island populations have less genetic variation than mainland populations? Heredity 78:311-327.

Frankham, R. 2004. Resolving the genetic paradox in invasive species. Heredity 94:385385 .

Franks, S. J. and J. Munshi-South. 2014. Go forth, evolve and prosper: the genetic basis of adaptive evolution in an invasive species. Mol. Ecol. 23:2137-2140. 
Galloway, J. N., A. R. Townsend, J. W. Erisman, M. Bekunda, Z. C. Cai, J. R. Freney, L. A. Martinelli, S. P. Seitzinger, and M. A. Sutton. 2008. Transformation of the nitrogen cycle: Recent trends, questions, and potential solutions. Science 320:889892.

GarciaRamos, G. and M. Kirkpatrick. 1997. Genetic models of adaptation and gene flow in peripheral populations. Evolution 51:21-28.

Garza, J. C. and E. G. Williamson. 2001. Detection of reduction in population size using data from microsatellite loci. Mol Ecol 10:305-318.

Gaskin, J. F. and B. A. Schaal. 2002. Hybrid Tamarix widespread in US invasion and undetected in native Asian range. Proceedings of the National Academy of Sciences 99:11256-11259.

Geng, Y.-P., X.-Y. Pan, C.-Y. Xu, W.-J. Zhang, B. Li, J.-K. Chen, B.-R. Lu, and Z.-P. Song. 2007. Phenotypic plasticity rather than locally adapted ecotypes allows the invasive alligator weed to colonize a wide range of habitats. Biological Invasions 9:245-256.

Gerry, A. K. and S. D. Wilson. 1995. The influence of initial size on the competitive responses of 6 plant-species. Ecology 76:272-279.

Ghalambor, C. K., J. K. McKay, S. P. Carroll, and D. N. Reznick. 2007. Adaptive versus non - adaptive phenotypic plasticity and the potential for contemporary adaptation in new environments. Functional Ecology 21:394-407.

Gilbert, B., R. Turkington, and D. S. Srivastava. 2009. Dominant Species and Diversity: Linking Relative Abundance to Controls of Species Establishment. Am Nat 174:850-862.

Godoy, O., F. Valladares, and P. Castro - Díez. 2011. Multispecies comparison reveals that invasive and native plants differ in their traits but not in their plasticity. Functional Ecology 25:1248-1259.

Goodwillie, C. and M. C. Knight. 2006. Inbreeding depression and mixed mating in Leptosiphon jepsonii: A comparison of three populations. Ann. Bot. 98:351-360.

Goudet, J. 1995. FSTAT (Version 1.2): A Computer Program to Calculate F-Statistics. Journal of Heredity 86:485-486.

Goudet, J. 2005. HIERFSTAT, a package for R to compute and test hierarchical Fstatistics. Molecular Ecology Notes 5:184-186.

Green, E. K. and S. M. Galatowitsch. 2002. Effects of Phalaris arundinacea and nitrate-N addition on the establishment of wetland plant communities. Journal of Applied Ecology 39:134-144.

Grime, J. 1977. Evidence for the existence of three primary strategies in plants and its relevance to ecological and evolutionary theory. American naturalist:1169-1194.

Grime, J. P. 2001. Plant functional types, communities and ecosystems. Ecology: Achievement and Challenge:161-181.

Grotkopp, E. and M. Rejmánek. 2007. High seedling relative growth rate and specific leaf area are traits of invasive species: phylogenetically independent contrasts of woody angiosperms. American Journal of Botany 94:526-532.

Haag-Liautard, C., M. Dorris, X. Maside, S. Macaskill, D. L. Halligan, B. Charlesworth, and P. D. Keightley. 2007. Direct estimation of per nucleotide and genomic deleterious mutation rates in Drosophila. Nature 445:82-85. 
Hacke, U. G. and J. S. Sperry. 2001. Functional and ecological xylem anatomy. Perspectives in plant ecology, evolution and systematics 4:97-115.

Hahn, M. A., M. van Kleunen, and H. Müller-Schärer. 2012. Increased phenotypic plasticity to climate may have boosted the invasion success of polyploid Centaurea stoebe.

Haikola, S., W. Fortelius, R. B. O'Hara, M. Kuussaari, N. Wahlberg, I. J. Saccheri, M. C. Singer, and I. Hanski. 2001. Inbreeding depression and the maintenance of genetic load in Melitaea cinxia metapopulations. Conserv. Genet. 2:325-335.

Halligan, D. L. and P. D. Keightley. 2009. Spontaneous Mutation Accumulation Studies in Evolutionary Genetics. Pp. 151-172. Annual Review of Ecology Evolution and Systematics.

Hartl, D. L. and A. G. Clark. 2006. Principles of Population Genetics. Sinauer Associates, Inc., Sunderland, Massachsetts.

Hayes, C. N., J. A. Winsor, and A. G. Stephenson. 2005. Environmental variation influences the magnitude of inbreeding depression in Cucurbita pepo ssp texana (Cucurbitaceae). J. Evol. Biol. 18:147-155.

Hayes, K. R. and S. C. Barry. 2008. Are there any consistent predictors of invasion success? Biological Invasions 10:483-506.

Hearn, P. P., T. Hare, P. Shruben, D. Sherrill, C. LaMar, and P. Tsushima. 2003. Global GIS: Global Coverage. American Geological Institute. Geological Survey (U.S.).

Hedrick, P. W. and R. Fredrickson. 2010. Genetic rescue guidelines with examples from Mexican wolves and Florida panthers. Conserv. Genet. 11:615-626.

Hedrick, P. W. and S. T. Kalinowski. 2000. Inbreeding depression in conservation biology. Annu. Rev. Ecol. Syst. 31:139-162.

Hijmans, R. J., S. E. Cameron, J. L. Parra, P. G. Jones, and A. Jarvis. 2005. Very high resolution interpolated climate surfaces for global land areas. International Journal of Climatology 25:1965-1978.

Hodgins, K. and L. Rieseberg. 2011. Genetic differentiation in life - history traits of introduced and native common ragweed (Ambrosia artemisiifolia) populations. Journal of evolutionary biology 24:2731-2749.

Holdredge, C., M. D. Bertness, E. von Wettberg, and B. R. Silliman. 2010. Nutrient enrichment enhances hidden differences in phenotype to drive a cryptic plant invasion. Oikos 119:1776-1784.

Holmes, S. E., B. A. Roy, J. P. Reed, and B. R. Johnson. 2010. Context-dependent pattern and process: the distribution and competitive dynamics of an invasive grass, Brachypodium sylvaticum. Biol Invasions 12:2303-2318.

Hull, A. C., Jr. 1974. Species for Seeding Mountain Rangelands in Southeastern Idaho, Northeastern Utah, and Western Wyoming. Journal of Range Management 27:150-153.

Hulme, P. E. 2009. Trade, transport and trouble: managing invasive species pathways in an era of globalization. Journal of Applied Ecology 46:10-18.

Ibanez, I., J. S. Clark, and M. C. Dietze. 2009. Estimating colonization potential of migrant tree species. Global Change Biology 15:1173-1188. 
Ibrahim, K. M., R. A. Nichols, and G. M. Hewitt. 1995. Spatial patterns of genetic variation generated by different forms of dispersal during range expansion. Heredity 77:282-291.

Jarne, P. and D. Charlesworth. 1993. The Evolution of the Selfing Rate in Functionally Hermaphrodite Plants and Animals. Annu. Rev. Ecol. Syst. 24:441-466.

Johnson, W. E., D. P. Onorato, M. E. Roelke, E. D. Land, M. Cunningham, R. C. Belden, R. McBride, D. Jansen, M. Lotz, D. Shindle, J. Howard, D. E. Wildt, L. M. Penfold, J. A. Hostetler, M. K. Oli, and S. J. O'Brien. 2010. Genetic Restoration of the Florida Panther. Science 329:1641-1645.

Kadioglu, A., R. Terzi, N. Saruhan, and A. Saglam. 2012. Current advances in the investigation of leaf rolling caused by biotic and abiotic stress factors. Plant Science 182:42-48.

Kaye, T. 2003. Invasive Plant Alert: False-Brome (Brachypodium sylvaticum). False Brome Working Group. Institute for Applied Ecology. Corvallis, Oregon.

Keller, L. F. and D. M. Waller. 2002. Inbreeding effects in wild populations. Trends Ecol. Evol. 17:230-241.

Keller, S. R. and D. R. Taylor. 2008. History, chance and adaptation during biological invasion: separating stochastic phenotypic evolution from response to selection. Ecol. Lett. 11:852-866.

Kilkenny, F. F. and L. F. Galloway. 2013. Adaptive divergence at the margin of an invaded range. Evolution 67:722-731.

Knops, J. M. H. and K. Reinhart. 2000. Specific leaf area along a nitrogen fertilization gradient. American Midland Naturalist 144:265-272.

Kolbe, J. J., P. S. VanMiddlesworth, N. Losin, N. Dappen, and J. B. Losos. 2012. Climatic niche shift predicts thermal trait response in one but not both introductions of the Puerto Rican lizard Anolis cristatellus to Miami, Florida, USA. Ecology and evolution 2:1503-1516.

Lamb, E. G. and J. F. Cahill. 2008. When competition does not matter: Grassland diversity and community composition. Am Nat 171:777-787.

Lambrinos, J. G. 2004. How interactions between ecology and evolution influence contemporary invasion dynamics. Ecology 85:2061-2070.

Lamont, B. B. and H. C. Lamont. 2000. Utilizable water in leaves of 8 arid species as derived from pressure-volume curves and chlorophyll fluorescence. Physiologia Plantarum 110:64-71.

Lande, R. 1992. Neutral theory of quantitative genetic variance in an island model with local extinction and colonization. Evolution 46:381-389.

Lande, R. 2009. Adaptation to an extraordinary environment by evolution of phenotypic plasticity and genetic assimilation. Journal of evolutionary biology 22:1435-1446.

Lande, R. 2015. Evolution of phenotypic plasticity in colonizing species. Molecular ecology 24:2038-2045.

Lande, R. and S. J. Arnold. 1983. The Measurement of Selection on Correlated Characters. Evolution 37:1210-1226.

Lande, R. and D. Schemske. 1985. The evolution of self-fertilization and inbreeding depression in plants. I. Genetic models. Evolution 39:24-40. 
Lande, R., D. W. Schemske, and S. T. Schultz. 1994. High inbreeding depression, selective interference among loci, and the threshold selfing rate for purging recessive lethal mutations. Evolution 48:965-978.

Larcher, W. 1995. Physiological plant ecology: Ecophysiology and stress physiology of functional groups, Third edition.

Lavergne, S. and J. Molofsky. 2007. Increased genetic variation and evolutionary potential drive the success of an invasive grass. Proceedings of the National Academy of Sciences of the United States of America 104:3883-3888.

Leberg, P. L. and B. D. Firmin. 2008. Role of inbreeding depression and purging in captive breeding and restoration programmes. Mol. Ecol. 17:334-343.

Lee, C. E. 2002. Evolutionary genetics of invasive species. Trends in Ecology \& Evolution 17:386-391.

Leinonen, T., R. J. S. McCairns, R. B. O'Hara, and J. Merila. 2013. Q(ST)-F-ST comparisons: evolutionary and ecological insights from genomic heterogeneity. Nat. Rev. Genet. 14:179-190.

Leishman, M. R., T. Haslehurst, A. Ares, and Z. Baruch. 2007. Leaf trait relationships of native and invasive plants: community- and global-scale comparisons. New Phytologist 176:635-643.

Lenda, M., P. Skorka, J. M. H. Knops, D. Moron, S. Tworek, and M. Woyciechowski. 2012. Plant establishment and invasions: an increase in a seed disperser combined with land abandonment causes an invasion of the non-native walnut in Europe. Proceedings of the Royal Society B-Biological Sciences 279:1491-1497.

Liancourt, P. and K. Tielbörger. 2009. Competition and a short growing season lead to ecotypic differentiation at the two extremes of the ecological range. Functional Ecology 23:397-404.

Lynch, M., J. Blanchard, D. Houle, T. Kibota, S. Schultz, L. Vassilieva, and J. Willis. 1999. Perspective: Spontaneous deleterious mutation. Evolution 53:645-663.

Mack, R. N., D. Simberloff, W. M. Lonsdale, H. Evans, M. Clout, and F. A. Bazzaz. 2000. Biotic invasions: Causes, epidemiology, global consequences, and control. Ecological Applications 10:689-710.

Malmstrom, V. H. 1969. New approach to classification of climate. Journal of Geography 68:351-357.

Marchini, G.L., N.C. Sherlock, A.P. Ramakrishnan, D.M. Rosenthal, and M.B. Cruzan. In press. Rapid Purging of Genetic Load in a Metapopulation and Consequences for Range Expansion in an Invasive Plant. Biological Invasions. DOI: 10.1007/s10530-015-1001-5

Maron, J. L., M. Vila, R. Bommarco, S. Elmendorf, and P. Beardsley. 2004. Rapid evolution of an invasive plant. Ecological Monographs 74:261-280.

Martina, J. P. and C. N. von Ende. 2012. Highly plastic response in morphological and physiological traits to light, soil-N and moisture in the model invasive plant, Phalaris arundinacea. Environmental and Experimental Botany 82:43-53.

Matesanz, S., T. Horgan-Kobelski, and S. E. Sultan. 2012. Phenotypic Plasticity and Population Differentiation in an Ongoing Species Invasion. Plos One 7:13.

Matzek, V. 2012. Trait values, not trait plasticity, best explain invasive species' performance in a changing environment. 
Meier, I. C. and C. Leuschner. 2008. Leaf size and leaf area index in Fagus sylvatica forests: competing effects of precipitation, temperature, and nitrogen availability. Ecosystems 11:655-669.

Meimberg, H., N. F. Milan, M. Karatassiou, E. K. Espeland, J. K. McKay, and K. J. Rice. 2010. Patterns of introduction and adaptation during the invasion of Aegilops triuncialis (Poaceae) into Californian serpentine soils. Mol. Ecol. 19:5308-5319.

Meinzer, F. C. and D. A. Grantz. 1990. Stomatal and hydraulic conductance in growing sugarcane - stomatal adjustment to water transport capacity. Plant Cell and Environment 13:383-388.

Miller, B. M., R. J. Aitken, M. J. Oldham, and A. A. Reznicek. 2011. Slender False Brome (Brachypodium sylvaticum, Poaceae), an Invasive Grass New to Ontario, Canada. Can Field Nat 125:235-240.

Mooney, H. A. and E. E. Cleland. 2001. The evolutionary impact of invasive species. Proceedings of the National Academy of Sciences of the United States of America 98:5446-5451.

Mooney, H. A. and R. J. Hobbs. 2000. Invasive Species in a Changing World. Island Press, Washington, D.C.

Morgante, M. and A. M. Olivieri. 1993. Pcr- amplified microsatellites as markers in plant genetics. Plant J 3:175-182.

Moser, B., J. D. Fridley, A. P. Askew, and J. P. Grime. 2011. Simulated migration in a long-term climate change experiment: invasions impeded by dispersal limitation, not biotic resistance. J Ecol 99:1229-1236.

Muller-Scharer, H., U. Schaffner, and T. Steinger. 2004. Evolution in invasive plants: implications for biological control. Trends in Ecology \& Evolution 19:417-422.

Nagylaki, T. 1976. A model for the evolution of self fertilization and vegetative reproduction. J. Theor. Biol. 58:55-58.

Neher, R. A. 2013. Genetic Draft, Selective Interference, and Population Genetics of Rapid Adaptation. Annual Review of Ecology, Evolution, and Systematics 44:195-215.

Niinemets, Ü., F. Valladares, and R. Ceulemans. 2003. Leaf - level phenotypic variability and plasticity of invasive Rhododendron ponticum and non - invasive Ilex aquifolium co - occurring at two contrasting European sites. Plant, Cell \& Environment 26:941-956.

Novak, S. J. and R. N. Mack. 1995. Allozyme diversity in the apomictic vine Bryoniaalba (Cucurbitaceae) - Potential consequences of multiple introductions. American Journal of Botany 82:1153-1162.

O'Toole, J. C. and R. T. Cruz. 1980. Response of leaf water potential, stomatal resistance, and leaf rolling to water stress. Plant physiology 65:428-432.

Olivieri, I. 2009. Alternative mechanisms of range expansion are associated with different changes of evolutionary potential. Trends in Ecology \& Evolution 24:289-292.

Ossowski, S., K. Schneeberger, J. I. Lucas-Lledo, N. Warthmann, R. M. Clark, R. G. Shaw, D. Weigel, and M. Lynch. 2010. The Rate and Molecular Spectrum of Spontaneous Mutations in Arabidopsis thaliana. Science 327:92-94. 
Palacio - López, K. and E. Gianoli. 2011. Invasive plants do not display greater phenotypic plasticity than their native or non - invasive counterparts: a meta analysis. Oikos 120:1393-1401.

Pattison, R., G. Goldstein, and A. Ares. 1998. Growth, biomass allocation and photosynthesis of invasive and native Hawaiian rainforest species. Oecologia 117:449-459.

Pearson, G. A., A. Lago-Leston, and C. Mota. 2009. Frayed at the edges: selective pressure and adaptive response to abiotic stressors are mismatched in low diversity edge populations. J Ecol 97:450-462.

Petrů, M., K. Tielbörger, R. Belkin, M. Sternberg, and F. Jeltsch. 2006. Life history variation in an annual plant under two opposing environmental constraints along an aridity gradient. Ecography 29:66-74.

Picotte, J., D. Rosenthal, J. Rhode, and M. Cruzan. 2007. Plastic responses to temporal variation in moisture availability: consequences for water use efficiency and plant performance. Oecologia 153:821-832.

Piersma, T. and J. Drent. 2003. Phenotypic flexibility and the evolution of organismal design. Trends in Ecology \& Evolution 18:228-233.

Pigliucci, M. and C. J. Murren. 2003. Perspective: Genetic assimilation and a possible evolutionary paradox: can macroevolution sometimes be so fast as to pass us by? Evolution 57:1455-1464.

Pimentel, D., R. Zuniga, and D. Morrison. 2005. Update on the environmental and economic costs associated with alien-invasive species in the United States. Ecological Economics 52:273-288.

Pinheiro J, B. D., DebRoy S, Sarkar D and R Core Team 2015._nlme: Linear and Nonlinear Mixed Effects Models_. R package version 3.1-120.

Piry, S., A. Alapetite, J. M. Cornuet, D. Paetkau, L. Baudouin, and A. Estoup. 2004. GENECLASS2: A software for genetic assignment and first-generation migrant detection. Journal of Heredity 95:536-539.

Poorter, H., U. Niinemets, L. Poorter, I. J. Wright, and R. Villar. 2009. Causes and consequences of variation in leaf mass per area (LMA): a meta-analysis. New Phytologist 182:565-588.

Prentis, P. J., J. R. U. Wilson, E. E. Dormontt, D. M. Richardson, and A. J. Lowe. 2008. Adaptive evolution in invasive species. Trends in Plant Science 13:288-294.

Pujol, B., S.-R. Zhou, J. S. Vilas, and J. R. Pannell. 2009. Reduced inbreeding depression after species range expansion. Proceedings of the National Academy of Sciences of the United States of America 106:15379-15383.

R Core Development Team. 2014. R: A language and environment for statistical computing. R Foundation for Statistical Computing, Vienna, Austria.

Ramakrishnan, A. P., T. Musial, and M. B. Cruzan. 2010. Shifting dispersal modes at an expanding species' range margin. Mol Ecol 19:1134-1146.

Ramakrishnan, A. P., D. M. Rosenthal, T. Musial, and M. B. Cruzan. 2008. Isolation and characterization of nine microsatellite markers for Brachypodium sylvaticum (Huds.) Beauv., a recently invasive grass species in Oregon. Molecular Ecology Resources 8:1297-1299. 
Rannala, B. and J. L. Mountain. 1997. Detecting immigration by using multilocus genotypes. Proceedings of the National Academy of Sciences of the United States of America 94:9197-9201.

Richards, C. L., O. Bossdorf, N. Z. Muth, J. Gurevitch, and M. Pigliucci. 2006. Jack of all trades, master of some? On the role of phenotypic plasticity in plant invasions. Ecology letters 9:981-993.

Richards, C. M. 2000. Inbreeding depression and genetic rescue in a plant metapopulation. Am. Nat. 155:383-394.

Richardson, D. M. and M. Rejmánek. 2011. Trees and shrubs as invasive alien species a global review. Diversity and Distributions 17:788-809.

Rieseberg, L. H., H. Choi, R. Chan, and C. Spore. 1993. Genomic map of a diploid hybrid species. Heredity 70:285-293.

Riis, T., C. Lambertini, B. Olesen, J. S. Clayton, H. Brix, and B. K. Sorrell. 2010. Invasion strategies in clonal aquatic plants: are phenotypic differences caused by phenotypic plasticity or local adaptation? Annals of botany:mcq176.

Roman, J. and J. A. Darling. 2007. Paradox lost: genetic diversity and the success of aquatic invasions. Trends Ecol. Evol. 22:454-464.

Rosenthal, D. M., A. P. Ramakrishnan, and M. B. Cruzan. 2008a. Evidence for multiple sources and intraspecific hybridization at early stages of the invasion of

Brachypodium sylvaticum (Hudson) Beauv. in North America. Molecular Ecology 17:4657-4669.

Rosenthal, D. M., A. P. Ramakrishnan, and M. B. Cruzan. 2008b. Evidence for multiple sources of invasion and intraspecific hybridization in Brachypodium sylvaticum (Hudson) Beauv. in North America. Mol Ecol 17:4657-4669.

Roy, B. A. 2010. Brachypodium sylvaticum. Invasive Species Compendium. CAB International Publishing, Wallingford, UK.

Roy, B. A., T. Coulson, W. Blaser, T. Policha, J. L. Stewart, G. K. Blaisdell, and S. Guesewell. 2011. Population regulation by enemies of the grass Brachypodium sylvaticum: demography in native and invaded ranges. Ecology 92:665-675.

Royer, D. L. 2001. Stomatal density and stomatal index as indicators of paleoatmospheric CO2 concentration. Review of Palaeobotany and Palynology 114:1-28.

Sack, L., P. D. Cowan, N. Jaikumar, and N. M. Holbrook. 2003. The 'hydrology' of leaves: co-ordination of structure and function in temperate woody species. Plant Cell and Environment 26:1343-1356.

Sack, L., M. T. Tyree, and N. M. Holbrook. 2005. Leaf hydraulic architecture correlates with regeneration irradiance in tropical rainforest trees. New Phytologist 167:403413.

Sakai, A. K., F. W. Allendorf, J. S. Holt, D. M. Lodge, J. Molofsky, K. A. With, S. Baughman, R. J. Cabin, J. E. Cohen, N. C. Ellstrand, D. E. McCauley, P. O'Neil, I. M. Parker, J. N. Thompson, and S. G. Weller. 2001. The population biology of invasive species. Annual Review of Ecology and Systematics 32:305-332.

Sambatti, J. and K. J. Rice. 2007. Functional ecology of ecotypic differentiation in the Californian serpentine sunflower (Helianthus exilis). New Phytologist 175:107119. 
Schoch, P. G., C. Zinsou, and M. Sibi. 1980. Dependence of the stomatal index on environmental-factors during stomatal differentiation in leaves of Vigna-sinensis L. 1. Effect of light-intensity. Journal of Experimental Botany 31:1211-1216.

Selkoe, K. A. and R. J. Toonen. 2006. Microsatellites for ecologists: a practical guide to using and evaluating microsatellite markers. Ecol. Lett. 9:615-629.

Sexton, J. P., J. K. McKay, and A. Sala. 2002. Plasticity and genetic diversity may allow saltcedar to invade cold climates in North America. Ecological Applications 12:1652-1660.

Shields, W. M. 1993. The natural and unnatural history of inbreeding and outbreeding. The natural history of inbreeding and outbreeding:143-169.

Siemann, E. and W. E. Rogers. 2003. Increased competitive ability of an invasive tree may be limited by an invasive beetle. Ecological Applications 13:1503-1507.

Smith, M. S., J. D. Fridley, J. Yin, and T. L. Bauerle. 2013. < bold $>$ Contrasting xylem vessel constraints on hydraulic conductivity between native and non-native woody understory species $</$ bold $>$. Frontiers in Plant Science 4.

Sperry, J. S. and N. Z. Saliendra. 1994. Intra-plant and inter-plant variation in xylem cavitation in Betula occidentalis. Plant Cell and Environment 17:1233-1241.

Spitze, K. 1993. Population-structure in Daphnia-obtusa - Quantitative genetic and allozymic variation. Genetics 135:367-374.

Stinchcombe, J. R., A. F. Agrawal, P. A. Hohenlohe, S. J. Arnold, and M. W. Blows. 2008. Estimating nonlinear selection gradients using quadratic regression coefficients: double or nothing? Evolution 62:2435-2440.

Sultan, S. E., T. Horgan-Kobelski, L. M. Nichols, C. E. Riggs, and R. K. Waples. 2013. A resurrection study reveals rapid adaptive evolution within populations of an invasive plant. Evolutionary Applications 6:266-278.

Swindell, W. R. and J. L. Bouzat. 2006. Selection and inbreeding depression: Effects of inbreeding rate and inbreeding environment. Evolution 60:1014-1022.

Taylor, L. A. V. and M. B. Cruzan. 2015. Propagule Pressure and Disturbance Drive the Invasion of Perennial False-Brome (Brachypodium sylvaticum). Invasive Plant Science and Management 8:169-180.

Thébaud, C. and D. Simberloff. 2001. Are plants really larger in their introduced ranges? The American Naturalist 157:231-236.

Tombesi, S., R. S. Johnson, K. R. Day, and T. M. DeJong. 2010. Relationships between xylem vessel characteristics, calculated axial hydraulic conductance and sizecontrolling capacity of peach rootstocks. Ann. Bot. 105:327-331.

Tsutsui, N. D., A. V. Suarez, D. A. Holway, and T. J. Case. 2000. Reduced genetic variation and the success of an invasive species. Proc. Natl. Acad. Sci. U. S. A. 97:5948-5953.

Turesson, G. 1922. The genotypical response of the plant species to the habitat. Hereditas 3:211-350.

Turner, K. G., H. Fréville, and L. H. Rieseberg. 2015. Adaptive plasticity and niche expansion in an invasive thistle. Ecology and evolution 5:3183-3197.

Turner, N. 1986. Adaptation to water deficits: a changing perspective. Functional Plant Biology 13:175-190. 
Turner, N. C. 1981. Techniques and experimental approaches for the measurement of plant water status. Plant and Soil 58:339-366.

Tyree, M. T. and H. T. Hammel. 1972. The measurement of the turgor pressure and the water relations of plants by the pressure bomb technique. Journal of Experimental Botany 23:267-282.

Tyree, M. T. and M. H. Zimmermann. 2002. Xylem structure and the ascent of sap. Pp. ixiv, 1-283. Xylem structure and the ascent of sap.

Van Kleunen, M. and M. Fischer. 2001. Adaptive evolution of plastic foraging responses in a clonal plant. Ecology 82:3309-3319.

Van Kleunen, M., E. Weber, and M. Fischer. 2010. A meta - analysis of trait differences between invasive and non - invasive plant species. Ecology letters 13:235-245.

van Klinken, R. D., F. D. Panetta, S. Coutts, and B. K. Simon. 2015. Learning from the past to predict the future: an historical analysis of grass invasions in northern Australia. Biological Invasions 17:565-579.

Vellend, M. 2006. The consequences of genetic diversity in competitive communities. Ecology 87:304-311.

Verslues, P. E. and J. K. Zhu. 2005. Before and beyond ABA: upstream sensing and internal signals that determine $\mathrm{ABA}$ accumulation and response under abiotic stress. Biochemical Society Transactions 33:375-379.

Via, S. and R. Lande. 1985. Genotype-Environment Interaction and the Evolution of Phenotypic Plasticity. Evolution 39:505-522.

Vitousek, P. M., J. D. Aber, R. W. Howarth, G. E. Likens, P. A. Matson, D. W. Schindler, W. H. Schlesinger, and D. Tilman. 1997. Human alteration of the global nitrogen cycle: Sources and consequences. Ecological Applications 7:737750.

Walther, G. R. 2010. Community and ecosystem responses to recent climate change. Philos. Trans. R. Soc. B-Biol. Sci. 365:2019-2024.

Weigelt, A. and P. Jolliffe. 2003. Indices of plant competition. Journal of Ecology 91:707-720.

Weis, A. E. and W. L. Gorman. 1990. Measuring selection on reaction norms: an exploration of the Eurosta-Solidago system. Evolution:820-831.

Whitlock, M. C. 2002. Selection, load and inbreeding depression in a large metapopullation. Genetics 160:1191-1202.

Whitlock, M. C. 2008. Evolutionary inference from Q(ST). Mol. Ecol. 17:1885-1896.

Whitlock, M. C. and D. Bourguet. 2000. FACTORS AFFECTING THE GENETIC LOAD IN DROSOPHILA: SYNERGISTIC EPISTASIS AND CORRELATIONS AMONG FITNESS COMPONENTS. Evolution 54:16541660.

Whitlock, M. C. and F. Guillaume. 2009. Testing for Spatially Divergent Selection: Comparing Q(ST) to F-ST. Genetics 183:1055-1063.

Whitney, K. D. and C. A. Gabler. 2008. Rapid evolution in introduced species, 'invasive traits' and recipient communities: challenges for predicting invasive potential. Diversity and Distributions 14:569-580.

Williams, A. C. and B. C. McCarthy. 2001. A new index of interspecific competition for replacement and additive designs. Ecological Research 16:29-40. 
Williams, G. C. 1966. Adaptation and Natural Selection: A Critique of Some Current Evolutionary Thought: A Critique of Some Current Evolutionary Thought. Princeton University Press, Princeton, N.J.

Willis, A., J. Memmott, and R. Forrester. 2000a. Is there evidence for the post - invasion evolution of increased size among invasive plant species? Ecology letters 3:275283.

Willis, A. J., J. Memmott, and R. I. Forrester. 2000b. Is there evidence for the postinvasion evolution of increased size among invasive plant species? Ecol. Lett. 3:275-283.

Wilson, E. E., L. M. Mullen, and D. A. Holway. 2009. Life history plasticity magnifies the ecological effects of a social wasp invasion. Proceedings of the National Academy of Sciences of the United States of America 106:12809-12813.

Winn, A. A., E. Elle, S. Kalisz, P.-O. Cheptou, C. G. Eckert, C. Goodwillie, M. O. Johnston, D. A. Moeller, R. H. Ree, R. D. Sargent, and M. Vallejo-Marin. 2011. Analysis of inbreeding depression in mixed-mating plants provides evidence for selective interference and stable mixed mating. Evolution 65:3339-3359.

Witkowski, E. T. F. 1991. Effects of invasive alien acacias on nutrient cycling in the coastal lowlands of the cape fynbos. Journal of Applied Ecology 28:1-15.

Wright, S. 1931. Evolution in Mendelian Populations. Genetics 16:97-159.

Wright, S. 1977. Evolution and the genetics of populations: experimental results and evolutionary deductions. Vol. 3. Chicago, IL: University of Chicago Press.

$\mathrm{Xu}, \mathrm{Z}$. and G. Zhou. 2008. Responses of leaf stomatal density to water status and its relationship with photosynthesis in a grass. Journal of Experimental Botany 59:3317-3325.

Young, A., T. Boyle, and T. Brown. 1996. The population genetic consequences of habitat fragmentation for plants. Trends in Ecology \& Evolution 11:413-418.

Zalapa, J. E., J. Brunet, and R. P. Guries. 2010. ORIGINAL ARTICLE: The extent of hybridization and its impact on the genetic diversity and population structure of an invasive tree, Ulmus pumila (Ulmaceae). Evolutionary Applications 3:157168.

Zenni, R. D., J.-B. Lamy, L. J. Lamarque, and A. J. Porté. 2014. Adaptive evolution and phenotypic plasticity during naturalization and spread of invasive species: implications for tree invasion biology. Biological Invasions 16:635-644.

Zhao, Y. J., X. J. Yang, X. Q. Xi, X. M. Gao, and S. C. Sun. 2012. Phenotypic Plasticity in the Invasion of Crofton Weed (Eupatorium adenophorum) in China. Weed Sci. 60:431-439.

Zou, J., W. E. Rogers, and E. Siemann. 2009. Plasticity of Sapium sebiferum seedling growth to light and water resources: Inter- and intraspecific comparisons. Basic and Applied Ecology 10:79-88. 


\section{Appendix A}

\section{Supplemental Materials for Chapter 2: Rapid Purging of Genetic Load in a}

\section{Metapopulation and Consequences for Range Expansion in an Invasive Plant}

Figure A.A1 Description of simulations.

Simulations were conducted to mimic selfing lineages within a population (first simulation) or a group of inbred populations with periodic gene flow. Each generation each lineage produced $n$ progeny by selfing or outcrossing to a random individual within the same population. The most fit progeny was chosen to replace the lineage the next generation. If the fitness fell below the survival threshold (defined below) then the lineage was removed. Extinct lineages could be replaced if any lineages in the population surpassed the repopulation threshold (defined below). Propagules from each population were generated to colonize adjacent empty sites if the fitness of any lineage exceeded the colonization fitness threshold (defined below).

If more than one lineage exceeded the

colonization or repopulation threshold then only the one most-fit lineage per population was used for colonization or repopulation.

The simulations were started with $p=$ 0.5 such that the AA, Aa, and aa loci occurred at frequencies of $0.25,0.50$, and 0.25 , respectively.

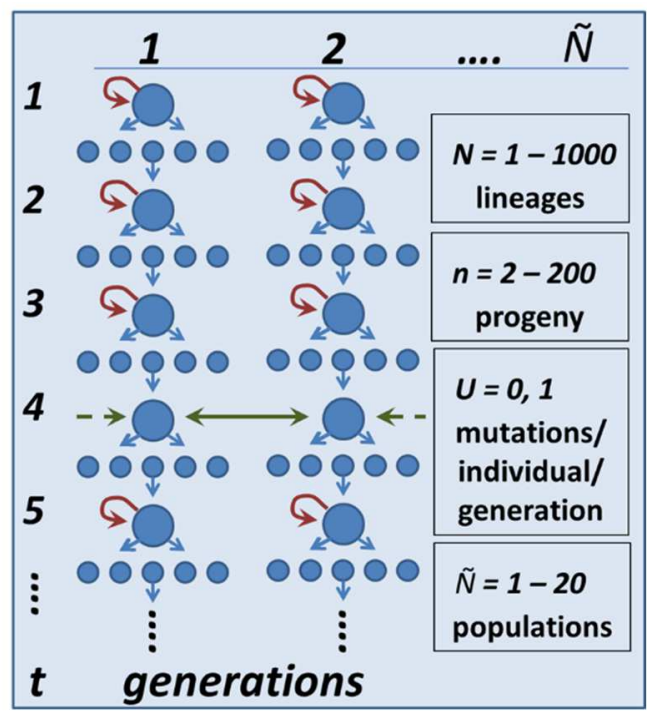

A single individual was generated as the initial colonist of each of the $\widetilde{N}$ populations. At generation 0 this individual was selfed to generate the $N$ individuals to populate each site 
(population). After generation zero each of the $N$ individuals in each population was selfed or outcrossed to generate progeny as described below.

For all simulations reported in this manuscript the deleterious mutations were completely recessive. Fitness for each lineage was calculated as the total number of loci (100 in these simulations) less the number of loci that were homozygous for the deleterious allele (aa). Loci were unlinked and each had an equal effect on fitness for the simulations reported in this manuscript. Mutations were introduced by changing one of the AA loci in each lineage to Aa (introduction of a new deleterious allele).

Each generation gametes were generated by randomly choosing one allele from each locus. Randomly-generated gametes from the same individual (selfing) or a different individual from the same population (outcrossing) were combined to produce $n$ progeny. The most fit progeny was chosen to continue each lineage. Gene flow among populations was accomplished by randomly choosing a single individual to that combined its gametes with a high-fitness individual from a different population.

For the first simulations only one population was used. Lineages within the population were allowed to self and periodically outcross with other individuals from the same population. The effects of the number of progeny and loci on fitness were examined. In these simulations survival thresholds were set at zero so that all selfing lineages survived regardless of their fitness.

For the second simulation $\widetilde{N}=10$ populations consisting of $N=10$ outcrossing lineages were connected by periodic gene flow every five generations. For comparison we used the same set ten of populations with no gene flow with either complete outcrossing or complete selfing, and a single large population of 100 lineages that was isolated and did 
not experience gene flow. Inbreeding within populations led to increased homozygosity, but different populations tended to become fixed for different sets of alleles.

In the first scenario gene flow occurred between randomly-chosen pairs of populations. In this simulation there was no spatial structure so every population had an equal chance of exchanging alleles with every other population. A total of 100 loci were monitored one mutation was allowed to occur in each genome each generation. In the third simulation conditions were exactly the same as the populations of outbreeding individuals connected by gene flow, but we introduced spatial structure and the opportunity for colonization of unoccupied sites to mimic range expansion. Each simulation was established by colonization of five sites by one seed followed by selfing (as described above) at the center of the range. Once populations exceeded the repopulation threshold then randomly-chosen individuals that exceeded the threshold were chosen to contribute to repopulation in populations that had less than ten individuals. Colonization occurred every second generation for sites that were adjacent to a population where the average fitness of lineages exceeded the colonization threshold. Colonization proceeded from propagules produced by randomly-chosen individuals that exceeded the repopulation threshold. Once a population was colonized it could grow to a population size of ten by production of progeny from within the population (if the fitness was greater than the repopulation threshold) or from an adjacent population (if the average fitness exceeded the colonization threshold). We explored the effect of relatedness among populations (equivalent to the initial level of genetic diversity) by varying the number of loci out of 100 that were identical among individuals and 
populations. Genetic diversity of the colonizing populations was estimated using F FT $_{\text {as }}$ described in the manuscript. 
Figure A. A2 To estimate the strength of selection, we compared the maximum fitness to the mean for progeny pools that ranged from $n=2$ to 200 . Progeny pools were generated by randomly selecting from log-normal distributions, and selection was calculated as $s=$ $w_{\max }-w_{\text {mean }}$ for each progeny pool of size $n$. We used these data to estimate the relationship between selection $(s)$ and progeny number $(s=0.0198 \ln (n)+0.0276$; where $n$ is the number of progeny). These estimates indicate that selection increases rapidly for $n<100$ ( $s=0.04$ to 0.12 for $n=2$ to 100 , respectively), and much more slowly when $n>$ 100 (e.g., $s=0.16$ when $n=1000$ ). We note that many plants are capable of producing hundreds of seeds each year, and the large majority of seedlings do not survive to reproduction. We chose to explore a range of progeny numbers from $n=2$ to 200 per generation for each lineage to provide a representative range of selection coefficients. The blue line represents calculated values from log-normal distributions and the black line is the best-fit relationship.

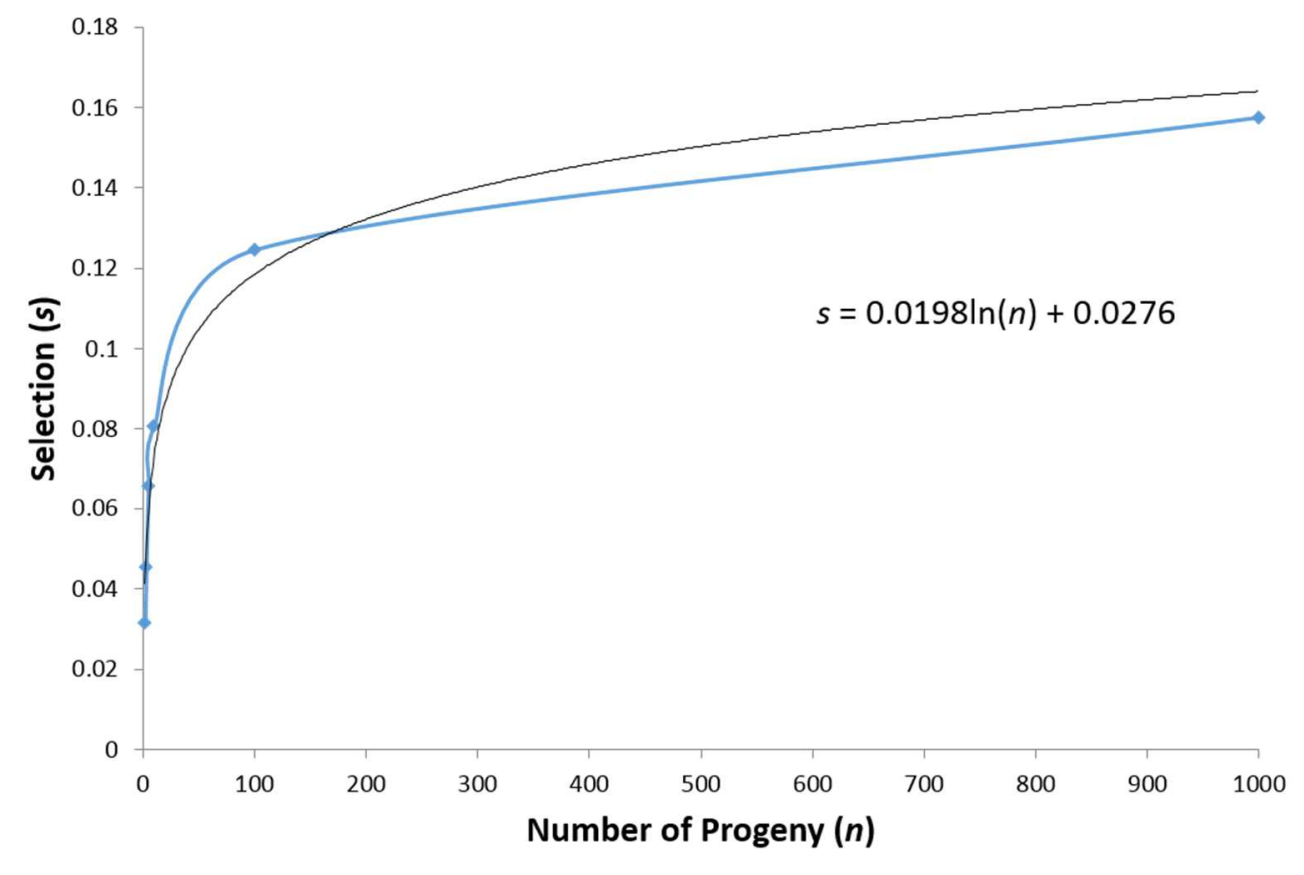


Methods A.A3 Nuclear microsatellite data

Seedlings from each maternal parent were determined to be products of self- or outcross-fertilization using genetic markers (SSRs; Ramakrishnan et al. 2008b; Rosenthal et al. 2008a). Leaf material for genetic analysis was collected from greenhouse plants, dried and stored on silica gels prior to extractions. DNA was extracted after leaf material was flash-frozen in liquid nitrogen and ground in a ball mill (Retcsh M300; Newtown, PA, USA); DNA was purified using DNEasy ${ }^{\mathrm{TM}}$ extraction kits (Qiagen; Valencia, CA, USA ).

Nuclear genomic regions containing repeated motifs of two, three, or four bases were amplified following standardized protocols: $1 \mu \mathrm{L}$ of genomic DNA was amplified by combining $1 \mu \mathrm{L}$ of $2.5 \mu \mathrm{m}$ primer mix with $3.75 \mu \mathrm{L}$ HotStarTaq Master Mix (Qiagen), and 2.25 $\mu \mathrm{L}$ PCR grade water. The primer mixes consisted of a 10:9:1 ratio of forward primer, reverse primer, and fluorophore- labeled reverse primer, respectively. Cycling reactions were conducted either on an MJ Research P-100 (St. Bruno, Quebec, Canada) or an Eppendorf Master Gradient (Hauppauge, NY, USA). Reaction conditions were: $95^{\circ} \mathrm{C}$ for $15 \mathrm{~min}$, then $95^{\circ} \mathrm{C}$ for $30 \mathrm{~s}, 58-60^{\circ} \mathrm{C}$ for $45 \mathrm{~s}$, and $72^{\circ} \mathrm{C}$ for $30 \mathrm{~s}$ for $30-40$ cycles, then $72^{\circ} \mathrm{C}$ for $2 \mathrm{~min}$. Specific cycling times and annealing temperatures varied depending on which primer combinations were used. All primers were developed and optimized at Portland State University (Ramakrishnan et al. 2008b; Rosenthal et al. 2008a) and are deposited in GenBank. To ensure that multiplexing had no effect on the size of the amplified DNA fragment, multiplexed loci were amplified singly for several individuals and compared to multiplex banding profiles. Microsatellite DNA fragment length variation was visualized on an Applied Biosystems 310 automated capillary 
electrophoresis system (Life Technologies; Grand Island, NY, USA) with GeneScan 500 ROX (Life Technologies) as a standard at Portland State University. Allele length variation was scored with Genemapper ${ }^{\mathrm{TM}}$ software (Life Technologies).

A seedling was determined to be the product of self-fertilization if alleles at all eleven loci matched maternal alleles. Samples were only used in the analysis if they displayed non-ambiguous results at all loci. Because offspring possessing alleles that were not unique to the maternal plant could be the product of selfing or outcrossing with an individual having a similar genotype, we calculated the probability of a plant producing an undetectable outcross event, $P(u)_{j}$ (Cruzan et al. 1994). This probability can be found as the product across all loci of the frequency of maternal alleles in the pollen pool:

$$
P(u)_{j}=\prod_{\mathrm{k}=1}^{\mathrm{m}} p_{\mathrm{ik}},
$$

where $p_{\text {ik }}$ is the population frequency of allele $i$ at locus $k$ present in the maternal genotype. 
Figure A.A3 The effects of the number of populations (metapopulation size $\tilde{N}=1$ to 20 populations) on levels of genetic diversity maintained within populations (average Aa and AA) and fixed for all populations (Fixed for aa and AA across all lineages in all populations). Selection is based on $n=100$ progeny each generation for each line. Deleterious mutations occur at a rate of one per genome per generation $(U=1)$ with $k=$ 100 loci. Values are from simulations after stabilization ( $>50$ generations). Error bars were too small for accurate representation.

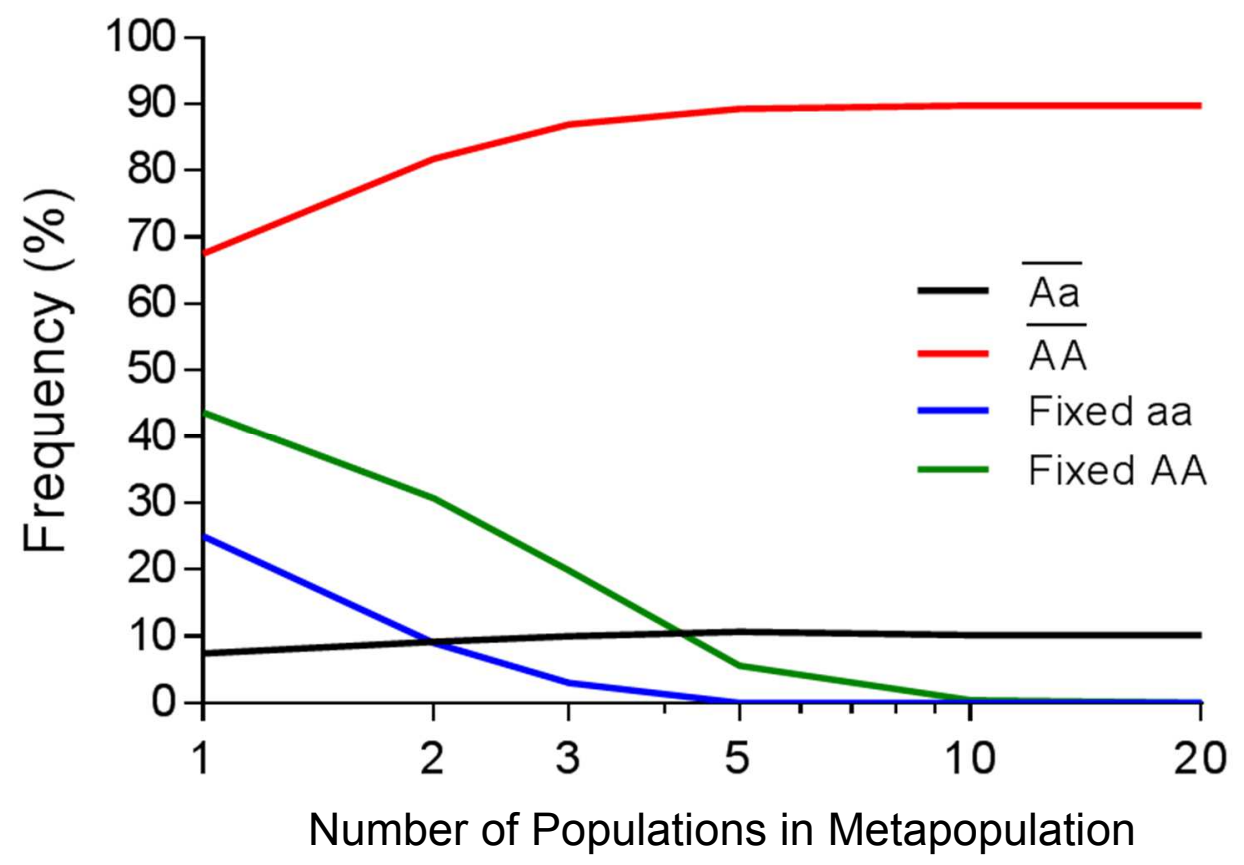




\section{Appendix B}

Supplemental Material for Chapter 3: Ecological mitigation of inbreeding depression in a newly invasive species.

Figure A.B1 Competition Schematic

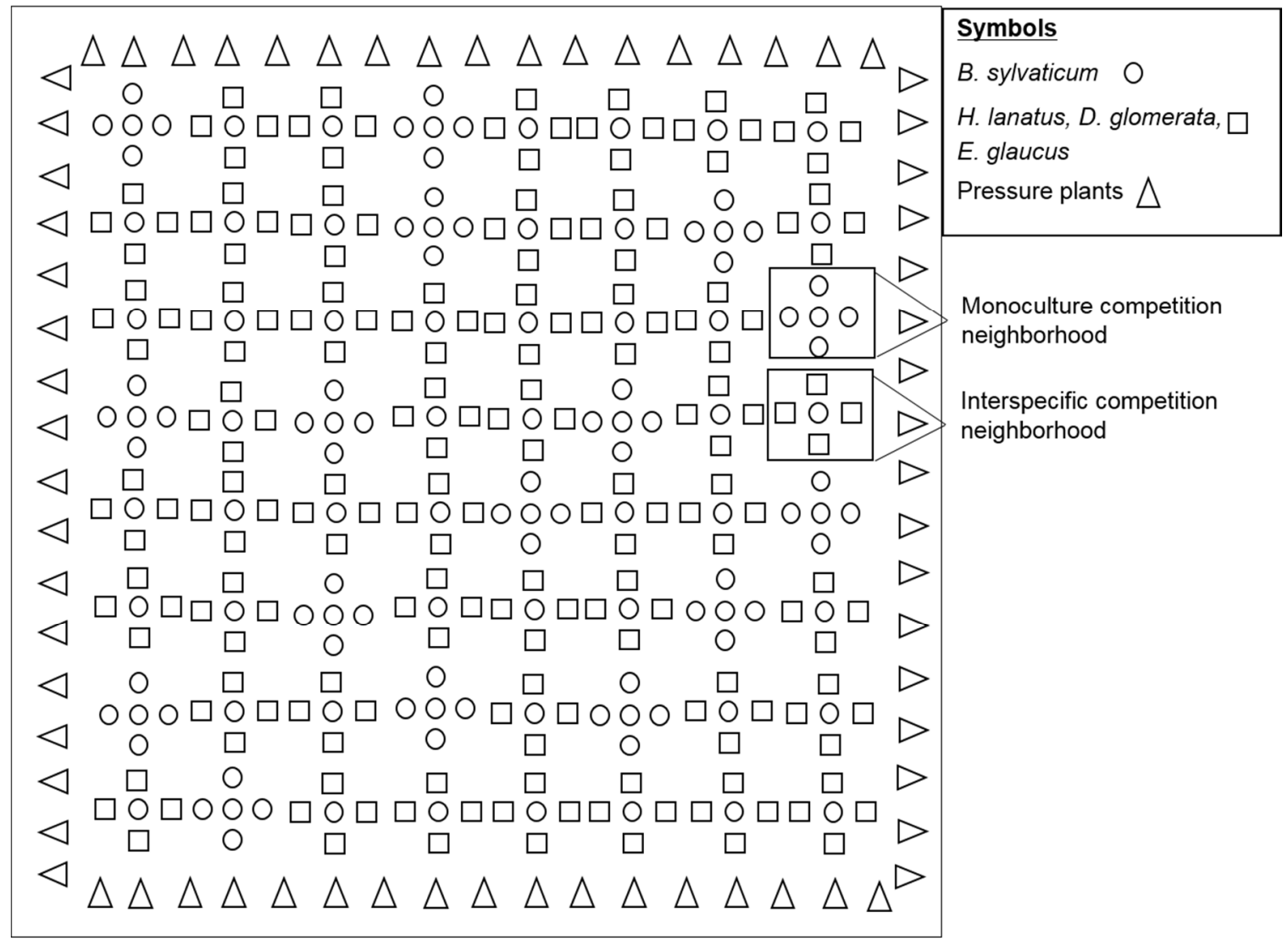

Schematic of competition design within a raised bed in the hoophouse. Six raised beds total were utilized, three of which received nitrogen fertilization. A single individual of Brachypodium sylvaticum was planted at the center of each competition neighborhood, and surrounded at four corners by conspecific individuals of each competitor species (Holcus lanatus, Dactylis glomerata, Elymus glaucus, or Brachypodium sylvaticum).

Focal B. sylvaticum seedlings were spaced $10 \mathrm{~cm}$ apart in the center of each 
neighborhood, with competitor seedlings located at the four corners of each square At the edge of the raised beds "pressure plants" were planted randomly by species to control for competitive effect in edge neighborhoods. 


\section{Appendix B $F_{\text {IS }}$ X Nitrogen Interactions}

The inbreeding coefficient $\left(F_{\text {IS }}\right)$ measures the extent to which consanguineous mating occurs in a population and can signify fitness losses in individuals from populations with homozygote excess (Frankham 1997). $F_{\mathrm{IS}}$ is a function of $N_{\mathrm{e}}$, because in small populations even random mating may be between closely related individuals (Keller and Waller 2002). The inclusion of both $H_{\mathrm{S}}$ and "population" or $F_{\text {IS }}$ and "population" ("population" as a categorical variable) within the same model would confound results, but we are including results from statistical tests using $F_{\text {IS }}$ in this appendix. Results from models substituting $F_{\text {IS }}$ for $H_{\mathrm{S}}$ were equivalent, so these results are not described in the manuscript.

The local inbreeding coefficient $F_{\text {IS }}$ was positive in 10 of the 11 populations surveyed, with an average $F_{\mathrm{IS}}=0.489$, indicating a tendency towards inbreeding in invasive B. sylvaticum (Table B1). Significant differences from zero in $F_{\text {IS values were }}$ found by bootstrapping 1000 times over loci utilizing the R package hierfstat (Goudet 2005). Seven out of 11 total populations displayed $F_{\text {IS }}$ values that deviated from zero (Table B1), and there is a significantly positive relationship between $H_{\mathrm{S}}$ and $F_{\mathrm{IS}}$ as indicated by a Pearson correlation coefficient of $R=0.7042(p=0.02)$.

Incremental seasonal growth was predicted by $F_{\text {Is. Individuals from populations }}$ with low $F_{\text {IS }}$ had increased vegetative growth throughout the first $\left(\mathrm{F}_{1,15}=11.00, \mathrm{p}=\right.$ $0.0010)$ and third experimental seasons $\left(\mathrm{F}_{1,9}=18.21, \mathrm{p}<0.0001\right)$. The Nitrogen $\mathrm{x} F_{\text {IS }}$ interaction influenced final plant size, as nitrogen addition significantly increased the size 
of individuals from high $F_{\text {IS }}$ populations $\left(\mathrm{F}_{1,15}=3.28, \mathrm{p}=0.0004\right.$; Figure B1). Mean final size of individuals sourced from high $F_{\text {IS }}$ populations with nitrogen addition was equal to $132.27 \pm 12.53$ (tiller number $x$ height) compared to a mean final size of $43.53 \pm 3.66$ (tiller number $\mathrm{x}$ height) for plants grown without nitrogen addition (Figure B1). Individuals from low $F_{\text {IS }}$ sources were associated with greater final leaf chlorophyll content $\left(\mathrm{F}_{1,16}=5.89, \mathrm{p}=0.0156\right)$.

Table A.B1: Location information, $H_{S}, H_{O}$, and $F_{I S}$ values for $B$. sylvaticum populations.

\begin{tabular}{lllllll}
\hline Site Code & Latitude & Longitude & $\mathrm{N}$ & $H_{S}$ & $H_{O}$ & $F_{I S}$ \\
\hline C1 & $44^{\circ} 39^{\prime} 35^{\prime \prime}$ & $-124^{\circ} 45^{\prime} 41^{\prime \prime}$ & 24 & 0.26 & 0.017 & $0.936^{*}$ \\
E1 & $43^{\circ} 57^{\prime} 35^{\prime \prime}$ & $-123^{\circ} 15^{\prime} 49^{\prime \prime}$ & 34 & 0.276 & 0.085 & $0.692^{*}$ \\
E4 & $44^{\circ} 0^{\prime} 48^{\prime \prime}$ & $-123^{\circ} 7^{\prime} 30^{\prime \prime}$ & 14 & 0.042 & 0.036 & 0.148 \\
E6 & $43^{\circ} 59^{\prime} 47^{\prime \prime}$ & $-123^{\circ} 12^{\prime} 3^{\prime \prime}$ & 24 & 0.37 & 0.073 & $0.817^{*}$ \\
E7 & $43^{\circ} 58^{\prime} 29^{\prime \prime}$ & $-123^{\circ} 21^{\prime} 13^{\prime \prime}$ & 21 & 0.097 & 0.043 & $0.561^{*}$ \\
E9 & $43^{\circ} 58^{\prime} 26^{\prime \prime}$ & $-123^{\circ} 7^{\prime} 36^{\prime \prime}$ & 24 & 0.392 & 0.176 & $0.544^{*}$ \\
M16 & $44^{\circ} 22^{\prime} 19^{\prime \prime}$ & $-123^{\circ} 22^{\prime} 45^{\prime \prime}$ & 24 & 0.297 & 0.19 & $0.361^{*}$ \\
M3 & $44^{\circ} 23^{\prime} 45^{\prime \prime}$ & $-122^{\circ} 28^{\prime} 13^{\prime \prime}$ & 21 & 0 & 0 & - \\
M5 & $44^{\circ} 24^{\prime} 30^{\prime \prime}$ & $-122^{\circ} 36^{\prime} 22^{\prime \prime}$ & 21 & 0.2 & 0.05 & 0.61 \\
M7 & $44^{\circ} 24^{\prime} 7^{\prime \prime}$ & $-122^{\circ} 39^{\prime} 59^{\prime \prime}$ & 21 & 0.34 & 0.14 & 0.6 \\
S1 & $44^{\circ} 45^{\prime} 14^{\prime \prime}$ & $-123^{\circ} 28^{\prime} 56^{\prime \prime}$ & 29 & 0.063 & 0.086 & $-0.379^{*}$ \\
\hline Asterisks indicate a significant departure from zero $(\mathrm{p}<0.05)$. &
\end{tabular}


Figure A.B2 $(A)$ Nitrogen $\mathrm{x} H_{\mathrm{S}}$ and $(B)$ Nitrogen $\mathrm{x} F_{\mathrm{IS}}$ interaction effects on final size (height $\mathrm{x}$ tiller) of $B$. sylvaticum focal plants. In $(A)$ black bars indicate low $H_{\mathrm{S}}$, white bars indicate high $H_{\mathrm{S}}$. In $(B)$, black bars indicate high $F_{\mathrm{IS}}$, white bars indicate low $H_{\mathrm{S}}$.

Categories were chosen based on natural breaks in the distribution of values: Low $H_{\mathrm{S}}: 0$ to 0.097 , High $H_{\mathrm{S}}: 0.2$ to 0.392 ; Low $F_{\text {IS }}:-0.379$ to 0.361 , High $F_{\text {IS }}: 0.544$ to 0.936 . A low $H_{\mathrm{S}}$ and a high $F_{\text {IS }}$ are associated with a historically small population size $\left(N_{\mathrm{e}}\right)$. Asterisks represent a significant difference $(p<0.05)$ between individuals from populations within respective nitrogen treatments. Error bars represent $\pm \mathrm{SE}$.

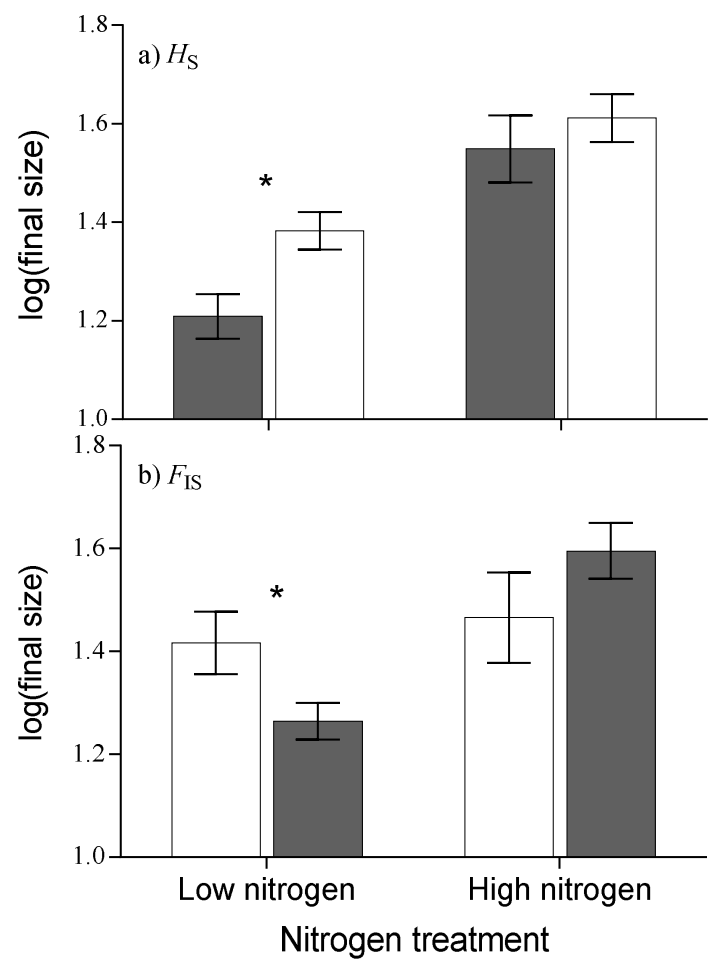

\section{Appendix C}


Supplemental Material for Chapter 4: Selective differentiation during the colonization and establishment of a newly invasive species

Methods A.C1 Microsatellite survey of worldwide populations of B. sylvaticum Nuclear genomic regions containing repeated motifs of two, three, or four bases were amplified following standardized protocols: $1 \mu \mathrm{L}$ of genomic DNA was amplified by combining $1 \mu \mathrm{L}$ of $2.5 \mu \mathrm{m}$ primer mix with $3.75 \mu \mathrm{L}$ HotStarTaq Master Mix, and $2.25 \mu \mathrm{L}$ PCR grade water. The primer mixes consisted of a $10: 9: 1$ ratio of forward primer, reverse primer, and fluorophore-labelled reverse primer, respectively. Cycling reactions were conducted either on an MJ Research P-100 thermal cycler or an Eppendorf Master Gradient. Reaction conditions were: $95^{\circ} \mathrm{C}$ for $15 \mathrm{~min}$, then $95^{\circ} \mathrm{C}$ for $30 \mathrm{~s}, 58-60{ }^{\circ} \mathrm{C}$ for $45 \mathrm{~s}$, and $72{ }^{\circ} \mathrm{C}$ for $30 \mathrm{~s}$ for $30-40$ cycles, then $72{ }^{\circ} \mathrm{C}$ for $2 \mathrm{~min}$. Specific cycling times and annealing temperatures varied depending on which primer com- binations were used. All primers were developed and optimized at Portland State University (Ramakrishnan et al. in press) and are deposited at GenBank (Accession nos EF450748, EF450751, EF450752, EF450754, EF450756, EF450757, EF450759, EF450765). To ensure that multiplexing had no effect on the size of the amplified DNA fragment, multiplexed loci were amplified singly for several individuals and compared to multiplex banding profiles. Microsatellite DNA fragment length variation was visualized on an Applied Biosystems 310 automated capillary electrophoresis system with GeneScan 500 ROX as a standard at Portland State University. Allele length variation was scored with genotyperTM software (Applied Biosystems). Observed $\left(H_{\mathrm{O}}\right)$ heterozygosity was calculated using genalex (Peakall \& Smouse 2006).

Methods A.C2 Climatic comparison across native and invasive populations of B. sylvaticum. 
Climatic data for Oregon, USA populations were obtained from the Prism Group, Oregon State University (http:/www.prismclimate.org), climatic data for native source populations located throughout Europe, the Middle East, and Asia were obtained using WorldClim Version 1(worldclim.org). From the obtained climatic data, we calculated potential evapotranspiration (PET) for each site using Malmstrom's (1969) method as $P E T=40.9 \times e_{s} T_{a}$

where $e_{s} T_{a}$ is the saturation vapor pressure at the mean daily temperature at each site (Malmstrom 1969). We then calculated aridity (A) for each site as $A=P / P E T$

where $P$ is the monthly precipitation and $P E T$ is the monthly potential evapotranspiration (Budyko 1974; Thornthwaite 1948).

Figure A.C1 Comparison of aridity in invasive and native regions of the invasive grass B. sylvaticum.

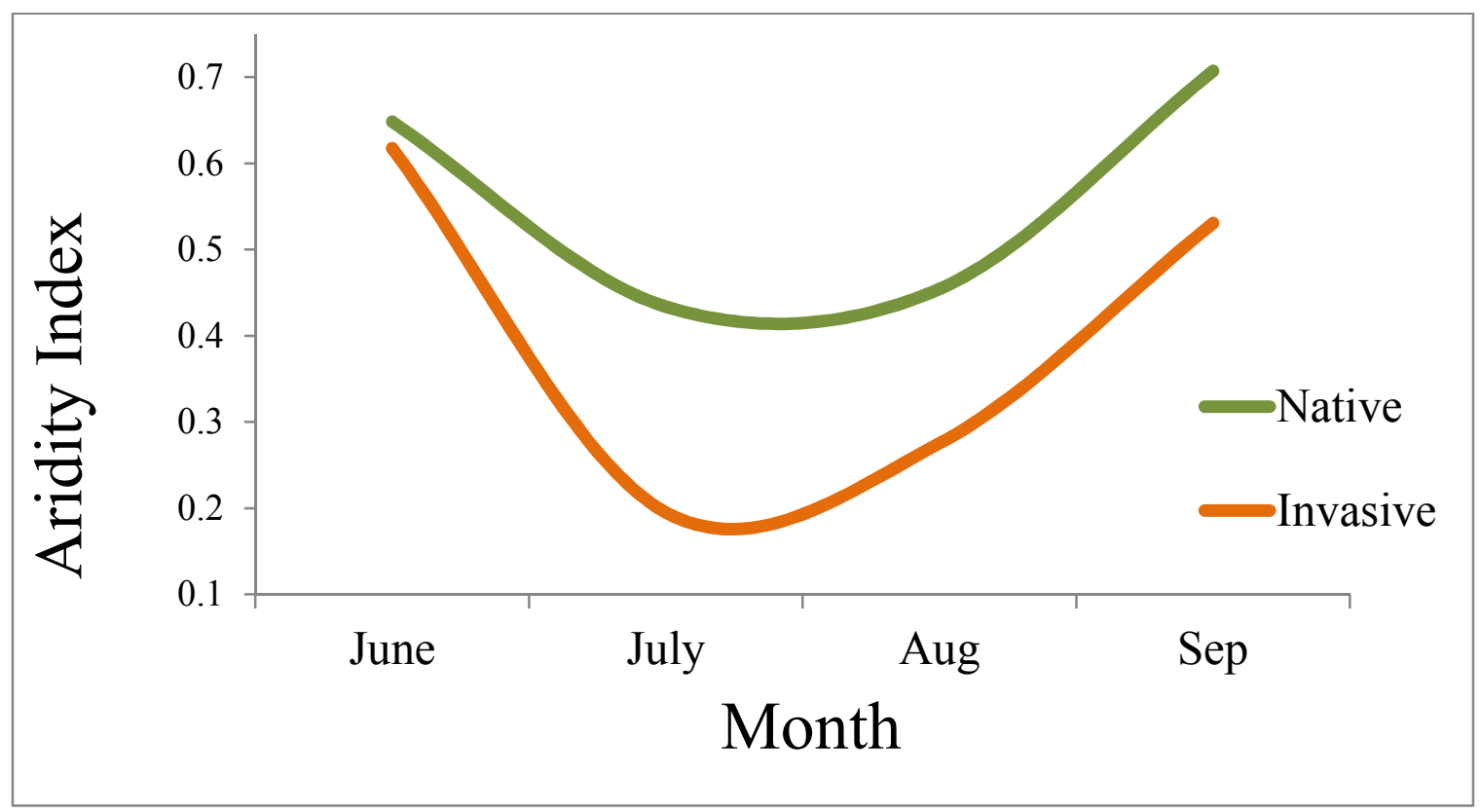

\section{Appendix D}


Supplemental Materials for Chapter 5: Trait divergence, not plasticity, determines the success of a newly invasive plant

Table A.D1 Location information for sampled populations of Brachypodium sylvaticum.

\begin{tabular}{|c|c|c|c|}
\hline \multirow[t]{2}{*}{ Code } & Location & Latitude & Longitude \\
\hline & \multicolumn{3}{|l|}{ NATIVE } \\
\hline ESH & England, Shropshire & $52^{\circ} 37^{\prime} 32^{\prime \prime}$ & $-3^{\circ} 44^{\prime} 14^{\prime \prime}$ \\
\hline ES & England, Surrey & $51^{\circ} 23^{\prime} 24^{\prime \prime}$ & $-1^{\circ} 22^{\prime} 48^{\prime \prime}$ \\
\hline SPIN & Huesca, Spain & $42^{\circ} 8^{\prime} 12^{\prime \prime}$ & $-1^{\circ} 35^{\prime} 24^{\prime \prime}$ \\
\hline TUN & Tunisia & $34^{\circ} 32^{\prime} 29^{\prime \prime}$ & $9^{\circ} 13^{\prime} 20^{\prime \prime}$ \\
\hline Gerh & German Halle & $51^{\circ} 28^{\prime} 12^{\prime \prime}$ & $11^{\circ} 57^{\prime} 36^{\prime \prime}$ \\
\hline Gerl & German Leipzig & $51^{\circ} 20^{\prime} 24^{\prime \prime}$ & $12^{\circ} 22^{\prime} 48^{\prime \prime}$ \\
\hline IC & Italy, Calabria & $39^{\circ} 31^{\prime} 48^{\prime \prime}$ & $16^{\circ} 12^{\prime} 36^{\prime \prime}$ \\
\hline $\mathrm{ICB}$ & Italy, Calabria 2 & $39^{\circ} 32^{\prime} 18^{\prime \prime}$ & $16^{\circ} 12^{\prime} 31^{\prime \prime}$ \\
\hline IP & Italy, Puglia & $40^{\circ} 52^{\prime} 48^{\prime \prime}$ & $16^{\circ} 45^{\prime} 36^{\prime \prime}$ \\
\hline GIK & Greece, Kerkira & $39^{\circ} 31^{\prime} 48^{\prime \prime}$ & $19^{\circ} 55^{\prime} 48^{\prime \prime}$ \\
\hline SES & $\begin{array}{l}\text { Slovakia, East } \\
\text { Slovakia }\end{array}$ & $48^{\circ} 49^{\prime} 41^{\prime \prime}$ & $20^{\circ} 9^{\prime} 4^{\prime \prime}$ \\
\hline SER & Vlakca, Serbia & $44^{\circ} 10^{\prime} 17^{\prime \prime}$ & $20^{\circ} 41^{\prime} 54^{\prime \prime}$ \\
\hline GII & Greece, Ioannina & $29^{\circ} 40^{\prime} 12^{\prime \prime}$ & $20^{\circ} 50^{\prime} 24^{\prime \prime}$ \\
\hline GIP & Greece, Ioannina 2 & $29^{\circ} 40^{\prime} 12^{\prime \prime}$ & $20^{\circ} 50^{\prime} 24^{\prime \prime}$ \\
\hline GTC & Greece, Larrisa & $39^{\circ} 32^{\prime} 47^{\prime \prime}$ & $22^{\circ} 8^{\prime} 11^{\prime \prime}$ \\
\hline GMT & Greece, Thessaloniki & $40^{\circ} 37^{\prime} 48^{\prime \prime}$ & $22^{\circ} 57^{\prime} 36^{\prime \prime}$ \\
\hline UKR & Ukrain, Krym & $44^{\circ} 24^{\prime} 14^{\prime \prime}$ & $33^{\circ} 49^{\prime} 30^{\prime \prime}$ \\
\hline TUR & Turkey, Gerze, Sinop & $41^{\circ} 49^{\prime} 2^{\prime \prime}$ & $35^{\circ} 1^{\prime} 24^{\prime \prime}$ \\
\hline RUS & Russian Federation & $45^{\circ} 0^{\prime} 24^{\prime \prime}$ & $41^{\circ} 58^{\prime} 29^{\prime \prime}$ \\
\hline IRAN & Iran & $35^{\circ} 5^{\prime} 21^{\prime \prime}$ & $52^{\circ} 20^{\prime} 20^{\prime \prime}$ \\
\hline \multirow[t]{2}{*}{ KAZ } & Kazakastan, Alma Ata & $43^{\circ} 16^{\prime} 24^{\prime \prime}$ & $76^{\circ} 55^{\prime} 54^{\prime \prime}$ \\
\hline & \multicolumn{3}{|l|}{ INVASIVE } \\
\hline $\mathrm{C} 1$ & Corvallis & $44^{\circ} 39^{\prime} 35^{\prime \prime}$ & $-124^{\circ} 45^{\prime} 41^{\prime \prime}$ \\
\hline C6 & Hwy22 & $44^{\circ} 45^{\prime} 19^{\prime \prime}$ & $-123^{\circ} 36^{\prime} 42^{\prime \prime}$ \\
\hline $\mathrm{C} 10$ & Corvallis & $44^{\circ} 23^{\prime} 29^{\prime \prime}$ & $-124^{\circ} 38^{\prime} 4^{\prime \prime}$ \\
\hline E1 & Eugene & $43^{\circ} 57^{\prime} 35^{\prime \prime}$ & $-123^{\circ} 15^{\prime} 49^{\prime \prime}$ \\
\hline E4 & Eugene & $44^{\circ} 0^{\prime} 48^{\prime \prime}$ & $-123^{\circ} 7^{\prime} 30^{\prime \prime}$ \\
\hline E6 & Eugene & $43^{\circ} 59^{\prime} 47^{\prime \prime}$ & $-123^{\circ} 12^{\prime} 3^{\prime \prime}$ \\
\hline E7 & Eugene & $43^{\circ} 58^{\prime} 29^{\prime \prime}$ & $-123^{\circ} 21^{\prime} 13^{\prime \prime}$ \\
\hline E9 & Eugene & $43^{\circ} 58^{\prime} 26^{\prime \prime}$ & $-123^{\circ} 7^{\prime} 36^{\prime \prime}$ \\
\hline M1 & Fish_Ck_Cmpgrnd & $44^{\circ} 23^{\prime} 54^{\prime \prime}$ & $-123^{\circ} 39^{\prime} 18^{\prime \prime}$ \\
\hline M16 & Cedar \& Wiley & $44^{\circ} 22^{\prime} 19^{\prime \prime}$ & $-123^{\circ} 22^{\prime} 45^{\prime \prime}$ \\
\hline S1 & Fisherman's Bend & $44^{\circ} 45^{\prime} 14^{\prime \prime}$ & $-123^{\circ} 28^{\prime} 56^{\prime \prime}$ \\
\hline
\end{tabular}


Figure A.D1 Probabilities of genetic contribution from multilocus genotypes.

Probabilities are used as weights in statistical analysis.

Detailed methods of the microsatellite survey performed for determination of genetic weights of contribution are detailed in Marchini and Cruzan (unpublished).

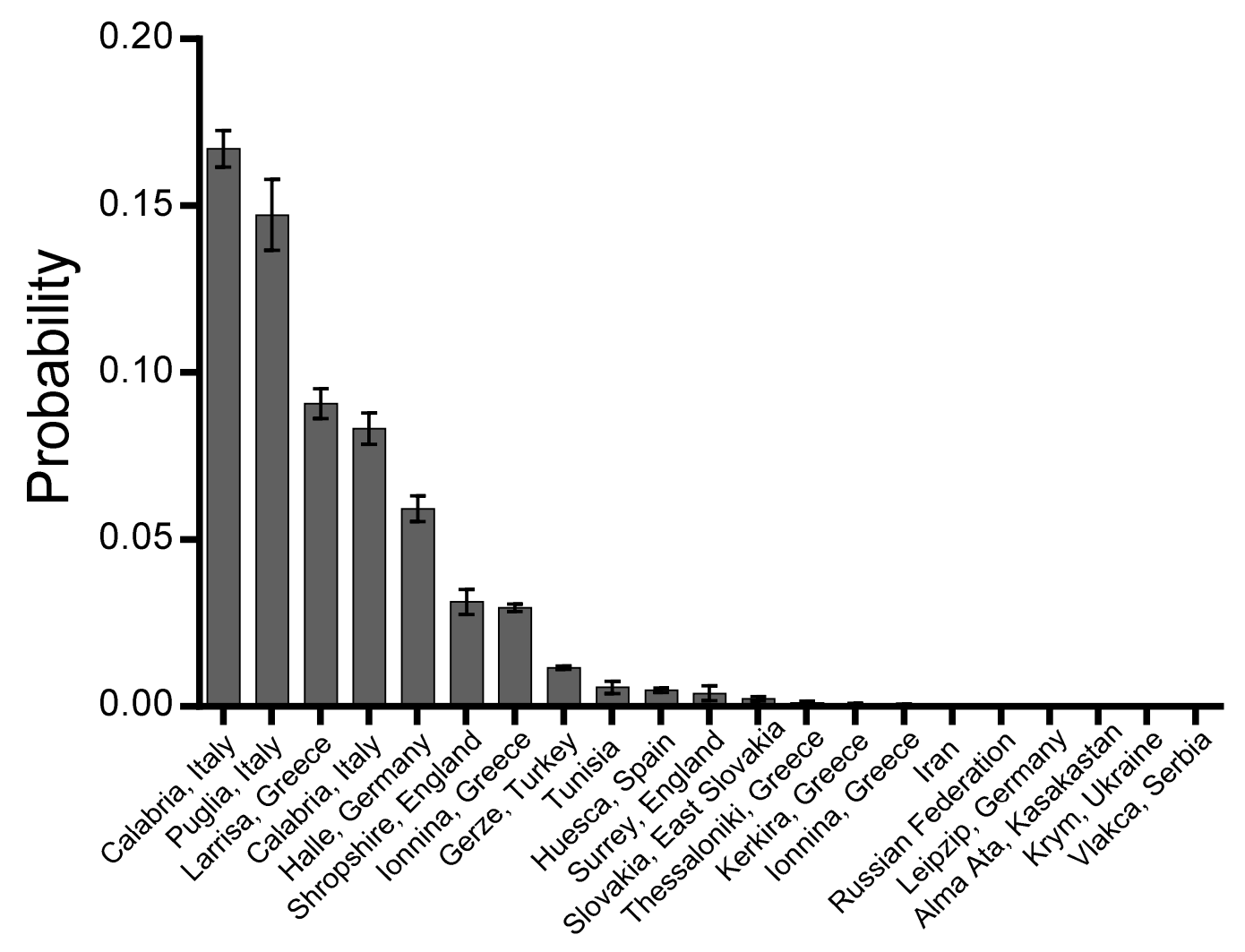

